\title{
Thermodynamic and Kinetic Considerations in the Copolymerization of Ethylene and Carbon Dioxide
}

\author{
Craig J. Price, B. Jesse E. Reich, and Stephen A. Miller*
}

Department of Chemistry, Texas A\&M University

College Station, Texas 77843-3255

\section{Supporting Information}

Supporting Information Available: Theoretical and synthetic details, DFT optimized structures, as well as additional characterization data $\left({ }^{13} \mathrm{C} N \mathrm{NMR}, \mathrm{GC}-\mathrm{MS}\right)$ for the formed oligomers and polymers.

\section{Table of Contents}

General Considerations and Instrumentation

Precatalyst Synthesis

${ }^{13} \mathrm{C}$ NMR Spectra.

GC-MS Spectra

Computational Methods

DFT Structures and Coordinates

References

$$
\begin{aligned}
& \left.\mathrm{CO}_{2}+\mathrm{?} \stackrel{?}{\longrightarrow} \mathrm{H}_{\mathrm{O}}^{\mathrm{O}}\right][
\end{aligned}
$$

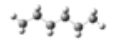

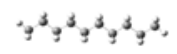

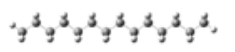

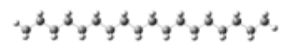

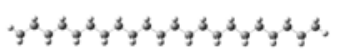

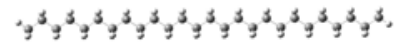

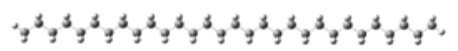

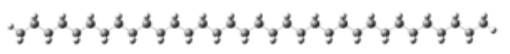

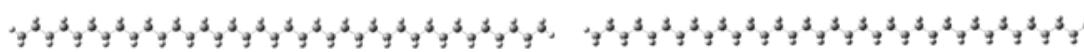

$$
\begin{aligned}
& a_{2} a_{2}
\end{aligned}
$$

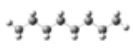

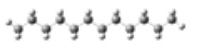

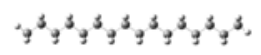

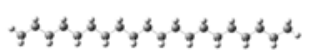

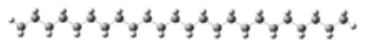

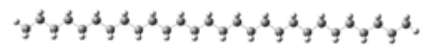

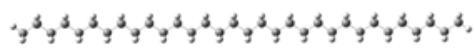

$$
\begin{aligned}
& \mathrm{CO}_{2} \text {. }
\end{aligned}
$$

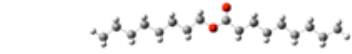

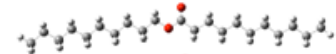

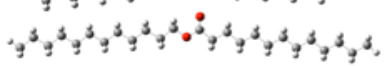

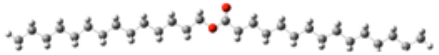

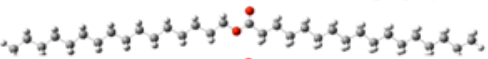

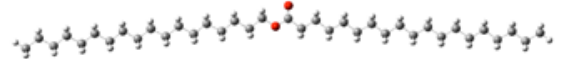


General Considerations and Instrumentation. All air-sensitive procedures were performed under a purified nitrogen atmosphere in a glove box or by using standard Schlenk line and vacuum line techniques. 2,6-Diisopropyl aniline (Acros, $92 \%$ ) was distilled at $145^{\circ} \mathrm{C}$ under dynamic vacuum before use. Methylaluminoxane (MAO) (Albemarle, $30 \%$ in toluene) was concentrated to dryness and used as a solid. Ethanol was distilled from magnesium sulfate into an oven-dried Straus flask and stored until use. Tetrahydrofuran was distilled from sodium/benzophenone under a nitrogen atmosphere and stored in an oven-dried Straus flask until use. All other chemicals and solvents were used as received.

Precatalyst Synthesis. Precatalysts 1 - 5 and the ligand for precatalyst $\mathbf{6}$ were prepared according to the following modified literature procedures.
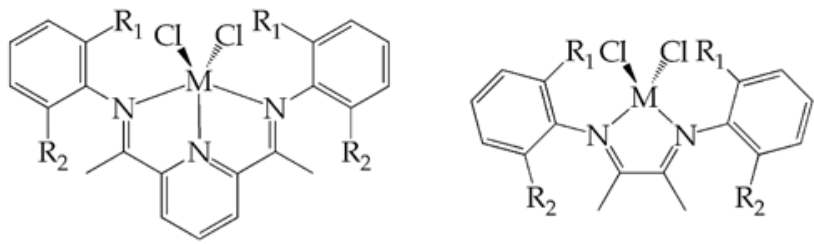

$$
\begin{aligned}
& \text { 1: } M=F e, R_{1}=R_{2}={ }^{i} \operatorname{Pr} \\
& \text { 2: } M=F e, R_{1}=R_{2}=M e \\
& \text { 3: } M=C o, R_{1}={ }^{i} P r, R_{2}=H \\
& \text { 4: } M=F e, R_{1}=M e, R_{2}=H
\end{aligned}
$$

5: $\mathrm{M}=\mathrm{Ni}, \mathrm{R}_{1}=\mathrm{R}_{2}={ }^{i} \mathrm{Pr}$

6: $\mathrm{M}=\mathrm{Cu}, \mathrm{R}_{1}={ }^{i} \mathrm{Pr}, \mathrm{R}_{2}=\mathrm{H}$
Synthesis of $N, N^{\prime}$-bis-(2,6-diisopropylaniline) diacetylpyridine [204203-14-5] (CJP-I-48). In a $50 \mathrm{~mL}$ round bottom flask, $2.00 \mathrm{~g}(12.25 \mathrm{mmol})$ of 2,6-

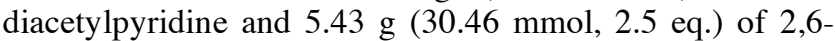
diisopropylaniline were dissolved in $25 \mathrm{~mL}$ of dry ethanol. Five drops of glacial acetic acid were added. The mixture refluxed for 18 hours in an oil bath. The heat was turned off and the mixture was allowed to slowly cool to room temperature in the oil bath. The mixture was cooled in an ice bath for 30 minutes and then filtered to yield $3.103 \mathrm{~g}$ $(52.6 \%)$ of a yellow powder, which was triturated in ethyl acetate. MS (ESI) $m / z 482.3(\mathrm{M}+\mathrm{H})^{+}$

Synthesis of 1 [204203-10-1] or [308359-85-5] (CJPI-49). ${ }^{1}$ Inside the glove box, $2.00 \mathrm{~g}(4.15 \mathrm{mmol})$ of $N, N^{\prime}-$ bis-(2,6-diisopropylaniline) diacetylpyridine and $0.52 \mathrm{~g}$ (4.15 mmol) of $\mathrm{FeCl}_{2} \cdot 4 \mathrm{H}_{2} \mathrm{O}$ were combined in a $200 \mathrm{~mL}$ round bottom flask and a swivel frit apparatus was attached. The frit was evacuated and $75 \mathrm{~mL}$ of dry ethanol were condensed in. The frit was back-filled with nitrogen and the blue solution was refluxed for 30 minutes. The frit was flipped and all soluble material was washed to the lower collection flask. The solution was condensed to approximately half of its original volume and placed in an ice-water bath for 30 minutes. The frit was again flipped to collect $0.658 \mathrm{~g}(26.0 \%)$ of a dark blue crystalline solid. The filtrate was concentrated to dryness and redissolved in dichloromethane. The second crop was purified by the same recrystallization method to yield $0.744 \mathrm{~g}(29.5 \%)$. The total yield of first and second crops is $1.402 \mathrm{~g} \mathrm{(55.5}$ $\%)$. Blue needle-like crystals suitable for X-ray diffraction can be grown by vapor diffusion of hexanes into a saturated dichloromethane solution. The molecule cocrystallizes with 0.5 molecules of $\mathrm{H}_{2} \mathrm{O}$. The $\mathrm{X}$-ray data, shown below, are identical to that previously reported. ${ }^{2}$

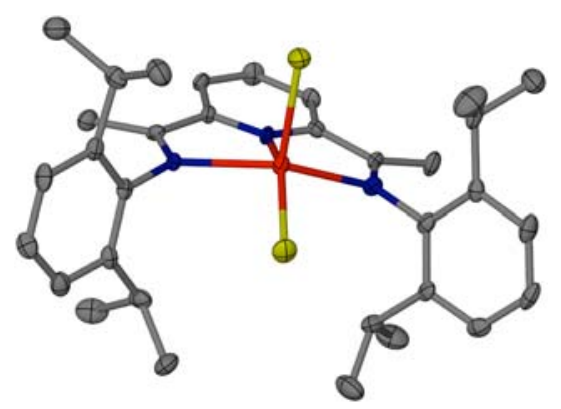

Crystal data: triclinic, $P-1, a=8.6390(10) \AA, \quad b=$ 9.8363(10) $\AA, c=20.714(2) \AA, \alpha=83.513(6)^{\circ}, \beta=$ 88.300(7) ${ }^{\circ}, \gamma=65.758(6)^{\circ}, V=1594.5(3) \AA^{3}, Z=2, T=$ $110(2) \mathrm{K}, \mathrm{R}_{1}\left(\right.$ on $\left.\mathrm{F}_{0}\right)=0.0500, \mathrm{wR}_{2}\left(\right.$ on $\left.\mathrm{F}_{0}{ }^{2}\right)=0.1195, \mathrm{GOF}$ $=1.012$ for 366 parameters and 4244 unique data.

Synthesis of $N, N^{\prime}$-bis-(2,6-dimethylaniline) diacetylpyridine [204203-16-7] (BJR-II-14). A $50 \mathrm{~mL}$ round bottom flask was charged with 2,6-diacetylpyridine (3.00 g, $18.39 \mathrm{mmol}), 2$,6-dimethylaniline, and $30 \mathrm{~mL}$ of ethanol. When the mixture was perceived to be homogeneous, 4 drops of glacial acetic acid were added. The mixture was stirred overnight. The solution was concentrated to $15 \mathrm{~mL}$ and the product was isolated by filtration and washed with $15 \mathrm{~mL}$ of cold ethanol. The product was recrystallized in ethanol and dried in vacuo to yield $4.34 \mathrm{~g}(64.0 \%)$ of yellow crystals.

Synthesis of 2 [207129-93-9] (BJR-II-18). ${ }^{1}$ In the glove box a $100 \mathrm{~mL}$ pear shaped flask was charged with $\mathrm{N}, \mathrm{N}$-bis-(2,6-dimethylaniline) diacetylpyridine $(3.70 \mathrm{~g}, 10$ mmol) and $\mathrm{FeCl}_{2}(1.90 \mathrm{~g}, 15 \mathrm{mmol})$. The flask was attached to a swivel frit and connected to a high vacuum line and evacuated. $20 \mathrm{~mL}$ of dry ethanol were vacuum transferred into the vessel. The frit was opened to $\mathrm{N}_{2}$ and the solution was heated at reflux for 15 minutes. The frit was allowed to cool to room temperature and the frit was flipped. The frit was evacuated and refluxing ethanol was used to extract excess $\mathrm{FeCl}_{2}$ from the reaction solids. After $10-15 \mathrm{~mL}$ of ethanol had washed the material, solvent was removed by high vacuum and the purple powder was dried in vacuo to yield $4.74 \mathrm{~g}(96.0 \%)$.

Synthesis of $N, N$ '-bis-(2-isopropylaniline) diacetylpyridine [210537-34-1] (BJR-II-75). 2,6diacetyl pyridine $(3.00 \mathrm{~g}, \quad 18.4 \mathrm{mmol})$ and 2isopropylaniline $(6.85 \mathrm{~g}, 45.9 \mathrm{mmol})$ were dissolved in 200 $\mathrm{mL}$ of toluene. Para-toluenesulfonic acid monohydrate $(0.10 \mathrm{~g})$ was added. A Dean-Stark trap was attached to the flask and the reaction was heated to reflux. After 16 hours $0.8 \mathrm{~mL}$ of water had come over and the heat source was removed. The solution was poured into a separatory funnel and the organic layer was rinsed with $100 \mathrm{~mL}$ of a dilute aqueous sodium bicarbonate solution $(3 \mathrm{~g} / 100 \mathrm{~mL})$. The aqueous layer was rinsed with diethyl ether $(2 \times 100 \mathrm{~mL})$ and all organics were combined and dried in vacuo. The yellow powder was triturated in refluxing ethanol, cooled to RT, and filtered to yield $6.44 \mathrm{~g}(88.0 \%)$ of the desired product.

Synthesis of 3 [590367-46-7] (BJR-II-83). ${ }^{3}$ Inside the glove box $N, N^{\prime}$-bis-(2-isopropylaniline) diacetylpyridine $(3.10 \mathrm{~g}, 7.8 \mathrm{mmol})$ and $\mathrm{CoCl}_{2}(1.00 \mathrm{~g}, 7.7 \mathrm{mmol})$ were combined in a flask and this was attached to a swivel frit. 
The frit was brought into air and evacuated on a Schlenk line. THF $(40 \mathrm{~mL})$ was vacuum transferred into the flask at liquid nitrogen temperatures and warmed to room temperature. The solution stirred for 16 hours at which time the THF was removed in vacuo and diethyl ether (40 $\mathrm{mL}$ ) was vacuum transferred into the flask. The frit was flipped and gentle reflux of ether allowed for the extraction of the product. The solution was concentrated and the frit flipped again. The powder was collected on the frit and a positive pressure of nitrogen isolated the powder on the frit. The powder was dried in vacuo to yield $3.66 \mathrm{~g}(90.2 \%)$ of the cobalt complex.

$$
\text { Synthesis of } \quad N, N^{\prime} \text {-bis-(2-methylaniline) }
$$

diacetylpyridine [210537-32-9] (BJR-II-74). 2,6diacetyl pyridine $(3.00 \mathrm{~g}, 18.4 \mathrm{mmol})$ and $o$-toluidine $(4.93$ $\mathrm{g}, 45.9 \mathrm{mmol})$ were dissolved in $200 \mathrm{~mL}$ of toluene. Paratoluenesulfonic acid monohydrate $(0.10 \mathrm{~g})$ was added. A Dean-Stark trap was attached to the flask and the reaction was heated to reflux. After 16 hours $0.8 \mathrm{~mL}$ of water had come over and the heat source was removed. The solution was poured into a separatory funnel and the organic layer was rinsed with $100 \mathrm{~mL}$ of a dilute aqueous sodium bicarbonate solution $(3 \mathrm{~g} / 100 \mathrm{~mL})$. The aqueous layer was rinsed with diethyl ether $(2 \times 100 \mathrm{~mL})$ and all organics were combined and dried in vacuo. The yellow powder was recrystallized from hot ethanol and isolated by filtration to yield $3.41 \mathrm{~g}(54.3 \%)$ of crystalline product.

Synthesis of 4 [210537-35-2] (BJR-II-76). ${ }^{4}$ Inside the glove box $N, N$ '-bis-(2-methylaniline) diacetylpyridine $(2.83 \mathrm{~g}, 8.29 \mathrm{mmol})$ and $\mathrm{FeCl}_{2}(1.00 \mathrm{~g}, 7.89 \mathrm{mmol})$ were combined in a flask and this was attached to a swivel frit. The frit was brought into air and evacuated on a Schlenk line. THF $(40 \mathrm{~mL})$ was vacuum transferred into the flask at liquid nitrogen temperatures and warmed to room temperature. The solution stirred for 16 hours at which time the THF was removed in vacuo and hexanes $(40 \mathrm{~mL})$ were vacuum transferred into the flask. The frit was flipped and then gently refluxed to extract the product. Hexanes were removed in vacuo and diethyl ether $(40 \mathrm{~mL})$ was vacuum transferred into the flask. Gentle reflux of ether allowed for the full extraction of the product. Ether was removed and hexanes were again added and the solution concentrated. The frit was flipped again and the powder was collected on the frit. The powder was dried in vacuo to yield $2.94 \mathrm{~g}(79.6 \%)$ of the iron complex.

Synthesis of $N, N^{\prime}$-(butane-2,3-diylidene)-bis-(2,6diisopropylaniline) [74663-77-7] (AKJ-I-97). 2,3- butanedione (12.93 g, $0.16 \mathrm{~mol}$ ) and 2,6-diisopropylaniline $(53.19 \mathrm{~g}, 0.30 \mathrm{~mol})$ were combined in methanol and allowed to shake for 36 hours. The solution was heated until all the material was in solution and then cooled to precipitate a crystalline solid, which was collected by filtration.

Synthesis of 5 [200879-37-4] (BJR-II-51). ${ }^{5}$ In the glove box a $100 \mathrm{~mL}$ pear shaped flask was charged with $\mathrm{NiCl}_{2}(1.94 \mathrm{~g}, 15 \mathrm{mmol})$ and $N, N^{\prime}$-(butane-2,3-diylidene)bis-(2,6-diisopropylaniline) (4.05 g, $10 \mathrm{mmol})$. The flask was attached to a swivel frit and the frit was evacuated on the line. $40-50 \mathrm{~mL}$ of dry ethanol were vacuum transferred into the flask. The flask was exposed to the nitrogen line and the reaction was heated to reflux for 25 minutes. Heat was removed and after one hour, the frit was flipped. By refluxing the solvent, all of the material was extracted through the frit. The volume was concentrated under high vacuum and the frit was flipped. The solid was isolated on the frit and washed with ethanol. Solvent was removed under high vacuum and the product was dried to yield 1.60 g $(29.9 \%)$.

Synthesis of $N, N^{\prime}$-(butane-2,3-diylidene)-bis-(2isopropylaniline) $\quad[\mathbf{4 9 6 7 3 - 3 3 - 8}] \quad$ (BJR-II-73). ${ }^{6}$ 2,3butanedione $(4.30 \mathrm{~g}, 50 \mathrm{mmol})$ and 2-isopropylaniline (13.52 g, $100 \mathrm{mmol})$ were combined in methanol and allowed to shake for 36 hours. The solution was heated until all the material was in solution and then precipitated out to yield $4.77 \mathrm{~g}$ in the first crop, $1.62 \mathrm{~g}$ in the second, $3.18 \mathrm{~g}$ in the third, and $1.47 \mathrm{~g}$ in the fourth for an overall yield of $11.04 \mathrm{~g}(68.9 \%)$.

Synthesis of 6 (BJR-II-79). In air $N, N^{\prime}$-(butane-2,3diylidene)-bis-(2-isopropylaniline) (2.63 g, $8.2 \mathrm{mmol}$ ) was placed in a pear shaped flask and this was attached to a swivel frit. The frit was evacuated and taken into the glove box where $\mathrm{CuCl}_{2}(1.00 \mathrm{~g}, 7.4 \mathrm{mmol})$ was added. The frit was brought onto the line and evacuated. $50 \mathrm{~mL}$ of THF were vacuum transferred into the flask and the reaction was allowed to stir at room temperature for 24 hours. THF was removed in vacuo and diethyl ether was vacuum transferred into the flask. The frit was flipped and gentle reflux allowed for extraction of the product. Solvent was removed in vacuo and hexanes $(50 \mathrm{~mL})$ were vacuum transferred into the flask and gentle reflux further extracted the product. The hexane solution was concentrated and the solid was collected and washed on the frit. In vacuo drying provided $2.79 \mathrm{~g}(82.9 \%)$ of the product. 


\section{${ }^{13}$ C NMR Spectra}

Polymer produced with $\mathbf{1}$ /MAO (Table 5, entry 1), showing the absence of carbonyl peaks:

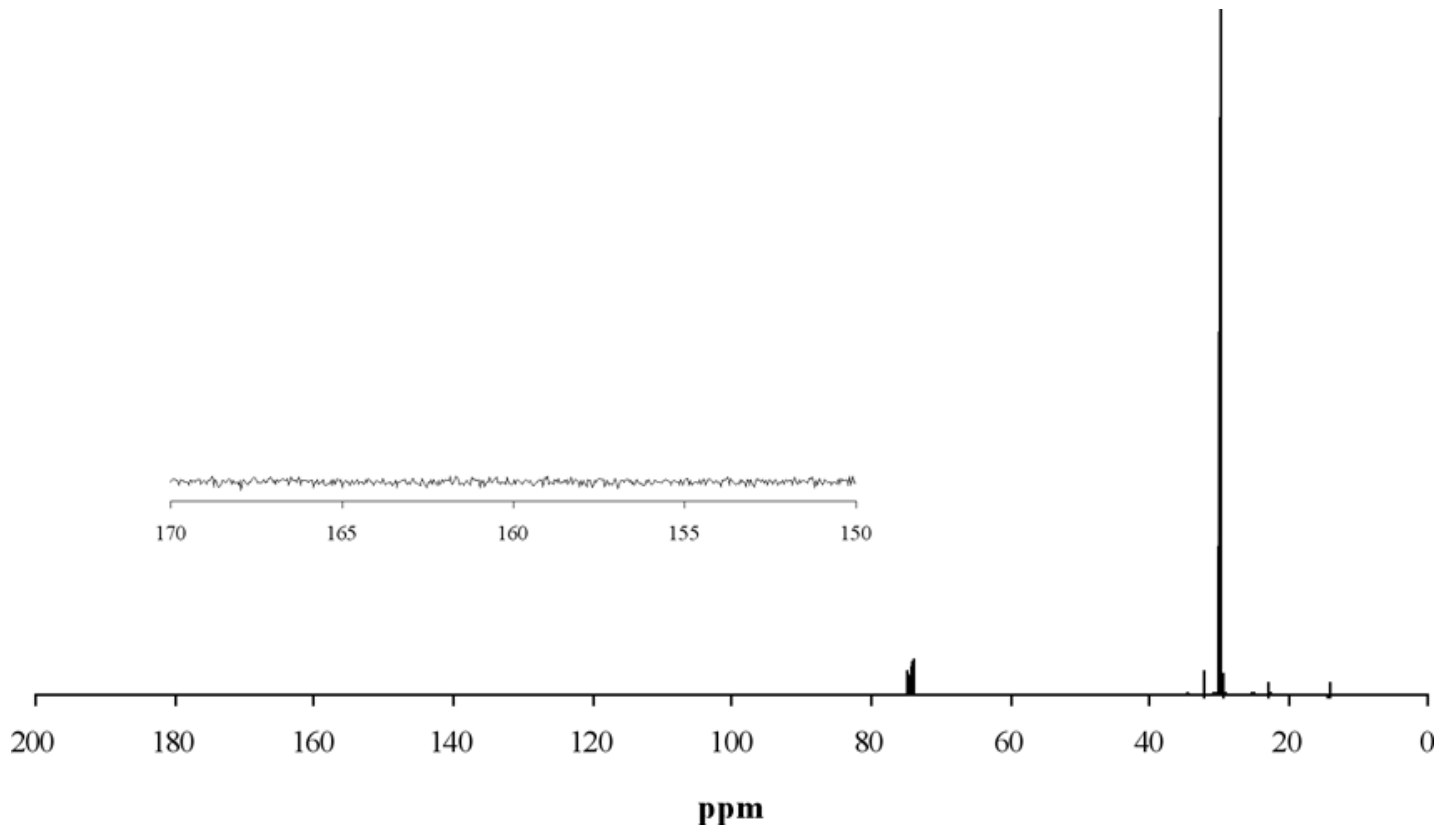

Polymer produced with $\mathbf{1}$ /MAO (Table 5, entry 2), showing the absence of carbonyl peaks:

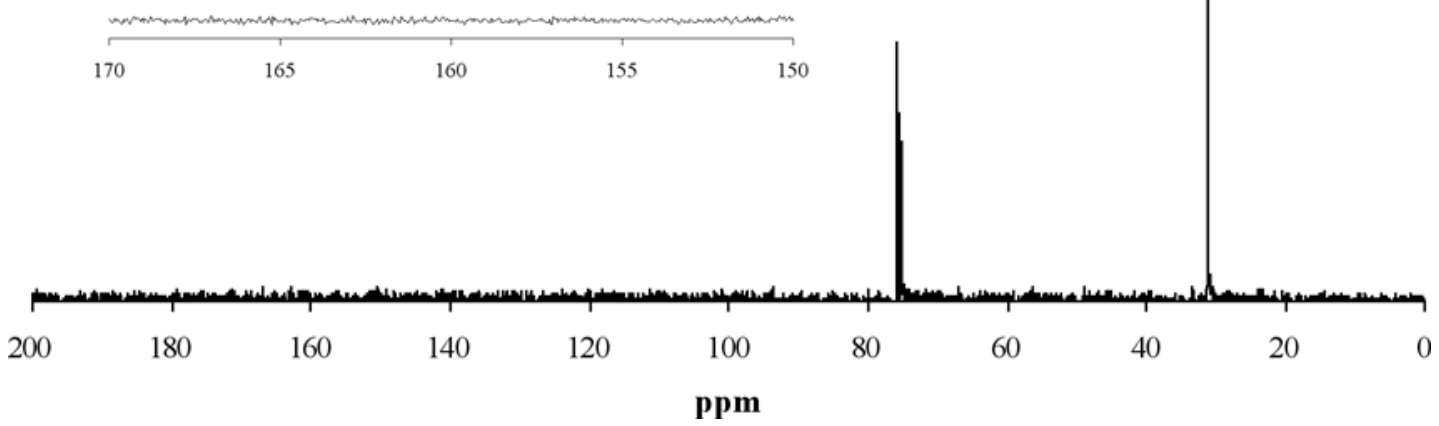


Polymer produced by the homopolymerization of ethylene with 1/MAO (Table 5, entry 3) showing the necessary absence of carbonyl peaks:

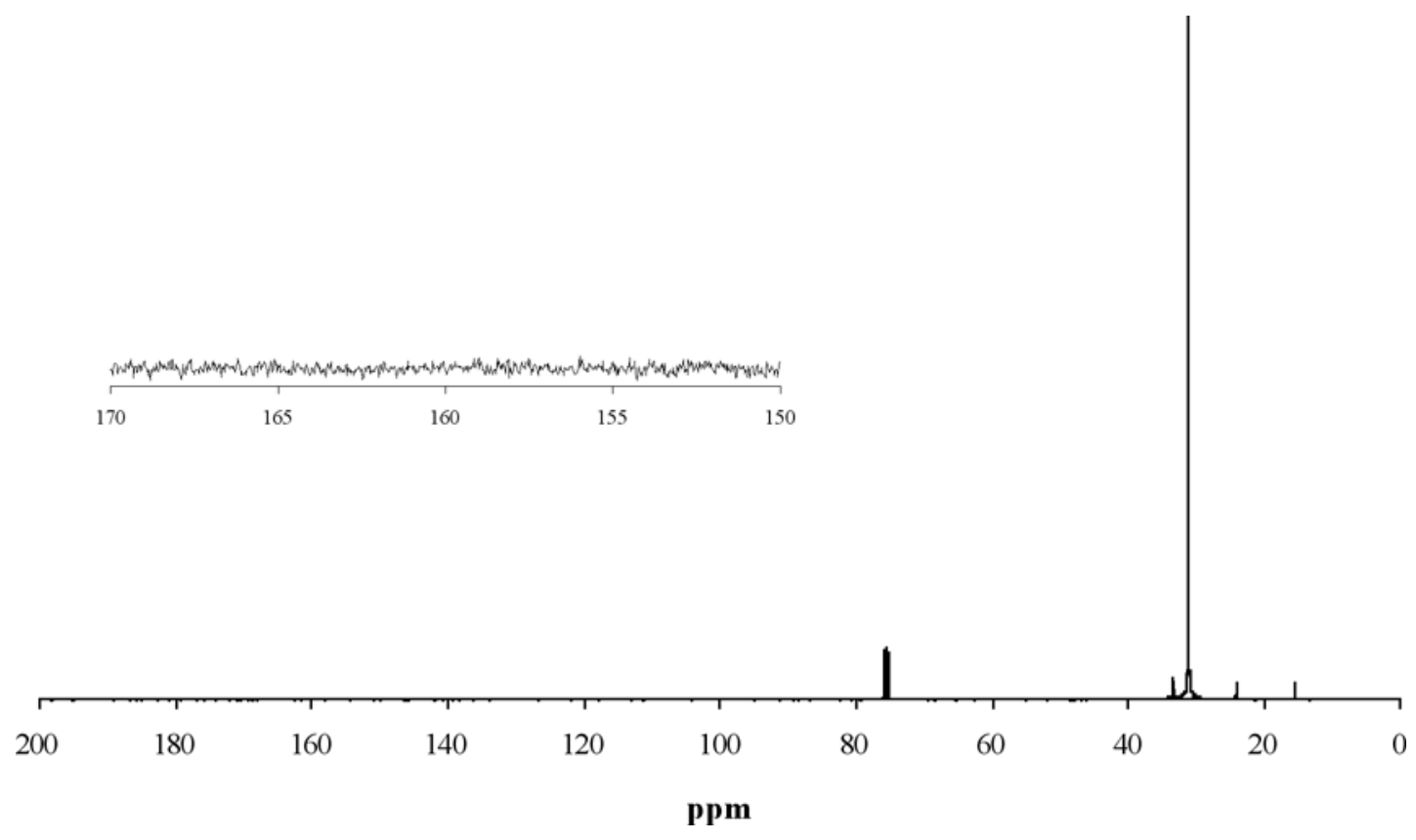

Polymer produced with 2/MAO (Table 5, entry 4), showing the absence of carbonyl peaks:

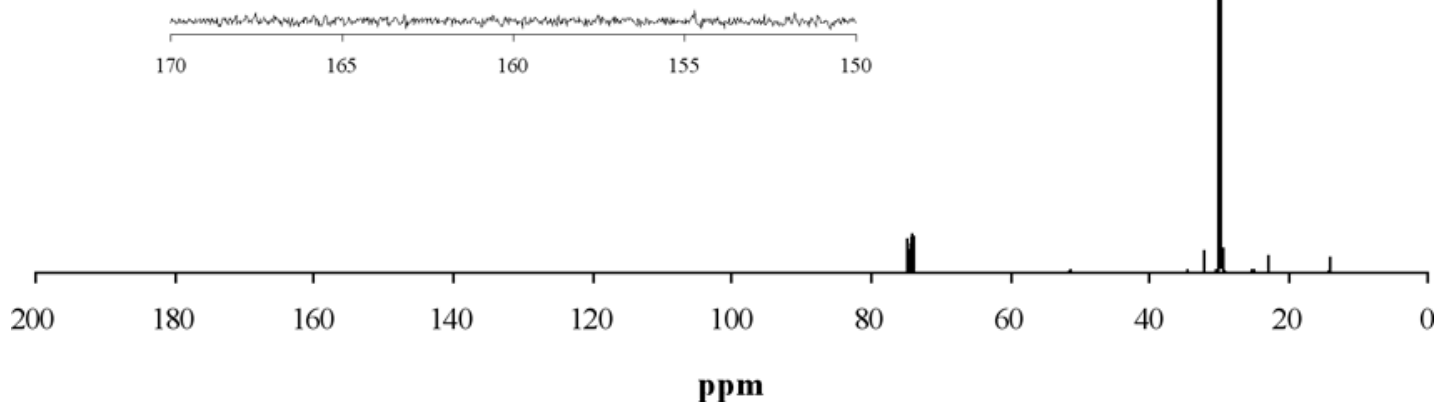


Polymer produced with 2/MAO (Table 5, entry 5), showing the absence of carbonyl peaks:

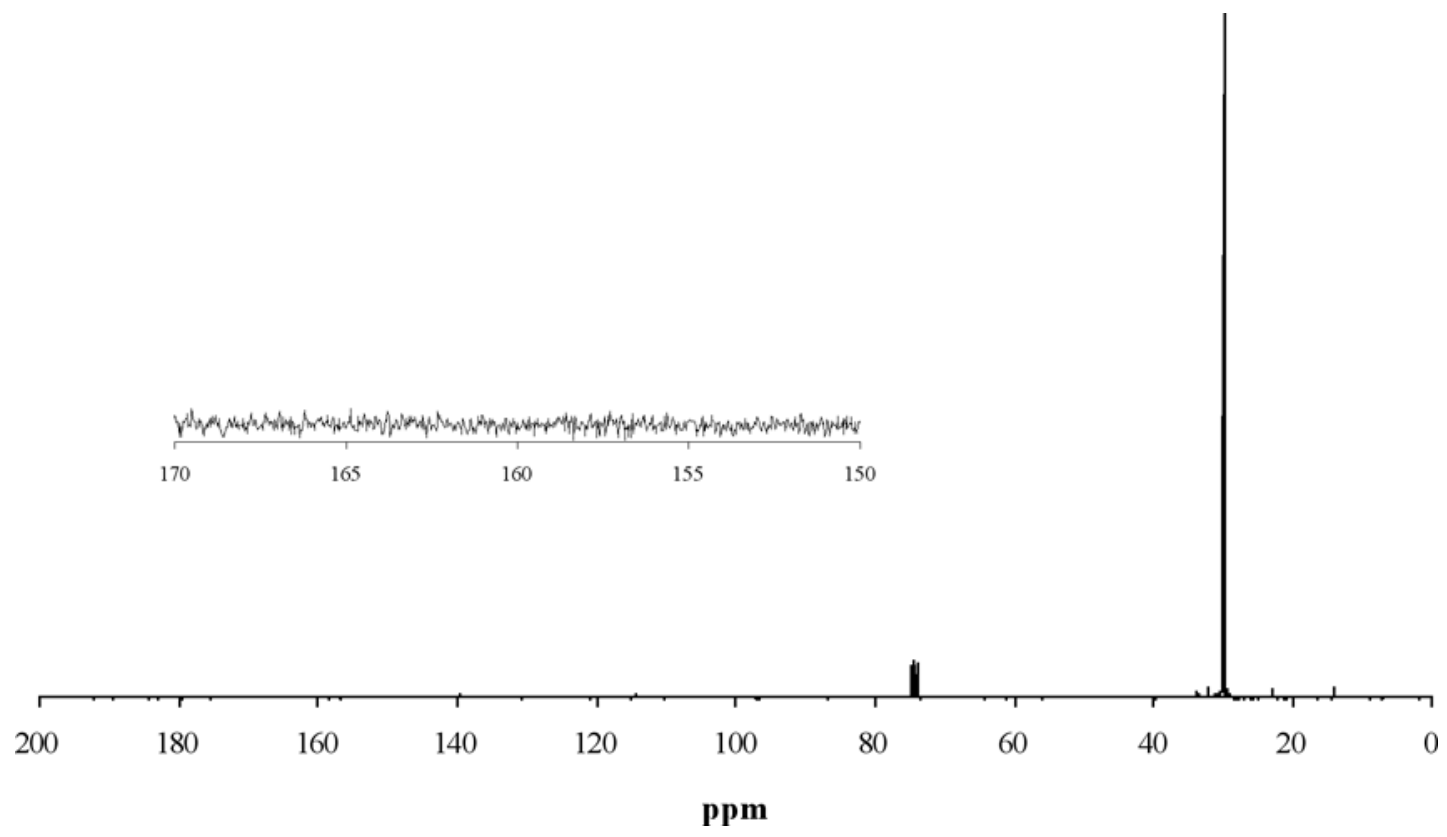

Polymer produced with 2/MAO (Table 5, entry 6), showing the absence of carbonyl peaks:

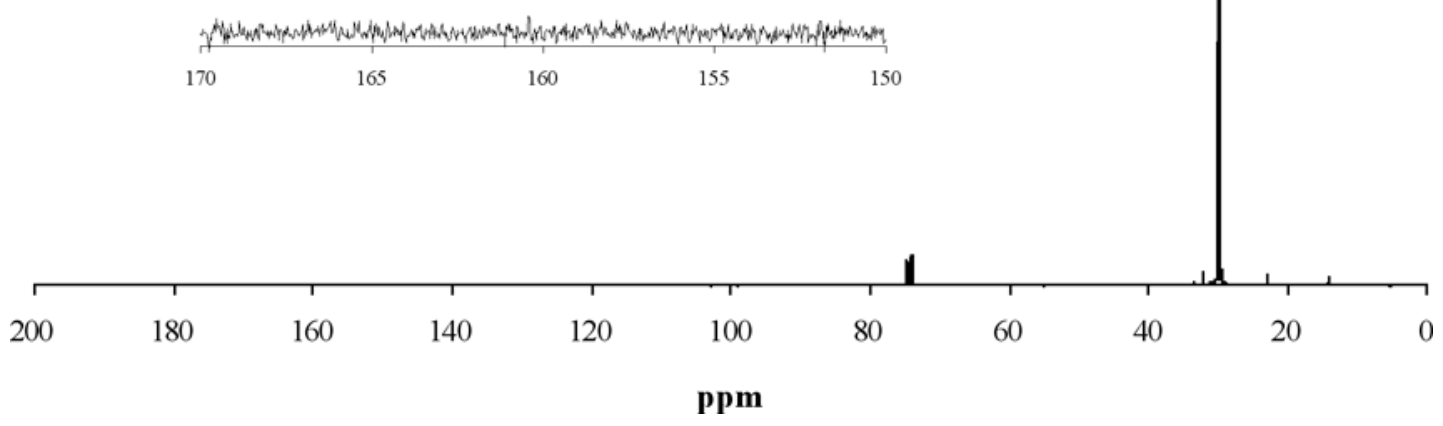


Polymer produced with 2/MAO (Table 5, entry 7), showing the absence of carbonyl peaks:

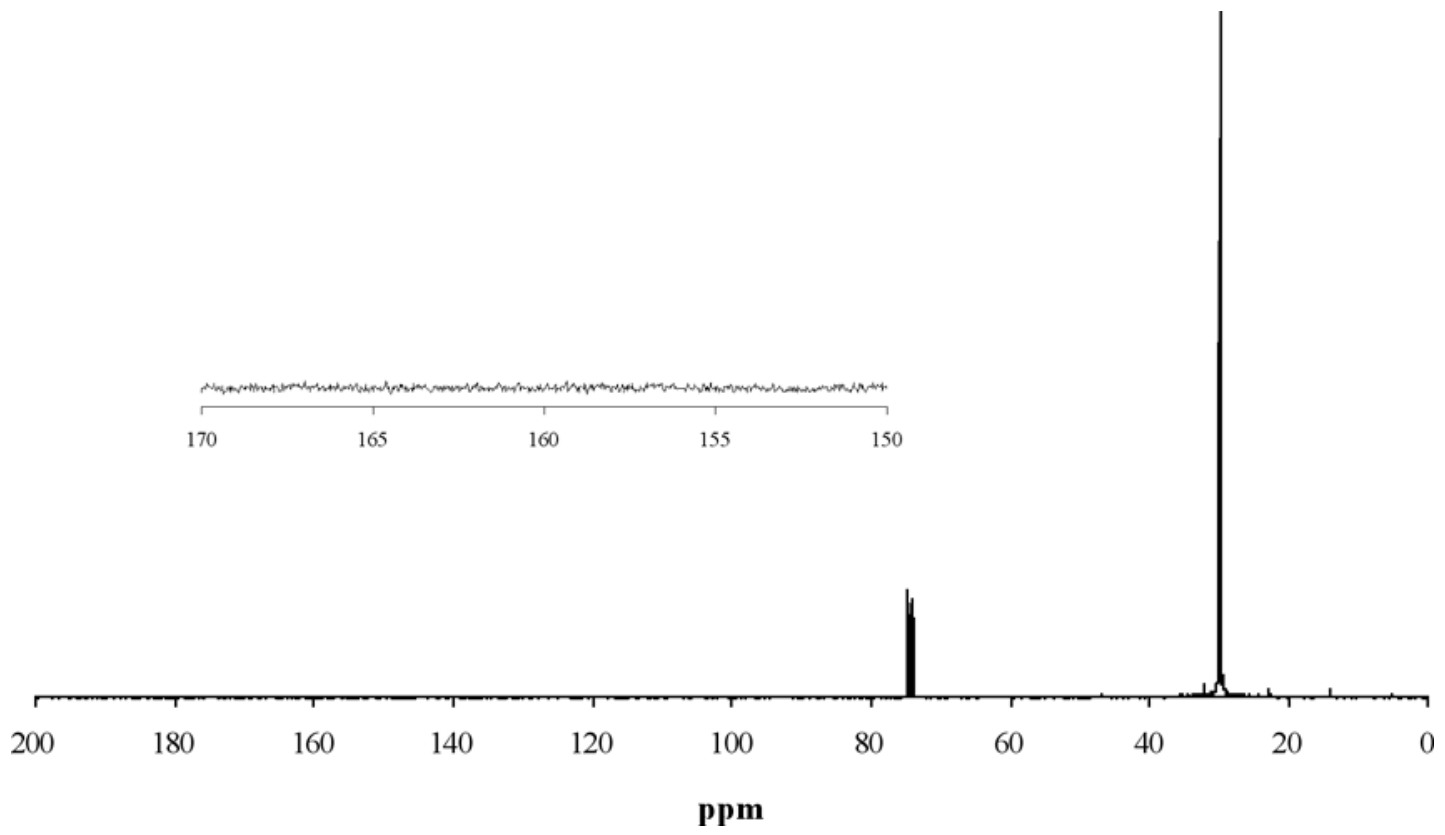

Polymer produced with 2/MAO (Table 5, entry 8), showing the absence of carbonyl peaks:
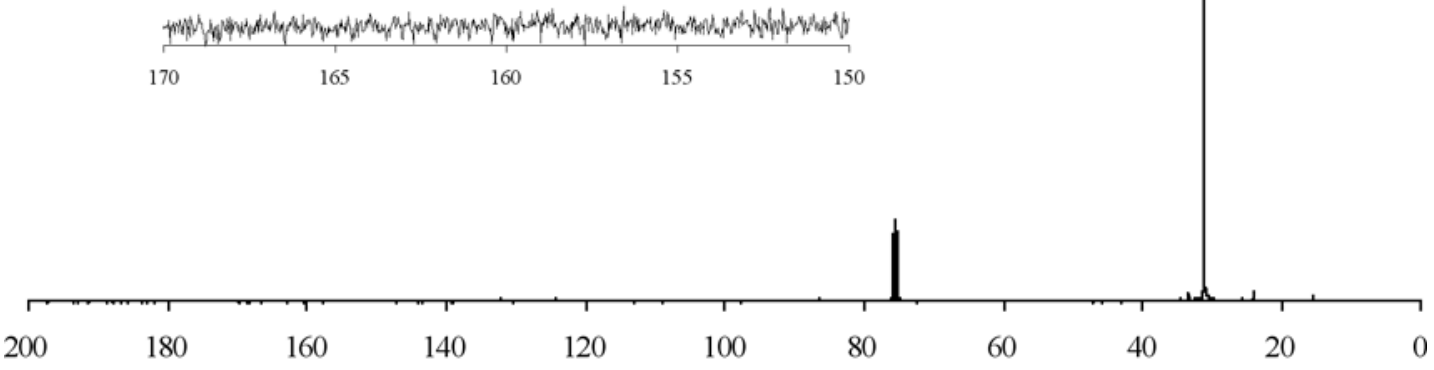

ppm 
Polymer produced by the homopolymerization of ethylene with $2 / \mathrm{MAO}$ (Table 5 , entry 9 ), showing the necessary absence of carbonyl peaks:

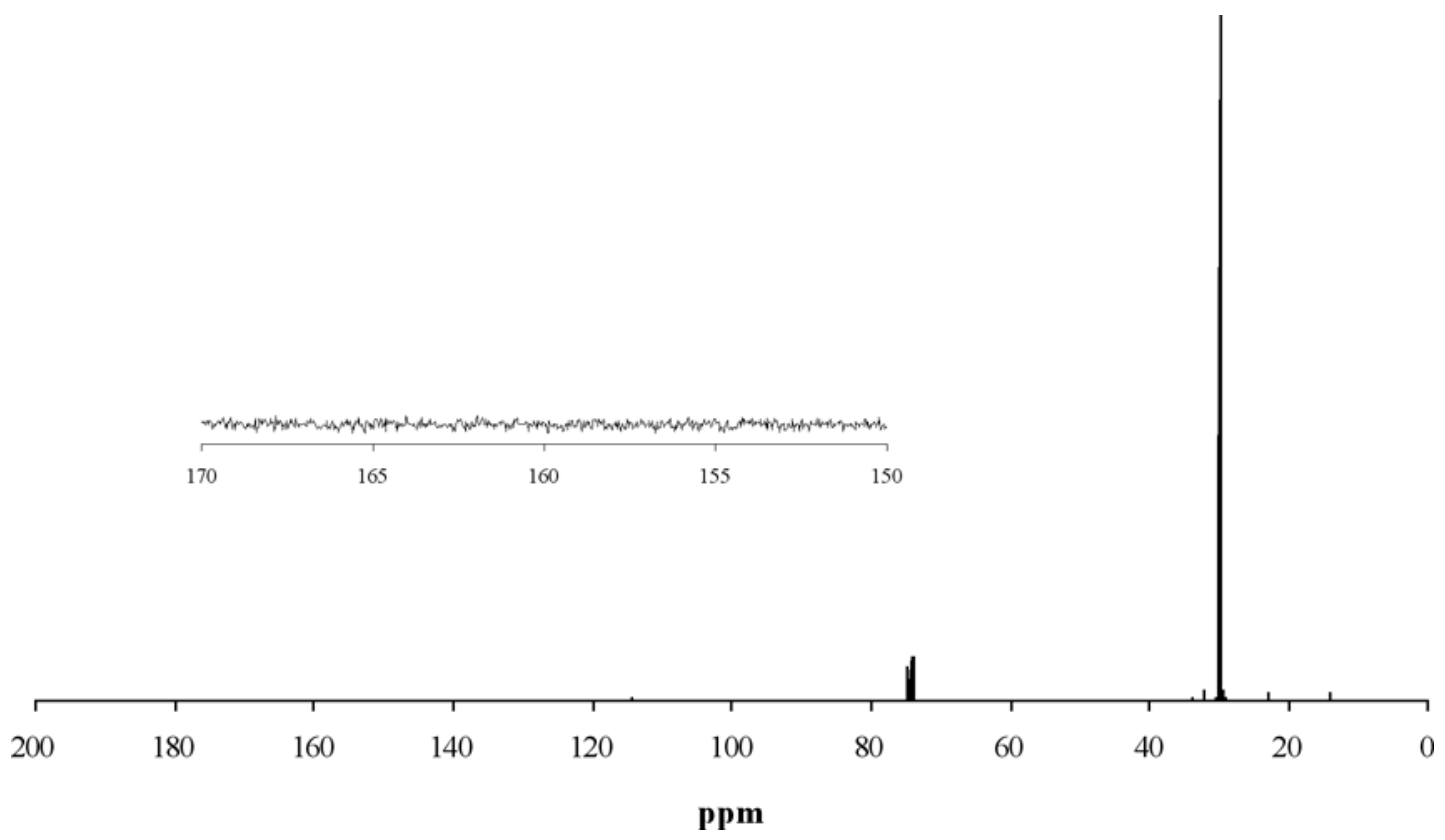

Polymer produced with $\mathbf{3} / \mathrm{MAO}$ (Table 5, entry 10), showing the absence of carbonyl peaks:

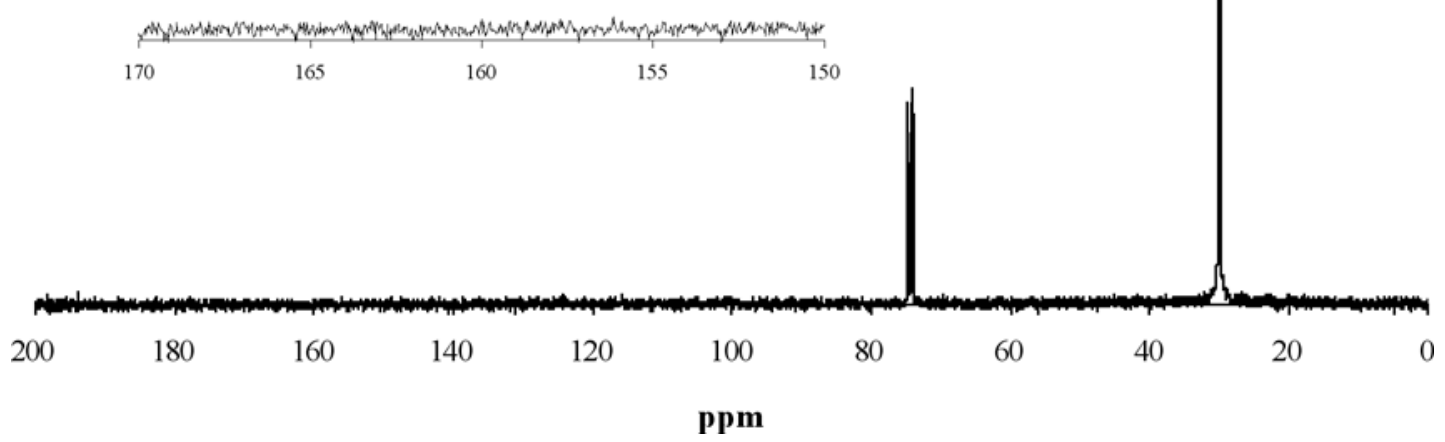


Polymer produced with $\mathbf{5} / \mathrm{MAO}$ (Table 5, entry 14), showing the absence of carbonyl peaks:

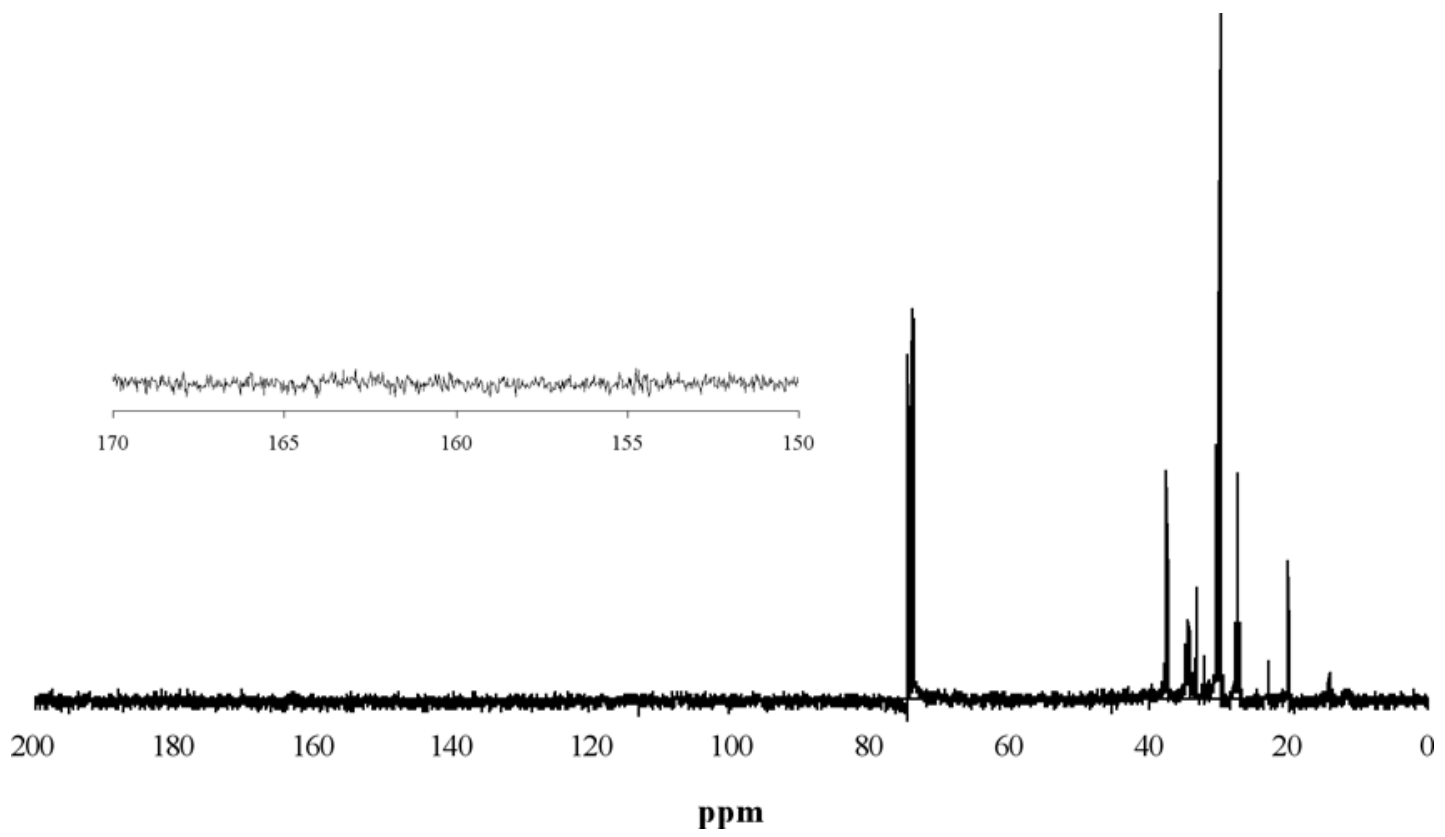

Polymer produced with 5/MAO, (Table 5, entry 15), showing the absence of carbonyl peaks:

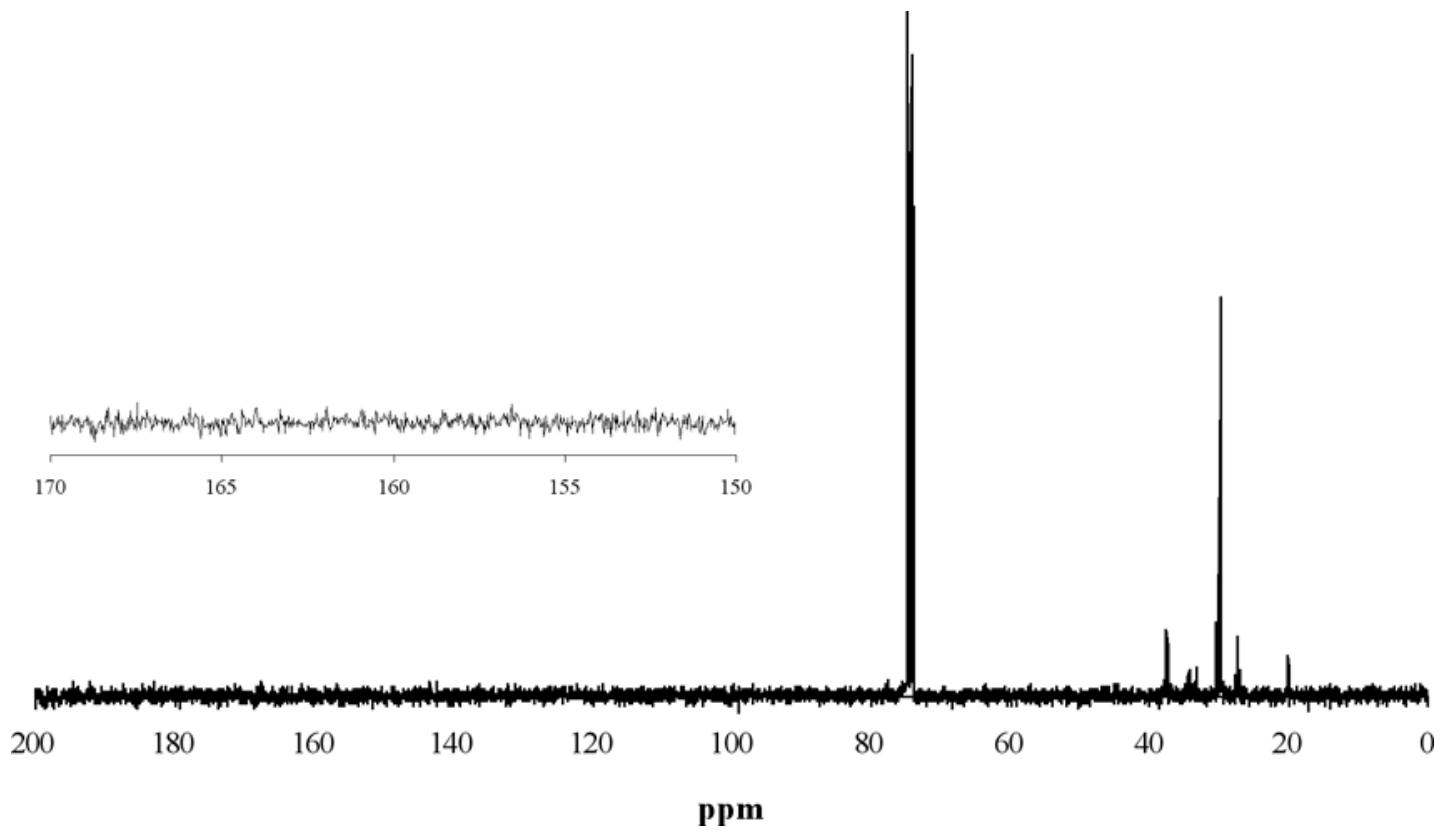




\section{GC-MS Spectra}

GC traces of oligomers produced with 4/MAO, (Table 5, entry 12), with the representative masses for each peak:

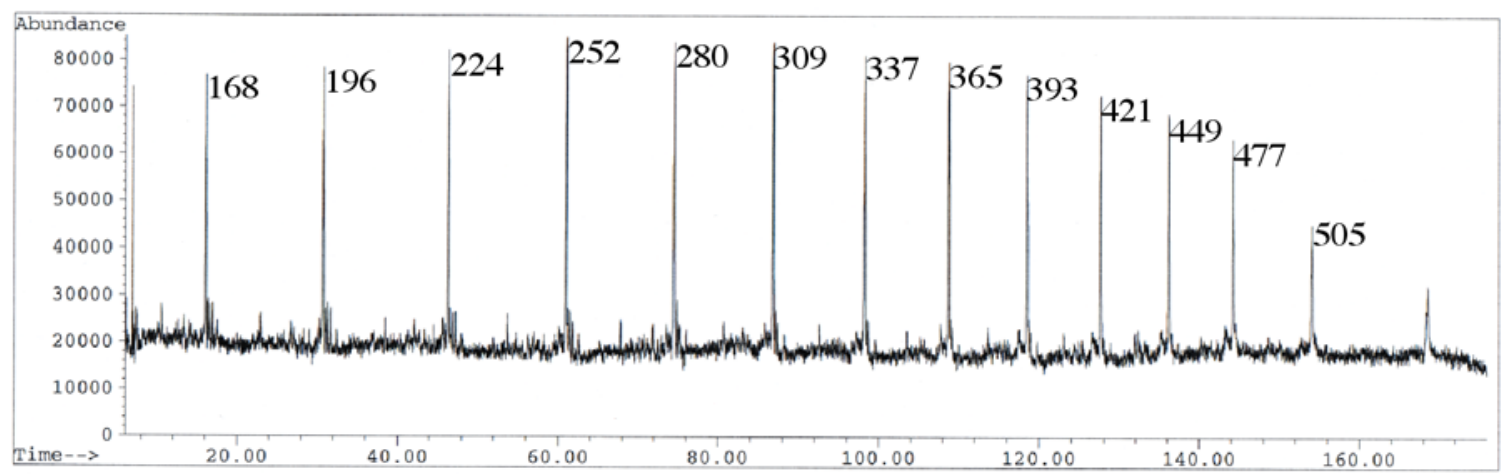

GC traces of oligomers produced with 4/MAO, (Table 5, entry 13), with the representative masses for each peak:

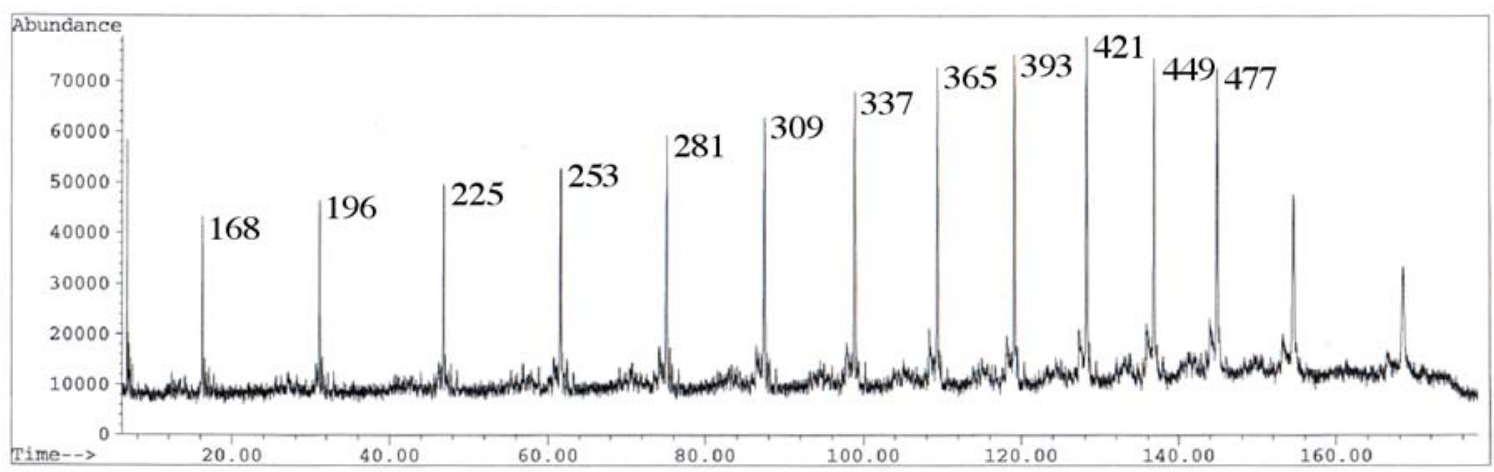

GC traces of oligomers produced with 6/MAO, (Table 5, entry 16), with the representative masses for each peak:

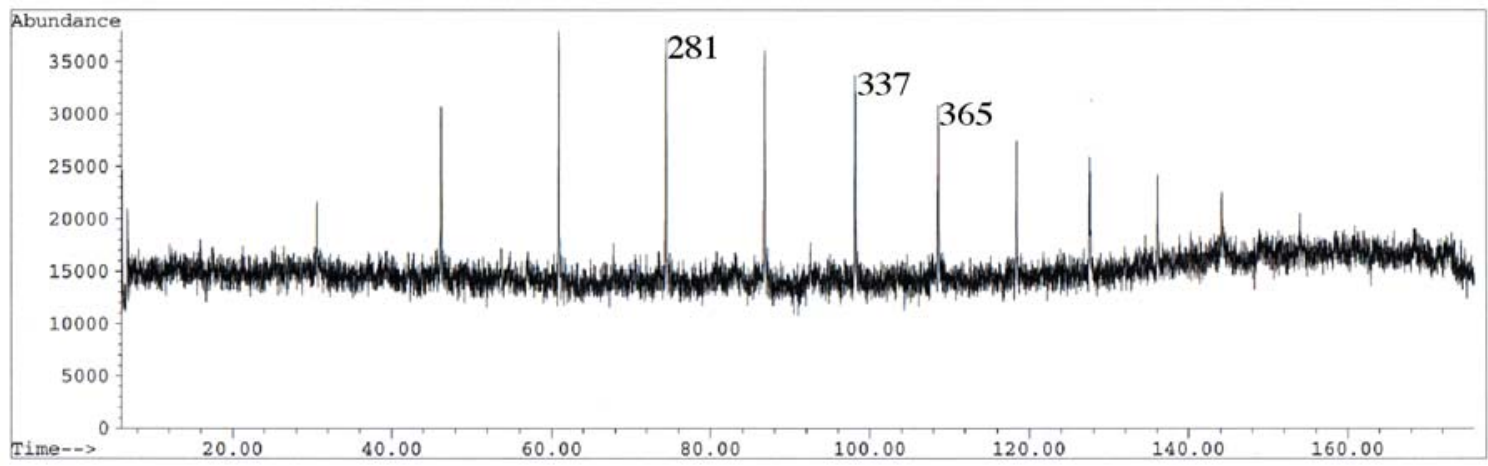




\section{Computational Methods}

All calculations were performed using the Gaussian 03 suite of programs. ${ }^{7}$ Optimized structure calculations and frequency calculations were performed using Density Function Theory (DFT) employing the Becke's 3parameter hybrid functional (B3) ${ }^{8}$ with the correlation functional of Lee, Yang and Parr (LYP). ${ }^{9,10}$ The Pople style basis set, $6-31 \mathrm{G}\left(\mathrm{d}^{\prime}\right)(=6-31 \mathrm{G} \dagger)$ was used. The compounds are named in the following manner: $X \operatorname{chain} Y$ where $X$ is the total number of monomer units in the chain and $Y$ is the number of $\mathrm{CO}_{2}$ units in the chain.

\section{DFT Structures and Coordinates}

\section{2chain0}

$\begin{array}{lrrr} & & & \\ & & & \\ \mathrm{C} & -0.7048 & -1.8364 & 0.0000 \\ \mathrm{C} & -0.7048 & -0.3036 & 0.0000 \\ \mathrm{C} & 0.7048 & 0.3036 & 0.0000 \\ \mathrm{C} & 0.7048 & 1.8364 & 0.0000 \\ \mathrm{H} & -1.7255 & -2.2399 & 0.0000 \\ \mathrm{H} & -0.1899 & -2.2337 & 0.8854 \\ \mathrm{H} & -0.1899 & -2.2337 & -0.8854 \\ \mathrm{H} & -1.2565 & 0.0640 & 0.8788 \\ \mathrm{H} & -1.2565 & 0.0640 & -0.8788 \\ \mathrm{H} & 1.2565 & -0.0640 & -0.8788 \\ \mathrm{H} & 1.2565 & -0.0640 & 0.8788 \\ \mathrm{H} & 1.7255 & 2.2399 & 0.0000 \\ \mathrm{H} & 0.1899 & 2.2337 & 0.8854 \\ \mathrm{H} & 0.1899 & 2.2337 & -0.8854\end{array}$

3chain 0

\begin{tabular}{|c|c|c|c|}
\hline C & -1.3953 & -2.9156 & 0.0000 \\
\hline $\mathrm{C}$ & -1.4059 & -1.3827 & 0.0000 \\
\hline $\mathrm{C}$ & 0.0000 & -0.7672 & 0.0000 \\
\hline $\mathrm{C}$ & 0.0000 & 0.7672 & 0.0000 \\
\hline $\mathrm{C}$ & 1.4059 & 1.3827 & 0.0000 \\
\hline $\mathrm{C}$ & 1.3953 & 2.9156 & 0.0000 \\
\hline $\mathrm{H}$ & -2.4136 & -3.3253 & 0.0000 \\
\hline $\mathrm{H}$ & -0.8779 & -3.3094 & 0.8854 \\
\hline $\mathrm{H}$ & -0.8779 & -3.3094 & -0.8854 \\
\hline $\mathrm{H}$ & -1.9608 & -1.0195 & 0.8785 \\
\hline $\mathrm{H}$ & -1.9608 & -1.0195 & -0.8785 \\
\hline $\mathrm{H}$ & 0.5556 & -1.1316 & -0.8789 \\
\hline $\mathrm{H}$ & 0.5556 & -1.1316 & 0.8789 \\
\hline $\mathrm{H}$ & -0.5556 & 1.1316 & 0.8789 \\
\hline $\mathrm{H}$ & -0.5556 & 1.1316 & -0.8789 \\
\hline $\mathrm{H}$ & 1.9608 & 1.0195 & -0.8785 \\
\hline $\mathrm{H}$ & 1.9608 & 1.0195 & 0.8785 \\
\hline $\mathrm{H}$ & 2.4136 & 3.3253 & 0.0000 \\
\hline $\mathrm{H}$ & 0.8779 & 3.3094 & 0.8854 \\
\hline 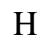 & 0.8779 & 3.3094 & -0.8854 \\
\hline
\end{tabular}




\begin{tabular}{|c|c|c|c|}
\hline \multicolumn{4}{|c|}{ 3chain 1} \\
\hline $\mathrm{C}$ & 3.0159 & -0.1625 & 0.0008 \\
\hline $\mathrm{C}$ & 1.6362 & -0.8196 & -0.0007 \\
\hline $\mathrm{C}$ & 0.5033 & 0.1912 & -0.0003 \\
\hline $\mathrm{O}$ & -0.6974 & -0.4315 & 0.0001 \\
\hline $\mathrm{C}$ & -1.8571 & 0.4317 & 0.0002 \\
\hline $\mathrm{C}$ & -3.0882 & -0.4567 & 0.0002 \\
\hline $\mathrm{O}$ & 0.6320 & 1.3935 & -0.0004 \\
\hline $\mathrm{H}$ & 3.8039 & -0.9248 & 0.0003 \\
\hline $\mathrm{H}$ & 3.1489 & 0.4719 & 0.8840 \\
\hline $\mathrm{H}$ & 3.1498 & 0.4738 & -0.8810 \\
\hline $\mathrm{H}$ & 1.5011 & -1.4735 & 0.8722 \\
\hline $\mathrm{H}$ & 1.5021 & -1.4715 & -0.8752 \\
\hline $\mathrm{H}$ & -1.8158 & 1.0798 & -0.8835 \\
\hline $\mathrm{H}$ & -1.8158 & 1.0797 & 0.8840 \\
\hline $\mathrm{H}$ & -3.9945 & 0.1613 & 0.0003 \\
\hline $\mathrm{H}$ & -3.1084 & -1.0987 & 0.8886 \\
\hline $\mathrm{H}$ & -3.1085 & -1.0985 & -0.8883 \\
\hline \multicolumn{4}{|c|}{ 4chain0 } \\
\hline $\mathrm{C}$ & 2.8131 & -3.5239 & 0.0000 \\
\hline $\mathrm{C}$ & 1.4056 & -2.9171 & 0.0000 \\
\hline $\mathrm{C}$ & 1.4056 & -1.3825 & 0.0000 \\
\hline $\mathrm{C}$ & -0.0001 & -0.7673 & 0.0000 \\
\hline $\mathrm{C}$ & 0.0001 & 0.7673 & 0.0000 \\
\hline $\mathrm{C}$ & -1.4056 & 1.3825 & 0.0000 \\
\hline $\mathrm{C}$ & -1.4056 & 2.9171 & 0.0000 \\
\hline $\mathrm{C}$ & -2.8131 & 3.5239 & 0.0000 \\
\hline $\mathrm{H}$ & 2.7780 & -4.6209 & 0.0000 \\
\hline $\mathrm{H}$ & 3.3822 & -3.2092 & 0.8854 \\
\hline $\mathrm{H}$ & 3.3822 & -3.2092 & -0.8854 \\
\hline $\mathrm{H}$ & 0.8498 & -3.2795 & 0.8784 \\
\hline $\mathrm{H}$ & 0.8498 & -3.2795 & -0.8784 \\
\hline $\mathrm{H}$ & 1.9625 & -1.0196 & -0.8787 \\
\hline $\mathrm{H}$ & 1.9625 & -1.0196 & 0.8787 \\
\hline $\mathrm{H}$ & -0.5563 & -1.1310 & 0.8787 \\
\hline $\mathrm{H}$ & -0.5563 & -1.1310 & -0.8787 \\
\hline $\mathrm{H}$ & 0.5563 & 1.1310 & -0.8787 \\
\hline $\mathrm{H}$ & 0.5563 & 1.1310 & 0.8787 \\
\hline $\mathrm{H}$ & -1.9625 & 1.0196 & 0.8787 \\
\hline $\mathrm{H}$ & -1.9625 & 1.0196 & -0.8787 \\
\hline $\mathrm{H}$ & -0.8498 & 3.2795 & -0.8784 \\
\hline $\mathrm{H}$ & -0.8498 & 3.2795 & 0.8784 \\
\hline $\mathrm{H}$ & -2.7780 & 4.6209 & 0.0000 \\
\hline $\mathrm{H}$ & -3.3822 & 3.2092 & 0.8854 \\
\hline $\mathrm{H}$ & -3.3822 & 3.2092 & -0.8854 \\
\hline
\end{tabular}


5chain0

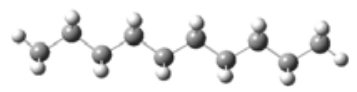

$\begin{array}{lrrr}\mathrm{C} & 2.8008 & 5.0651 & 0.0000 \\ \mathrm{C} & 2.8118 & 3.5321 & 0.0000 \\ \mathrm{C} & 1.4061 & 2.9164 & 0.0000 \\ \mathrm{C} & 1.4064 & 1.3817 & 0.0000 \\ \mathrm{C} & 0.0000 & 0.7673 & 0.0000 \\ \mathrm{C} & 0.0002 & -0.7675 & 0.0000 \\ \mathrm{C} & -1.4062 & -1.3818 & 0.0000 \\ \mathrm{C} & -1.4060 & -2.9165 & 0.0000 \\ \mathrm{C} & -2.8118 & -3.5320 & 0.0000 \\ \mathrm{C} & -2.8011 & -5.0649 & 0.0000 \\ \mathrm{H} & 3.8188 & 5.4753 & 0.0000 \\ \mathrm{H} & 2.2831 & 5.4587 & 0.8854 \\ \mathrm{H} & 2.2831 & 5.4587 & -0.8854 \\ \mathrm{H} & 3.3669 & 3.1692 & 0.8785 \\ \mathrm{H} & 3.3669 & 3.1692 & -0.8785 \\ \mathrm{H} & 0.8504 & 3.2807 & -0.8788 \\ \mathrm{H} & 0.8504 & 3.2807 & 0.8788 \\ \mathrm{H} & 1.9623 & 1.0181 & 0.8788 \\ \mathrm{H} & 1.9623 & 1.0181 & -0.8788 \\ \mathrm{H} & -0.5559 & 1.1310 & -0.8788 \\ \mathrm{H} & -0.5559 & 1.1310 & 0.8788 \\ \mathrm{H} & 0.5561 & -1.1312 & 0.8788 \\ \mathrm{H} & 0.5561 & -1.1312 & -0.8788 \\ \mathrm{H} & -1.9621 & -1.0182 & -0.8788 \\ \mathrm{H} & -1.9621 & -1.0182 & 0.8788 \\ \mathrm{H} & -0.8503 & -3.2808 & 0.8788 \\ \mathrm{H} & -0.8503 & -3.2808 & -0.8788 \\ \mathrm{H} & -3.3668 & -3.1689 & -0.8785 \\ \mathrm{H} & -3.3668 & -3.1689 & 0.8785 \\ \mathrm{H} & -3.8193 & -5.4748 & 0.0000 \\ \mathrm{H} & -2.2836 & -5.4587 & 0.8854 \\ \mathrm{H} & -2.2836 & -5.4587 & -0.8854\end{array}$

5chain 1

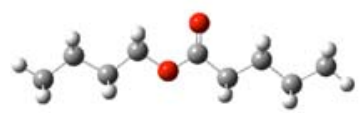

$\begin{array}{lrrr}\text { C } & -3.0480 & 0.1723 & 0.0001 \\ \text { C } & -1.6988 & -0.5500 & -0.0003 \\ \text { C } & -0.5135 & 0.3986 & -0.0001 \\ \text { O } & 0.6520 & -0.2881 & 0.0001 \\ \text { C } & 1.8565 & 0.5104 & 0.0001 \\ \text { C } & 3.0449 & -0.4396 & 0.0001 \\ \text { O } & -0.5761 & 1.6062 & -0.0002 \\ \text { C } & -4.2398 & -0.7930 & -0.0002 \\ \text { C } & -5.5926 & -0.0723 & 0.0004 \\ \text { C } & 4.3865 & 0.3062 & -0.0001 \\ \text { C } & 5.5927 & -0.6396 & 0.0001\end{array}$




$\begin{array}{rrrr}\mathrm{H} & -3.1026 & 0.8347 & -0.8748 \\ \mathrm{H} & -3.1025 & 0.8340 & 0.8754 \\ \mathrm{H} & -1.6004 & -1.2103 & -0.8743 \\ \mathrm{H} & -1.6003 & -1.2110 & 0.8732 \\ \mathrm{H} & 1.8517 & 1.1609 & 0.8840 \\ \mathrm{H} & 1.8518 & 1.1609 & -0.8837 \\ \mathrm{H} & 2.9794 & -1.0945 & -0.8805 \\ \mathrm{H} & 2.9796 & -1.0943 & 0.8810 \\ \mathrm{H} & -4.1764 & -1.4540 & 0.8780 \\ \mathrm{H} & -4.1767 & -1.4531 & -0.8792 \\ \mathrm{H} & -6.4264 & -0.7858 & -0.0001 \\ \mathrm{H} & -5.7015 & 0.5689 & -0.8845 \\ \mathrm{H} & -5.7013 & 0.5677 & 0.8862 \\ \mathrm{H} & 4.4383 & 0.9666 & 0.8786 \\ \mathrm{H} & 4.4382 & 0.9662 & -0.8791 \\ \mathrm{H} & 6.5371 & -0.0812 & -0.0002 \\ \mathrm{H} & 5.5885 & -1.2891 & -0.8854 \\ \mathrm{H} & 5.5887 & -1.2886 & 0.8859\end{array}$

6chain0

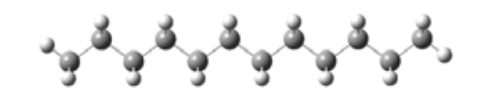

$\begin{array}{rrrr}\mathrm{C} & -4.2201 & 5.6727 & 0.0000 \\ \mathrm{C} & -2.8123 & 5.0664 & 0.0000 \\ \mathrm{C} & -2.8117 & 3.5318 & 0.0000 \\ \mathrm{C} & -1.4059 & 2.9170 & 0.0000 \\ \mathrm{C} & -1.4059 & 1.3823 & 0.0000 \\ \mathrm{C} & 0.0002 & 0.7674 & 0.0000 \\ \mathrm{C} & -0.0002 & -0.7674 & 0.0000 \\ \mathrm{C} & 1.4059 & -1.3823 & 0.0000 \\ \mathrm{C} & 1.4059 & -2.9170 & 0.0000 \\ \mathrm{C} & 2.8117 & -3.5318 & 0.0000 \\ \mathrm{C} & 2.8123 & -5.0664 & 0.0000 \\ \mathrm{C} & 4.2201 & -5.6727 & 0.0000 \\ \mathrm{H} & -4.1853 & 6.7698 & 0.0000 \\ \mathrm{H} & -4.7891 & 5.3580 & 0.8854 \\ \mathrm{H} & -4.7891 & 5.3580 & -0.8854 \\ \mathrm{H} & -2.2570 & 5.4292 & 0.8785 \\ \mathrm{H} & -2.2570 & 5.4292 & -0.8785 \\ \mathrm{H} & -3.3683 & 3.1688 & -0.8788 \\ \mathrm{H} & -3.3683 & 3.1688 & 0.8788 \\ \mathrm{H} & -0.8500 & 3.2808 & 0.8788 \\ \mathrm{H} & -0.8500 & 3.2808 & -0.8788 \\ \mathrm{H} & -1.9618 & 1.0186 & -0.8788 \\ \mathrm{H} & -1.9618 & 1.0186 & 0.8788 \\ \mathrm{H} & 0.5561 & 1.1310 & 0.8788 \\ \mathrm{H} & 0.5561 & 1.1310 & -0.8788 \\ \mathrm{H} & -0.5561 & -1.1310 & -0.8788 \\ \mathrm{H} & -0.5561 & -1.1310 & 0.8788 \\ \mathrm{H} & 1.9618 & -1.0186 & 0.8788 \\ \mathrm{H} & 1.9618 & -1.0186 & -0.8788 \\ \mathrm{H} & 0.8500 & -3.2808 & -0.8788 \\ \mathrm{H} & 0.8500 & -3.2808 & 0.8788\end{array}$




$\begin{array}{lrrr}\mathrm{H} & 3.3683 & -3.1688 & 0.8788 \\ \mathrm{H} & 3.3683 & -3.1688 & -0.8788 \\ \mathrm{H} & 2.2570 & -5.4292 & -0.8785 \\ \mathrm{H} & 2.2570 & -5.4292 & 0.8785 \\ \mathrm{H} & 4.1853 & -6.7698 & 0.0000 \\ \mathrm{H} & 4.7891 & -5.3580 & 0.8854 \\ \mathrm{H} & 4.7891 & -5.3580 & -0.8854\end{array}$

7chain0

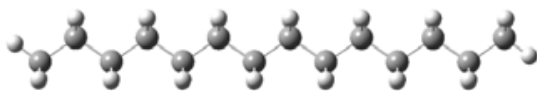

$\begin{array}{llll}\text { C } & -8.3458 & -0.3237 & -0.0005\end{array}$

$\begin{array}{llll}\text { C } & -7.0580 & 0.5077 & -0.0005\end{array}$

$\begin{array}{llll}\text { C } & -5.7838 & -0.3476 & -0.0003\end{array}$

$\begin{array}{llll}\text { C } & -4.4893 & 0.4765 & -0.0003\end{array}$

$\begin{array}{llll}\text { C } & -3.2155 & -0.3796 & -0.0001\end{array}$

$\begin{array}{llll}\text { C } & -1.9209 & 0.4446 & -0.0001\end{array}$

$\begin{array}{llll}\text { C } & -0.6474 & -0.4117 & 0.0000\end{array}$

$\begin{array}{llll}\text { C } & 0.6474 & 0.4123 & 0.0000\end{array}$

$\begin{array}{llll}\text { C } & 1.9208 & -0.4442 & 0.0002\end{array}$

$\begin{array}{llll}\text { C } & 3.2156 & 0.3799 & 0.0001\end{array}$

$\begin{array}{llll}\text { C } & 4.4892 & -0.4764 & 0.0003\end{array}$

$\begin{array}{llll}\text { C } & 5.7839 & 0.3474 & 0.0003\end{array}$

$\begin{array}{llll}\text { C } & 7.0579 & -0.5082 & 0.0004\end{array}$

$\begin{array}{llll}\text { C } & 8.3459 & 0.3230 & 0.0004\end{array}$

$\begin{array}{llll}\mathrm{H} & -9.2378 & 0.3159 & -0.0006\end{array}$

$\begin{array}{llll}\mathrm{H} & -8.4008 & -0.9715 & 0.8850\end{array}$

$\begin{array}{llll}\mathrm{H} & -8.4007 & -0.9717 & -0.8858\end{array}$

$\begin{array}{llll}\mathrm{H} & -7.0494 & 1.1710 & 0.8780\end{array}$

$\begin{array}{llll}\mathrm{H} & -7.0493 & 1.1709 & -0.8790\end{array}$

$\begin{array}{llll}\mathrm{H} & -5.7928 & -1.0121 & -0.8790\end{array}$

$\begin{array}{llll}\mathrm{H} & -5.7929 & -1.0120 & 0.8785\end{array}$

$\begin{array}{llll}\mathrm{H} & -4.4812 & 1.1409 & 0.8784\end{array}$

$\begin{array}{llll}\mathrm{H} & -4.4811 & 1.1407 & -0.8791\end{array}$

$\begin{array}{llll}\mathrm{H} & -3.2237 & -1.0439 & -0.8788\end{array}$

$\begin{array}{llll}\mathrm{H} & -3.2238 & -1.0438 & 0.8787\end{array}$

$\begin{array}{llll}\mathrm{H} & -1.9127 & 1.1090 & 0.8786\end{array}$

$\begin{array}{llll}\mathrm{H} & -1.9126 & 1.1088 & -0.8789\end{array}$

$\begin{array}{llll}\mathrm{H} & -0.6556 & -1.0761 & -0.8786\end{array}$

$\begin{array}{llll}\mathrm{H} & -0.6557 & -1.0760 & 0.8788\end{array}$

$\begin{array}{llll}\mathrm{H} & 0.6557 & 1.0766 & 0.8787\end{array}$

$\begin{array}{llll}\mathrm{H} & 0.6558 & 1.0765 & -0.8788\end{array}$

$\begin{array}{llll}\mathrm{H} & 1.9125 & -1.1085 & -0.8785\end{array}$

$\begin{array}{llll}\mathrm{H} & 1.9125 & -1.1083 & 0.8790\end{array}$

$\begin{array}{llll}\mathrm{H} & 3.2239 & 1.0443 & 0.8788\end{array}$

$\begin{array}{llll}\mathrm{H} & 3.2240 & 1.0441 & -0.8786\end{array}$

$\begin{array}{llll}\mathrm{H} & 4.4810 & -1.1408 & -0.8784\end{array}$

$\begin{array}{llll}\mathrm{H} & 4.4809 & -1.1406 & 0.8791\end{array}$

$\begin{array}{llll}\mathrm{H} & 5.7931 & 1.0120 & 0.8790\end{array}$

$\begin{array}{llll}\mathrm{H} & 5.7931 & 1.0118 & -0.8786\end{array}$

$\begin{array}{llll}\mathrm{H} & 7.0492 & -1.1715 & -0.8780\end{array}$

$\begin{array}{llll}\mathrm{H} & 7.0491 & -1.1713 & 0.8789\end{array}$

$\begin{array}{llll}\mathrm{H} & 9.2377 & -0.3168 & 0.0005\end{array}$ 


$\begin{array}{lllr}\mathrm{H} & 8.4010 & 0.9710 & 0.8857 \\ \mathrm{H} & 8.4010 & 0.9708 & -0.8851\end{array}$

7chain 1

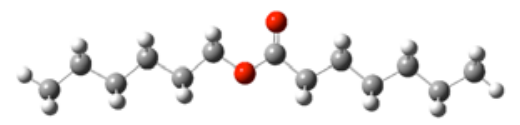

$\begin{array}{lrrr} & & & \\ \mathrm{C} & 3.0550 & 0.3428 & 0.0000 \\ \mathrm{C} & 1.7134 & -0.3939 & -0.0002 \\ \mathrm{C} & 0.5186 & 0.5427 & -0.0001 \\ \mathrm{O} & -0.6402 & -0.1554 & -0.0001 \\ \mathrm{C} & -1.8523 & 0.6316 & 0.0000 \\ \mathrm{C} & -3.0321 & -0.3292 & -0.0001 \\ \mathrm{O} & 0.5692 & 1.7509 & 0.0000 \\ \mathrm{C} & 4.2571 & -0.6099 & -0.0002 \\ \mathrm{C} & 5.6073 & 0.1191 & 0.0001 \\ \mathrm{C} & -4.3795 & 0.4061 & 0.0000 \\ \mathrm{C} & -5.5857 & -0.5425 & 0.0001 \\ \mathrm{C} & 6.8158 & -0.8264 & 0.0000 \\ \mathrm{C} & 8.1604 & -0.0903 & 0.0003 \\ \mathrm{C} & -6.9362 & 0.1859 & 0.0000 \\ \mathrm{C} & -8.1368 & -0.7670 & 0.0002 \\ \mathrm{H} & 3.1015 & 1.0052 & 0.8752 \\ \mathrm{H} & 3.1016 & 1.0055 & -0.8749 \\ \mathrm{H} & 1.6218 & -1.0557 & 0.8734 \\ \mathrm{H} & 1.6219 & -1.0554 & -0.8740 \\ \mathrm{H} & -1.8536 & 1.2823 & -0.8838 \\ \mathrm{H} & -1.8535 & 1.2822 & 0.8838 \\ \mathrm{H} & -2.9600 & -0.9832 & 0.8806 \\ \mathrm{H} & -2.9601 & -0.9831 & -0.8808 \\ \mathrm{H} & 4.2016 & -1.2719 & -0.8792 \\ \mathrm{H} & 4.2015 & -1.2724 & 0.8785 \\ \mathrm{H} & 5.6632 & 0.7808 & 0.8790 \\ \mathrm{H} & 5.6633 & 0.7813 & -0.8783 \\ \mathrm{H} & -4.4370 & 1.0670 & -0.8791 \\ \mathrm{H} & -4.4369 & 1.0670 & 0.8790 \\ \mathrm{H} & -5.5290 & -1.2038 & 0.8791 \\ \mathrm{H} & -5.5290 & -1.2040 & -0.8788 \\ \mathrm{H} & 6.7597 & -1.4870 & -0.8788 \\ \mathrm{H} & 6.7596 & -1.4875 & 0.8783 \\ \mathrm{H} & 9.0031 & -0.7934 & 0.0001 \\ \mathrm{H} & 8.2624 & 0.5515 & 0.8859 \\ \mathrm{H} & 8.2625 & 0.5520 & -0.8849 \\ \mathrm{H} & -6.9919 & 0.8466 & -0.8786 \\ \mathrm{H} & -6.9918 & 0.8468 & 0.8785 \\ & -9.0860 & -0.2164 & 0.0001 \\ & -8.1289 & -1.4168 & 0.8858 \\ & -1.4171 & -0.8852\end{array}$


schain0

\begin{tabular}{|c|c|c|c|}
\hline $\mathrm{C}$ & 5.6318 & -7.8173 & 0.0000 \\
\hline $\mathrm{C}$ & 4.2227 & -7.2136 & 0.0000 \\
\hline $\mathrm{C}$ & 4.2209 & -5.6788 & 0.0000 \\
\hline $\mathrm{C}$ & 2.8142 & -5.0653 & 0.0000 \\
\hline $\mathrm{C}$ & 2.8136 & -3.5304 & 0.0000 \\
\hline $\mathrm{C}$ & 1.4068 & -2.9165 & 0.0000 \\
\hline $\mathrm{C}$ & 1.4067 & -1.3816 & 0.0000 \\
\hline $\mathrm{C}$ & 0.0000 & -0.7675 & 0.0000 \\
\hline $\mathrm{C}$ & 0.0000 & 0.7675 & 0.0000 \\
\hline $\mathrm{C}$ & -1.4067 & 1.3816 & 0.0000 \\
\hline $\mathrm{C}$ & -1.4068 & 2.9165 & 0.0000 \\
\hline $\mathrm{C}$ & -2.8136 & 3.5304 & 0.0000 \\
\hline $\mathrm{C}$ & -2.8142 & 5.0653 & 0.0000 \\
\hline $\mathrm{C}$ & -4.2209 & 5.6788 & 0.0000 \\
\hline $\mathrm{C}$ & -4.2227 & 7.2136 & 0.0000 \\
\hline $\mathrm{C}$ & -5.6318 & 7.8173 & 0.0000 \\
\hline $\mathrm{H}$ & 5.5995 & -8.9145 & 0.0000 \\
\hline $\mathrm{H}$ & 6.1998 & -7.5009 & 0.8854 \\
\hline $\mathrm{H}$ & 6.1998 & -7.5009 & -0.8854 \\
\hline $\mathrm{H}$ & 3.6678 & -7.5768 & 0.8785 \\
\hline $\mathrm{H}$ & 3.6678 & -7.5768 & -0.8785 \\
\hline $\mathrm{H}$ & 4.7771 & -5.3154 & -0.8788 \\
\hline $\mathrm{H}$ & 4.7771 & -5.3154 & 0.8788 \\
\hline $\mathrm{H}$ & 2.2586 & -5.4293 & 0.8788 \\
\hline $\mathrm{H}$ & 2.2586 & -5.4293 & -0.8788 \\
\hline $\mathrm{H}$ & 3.3692 & -3.1665 & -0.8788 \\
\hline $\mathrm{H}$ & 3.3692 & -3.1665 & 0.8788 \\
\hline $\mathrm{H}$ & 0.8511 & -3.2803 & 0.8788 \\
\hline $\mathrm{H}$ & 0.8511 & -3.2803 & -0.8788 \\
\hline $\mathrm{H}$ & 1.9624 & -1.0178 & -0.8788 \\
\hline $\mathrm{H}$ & 1.9624 & -1.0178 & 0.8788 \\
\hline $\mathrm{H}$ & -0.5557 & -1.1312 & 0.8788 \\
\hline $\mathrm{H}$ & -0.5557 & -1.1312 & -0.8788 \\
\hline $\mathrm{H}$ & 0.5557 & 1.1312 & -0.8788 \\
\hline $\mathrm{H}$ & 0.5557 & 1.1312 & 0.8788 \\
\hline $\mathrm{H}$ & -1.9624 & 1.0178 & 0.8788 \\
\hline $\mathrm{H}$ & -1.9624 & 1.0178 & -0.8788 \\
\hline $\mathrm{H}$ & -0.8511 & 3.2803 & -0.8788 \\
\hline $\mathrm{H}$ & -0.8511 & 3.2803 & 0.8788 \\
\hline $\mathrm{H}$ & -3.3692 & 3.1665 & 0.8788 \\
\hline $\mathrm{H}$ & -3.3692 & 3.1665 & -0.8788 \\
\hline $\mathrm{H}$ & -2.2586 & 5.4293 & -0.8788 \\
\hline $\mathrm{H}$ & -2.2586 & 5.4293 & 0.8788 \\
\hline $\mathrm{H}$ & -4.7771 & 5.3154 & 0.8788 \\
\hline $\mathrm{H}$ & -4.7771 & 5.3154 & -0.8788 \\
\hline $\mathrm{H}$ & -3.6678 & 7.5768 & -0.8785 \\
\hline $\mathrm{H}$ & -3.6678 & 7.5768 & 0.8785 \\
\hline $\mathrm{H}$ & -5.5995 & 8.9145 & 0.0000 \\
\hline $\mathrm{H}$ & -6.1998 & 7.5009 & 0.8854 \\
\hline $\mathrm{H}$ & -6.1998 & 7.5009 & -0.8854 \\
\hline
\end{tabular}


chain0

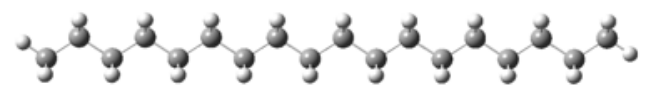

$\begin{array}{llll}\text { C } & 10.9125 & -0.3458 & -0.0001\end{array}$

$\begin{array}{llll}\text { C } & 9.6289 & 0.4922 & 0.0002\end{array}$

$\begin{array}{llll}\text { C } & 8.3504 & -0.3565 & 0.0003\end{array}$

$\begin{array}{llll}\text { C } & 7.0601 & 0.4741 & 0.0006\end{array}$

$\begin{array}{llll}\text { C } & 5.7821 & -0.3755 & 0.0006\end{array}$

$\begin{array}{llll}\text { C } & 4.4916 & 0.4552 & 0.0008\end{array}$

$\begin{array}{llll}\text { C } & 3.2138 & -0.3948 & 0.0008\end{array}$

$\begin{array}{llll}\text { C } & 1.9231 & 0.4355 & 0.0009\end{array}$

$\begin{array}{llll}\text { C } & 0.6454 & -0.4147 & 0.0007\end{array}$

$\begin{array}{llll}\text { C } & -0.6454 & 0.4155 & 0.0007\end{array}$

$\begin{array}{llll}\text { C } & -1.9230 & -0.4349 & 0.0004\end{array}$

$\begin{array}{llll}\text { C } & -3.2138 & 0.3954 & 0.0003\end{array}$

$\begin{array}{llll}\text { C } & -4.4915 & -0.4548 & -0.0002\end{array}$

$\begin{array}{llll}\text { C } & -5.7821 & 0.3756 & -0.0003\end{array}$

$\begin{array}{llll}\text { C } & -7.0600 & -0.4743 & -0.0008\end{array}$

$\begin{array}{llll}\text { C } & -8.3505 & 0.3561 & -0.0011\end{array}$

$\begin{array}{llll}\text { C } & -9.6288 & -0.4929 & -0.0016\end{array}$

$\begin{array}{llll}\text { C } & -10.9126 & 0.3448 & -0.0018\end{array}$

$\begin{array}{llll}\mathrm{H} & 11.8077 & 0.2892 & -0.0001\end{array}$

$\begin{array}{llll}\mathrm{H} & 10.9639 & -0.9939 & -0.8856\end{array}$

$\begin{array}{llll}\mathrm{H} & 10.9642 & -0.9941 & 0.8853\end{array}$

$\begin{array}{llll}\mathrm{H} & 9.6236 & 1.1556 & -0.8782\end{array}$

$\begin{array}{llll}\mathrm{H} & 9.6239 & 1.1554 & 0.8788\end{array}$

$\begin{array}{llll}\mathrm{H} & 8.3562 & -1.0211 & 0.8790\end{array}$

$\begin{array}{llll}\mathrm{H} & 8.3560 & -1.0209 & -0.8785\end{array}$

$\mathrm{H} \quad 7.0552 \quad 1.1386 \quad-0.8781$

$\begin{array}{llll}\mathrm{H} & 7.0554 & 1.1384 & 0.8794\end{array}$

$\begin{array}{llll}\mathrm{H} & 5.7870 & -1.0399 & 0.8793\end{array}$

$\begin{array}{llll}\mathrm{H} & 5.7869 & -1.0397 & -0.8782\end{array}$

$\begin{array}{llll}\mathrm{H} & 4.4865 & 1.1196 & -0.8779\end{array}$

$\begin{array}{llll}\mathrm{H} & 4.4866 & 1.1194 & 0.8796\end{array}$

$\begin{array}{llll}\mathrm{H} & 3.2189 & -1.0592 & 0.8794\end{array}$

$\begin{array}{llll}\mathrm{H} & 3.2188 & -1.0591 & -0.8780\end{array}$

$\begin{array}{llll}\mathrm{H} & 1.9180 & 1.1000 & -0.8778\end{array}$

$\begin{array}{llll}\mathrm{H} & 1.9179 & 1.0997 & 0.8797\end{array}$

$\begin{array}{llll}\mathrm{H} & 0.6505 & -1.0792 & 0.8793\end{array}$

$\begin{array}{llll}\mathrm{H} & 0.6506 & -1.0790 & -0.8781\end{array}$

$\begin{array}{llll}\mathrm{H} & -0.6505 & 1.0799 & -0.8780\end{array}$

$\begin{array}{llll}\mathrm{H} & -0.6507 & 1.0796 & 0.8795\end{array}$

$\begin{array}{llll}\mathrm{H} & -1.9180 & -1.0993 & 0.8790\end{array}$

$\begin{array}{llll}\mathrm{H} & -1.9177 & -1.0991 & -0.8784\end{array}$

$\begin{array}{rrrr}\mathrm{H} & -1.9177 & -1.0991 & -0.8784 \\ \mathrm{H} & -3.2188 & 1.0598 & -0.8784\end{array}$

$\begin{array}{llll}\mathrm{H} & -3.2191 & 1.0595 & 0.8791\end{array}$

$\begin{array}{llll}\mathrm{H} & -4.4866 & -1.1193 & 0.8785\end{array}$

$\mathrm{H} \quad-4.4862 \quad-1.1190 \quad-0.8790$

$\begin{array}{llll}\mathrm{H} & -5.7869 & 1.0401 & -0.8789\end{array}$

$\begin{array}{llll}\mathrm{H} & -5.7874 & 1.0398 & 0.8785\end{array}$

$\begin{array}{llll}\mathrm{H} & -7.0553 & -1.1387 & 0.8778\end{array}$

$\begin{array}{llll}\mathrm{H} & -7.0548 & -1.1384 & -0.8797\end{array}$

$\begin{array}{llll}\mathrm{H} & -8.3561 & 1.0208 & -0.8797\end{array}$ 


$\begin{array}{rrrr}\mathrm{H} & -8.3566 & 1.0205 & 0.8778 \\ \mathrm{H} & -9.6237 & -1.1564 & 0.8768 \\ \mathrm{H} & -9.6232 & -1.1560 & -0.8802 \\ \mathrm{H} & -11.8076 & -0.2905 & -0.0022 \\ \mathrm{H} & -10.9641 & 0.9931 & -0.8871 \\ \mathrm{H} & -10.9646 & 0.9928 & 0.8837\end{array}$

9chain1

$\begin{array}{rrrr} & & \\ \end{array}$




$\begin{array}{rrrr}\mathrm{H} & 9.3320 & -1.5435 & -0.8784 \\ \mathrm{H} & 9.3320 & -1.5434 & 0.8786 \\ \mathrm{H} & 11.5721 & -0.8379 & 0.0002 \\ \mathrm{H} & 10.8244 & 0.5033 & 0.8854 \\ \mathrm{H} & 10.8245 & 0.5032 & -0.8854 \\ \mathrm{H} & -9.5451 & 0.7387 & -0.8780 \\ \mathrm{H} & -9.5450 & 0.7379 & 0.8790 \\ \mathrm{H} & -11.6403 & -0.3236 & 0.0002 \\ \mathrm{H} & -10.6835 & -1.5250 & 0.8850 \\ \mathrm{H} & -10.6836 & -1.5242 & -0.8859\end{array}$

10chain0

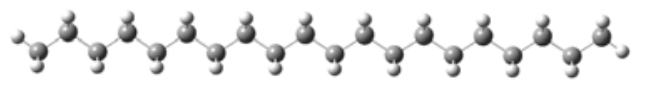

$\begin{array}{llll}\text { C } & 5.6279 & -7.8280 & 0.0000\end{array}$

$\begin{array}{llll}\text { C } & 4.2214 & -7.2146 & 0.0000\end{array}$

$\begin{array}{llll}\text { C } & 4.2198 & -5.6799 & 0.0000\end{array}$

$\begin{array}{llll}\text { C } & 2.8133 & -5.0661 & 0.0000\end{array}$

$\begin{array}{llll}\text { C } & 2.8125 & -3.5314 & 0.0000\end{array}$

$\begin{array}{llll}\text { C } & 1.4063 & -2.9169 & 0.0000\end{array}$

$\begin{array}{llll}\text { C } & 1.4061 & -1.3822 & 0.0000\end{array}$

$\begin{array}{llll}\text { C } & 0.0000 & -0.7673 & 0.0000\end{array}$

$\begin{array}{llll}\text { C } & 0.0000 & 0.7673 & 0.0000\end{array}$

$\begin{array}{llll}\text { C } & -1.4061 & 1.3822 & 0.0000\end{array}$

$\begin{array}{llll}\text { C } & -1.4063 & 2.9169 & 0.0000\end{array}$

$\begin{array}{llll}\text { C } & -2.8125 & 3.5314 & 0.0000\end{array}$

$\begin{array}{llll}\text { C } & -2.8133 & 5.0661 & 0.0000\end{array}$

$\begin{array}{llll}\text { C } & -4.2198 & 5.6799 & 0.0000\end{array}$

$\begin{array}{llll}\text { C } & -4.2214 & 7.2146 & 0.0000\end{array}$

$\begin{array}{llll}\text { C } & -5.6279 & 7.8280 & 0.0000\end{array}$

$\begin{array}{llll}\text { C } & 5.6306 & -9.3626 & 0.0000\end{array}$

$\begin{array}{llll}\text { C } & 7.0395 & -9.9663 & 0.0000\end{array}$

$\begin{array}{llll}\text { C } & -5.6305 & 9.3626 & 0.0000\end{array}$

$\begin{array}{llll}\text { C } & -7.0395 & 9.9663 & 0.0000\end{array}$

$\begin{array}{llll}\mathrm{H} & 6.1844 & -7.4642 & 0.8786\end{array}$

$\begin{array}{llll}\mathrm{H} & 6.1844 & -7.4642 & -0.8786\end{array}$

$\begin{array}{llll}\mathrm{H} & 3.6656 & -7.5790 & 0.8786\end{array}$

$\begin{array}{llll}\mathrm{H} & 3.6656 & -7.5790 & -0.8786\end{array}$

$\begin{array}{llll}\mathrm{H} & 4.7757 & -5.3156 & -0.8785\end{array}$

$\begin{array}{llll}\mathrm{H} & 4.7757 & -5.3156 & 0.8785\end{array}$

$\begin{array}{llll}\mathrm{H} & 2.2573 & -5.4302 & 0.8785\end{array}$

$\begin{array}{llll}\mathrm{H} & 2.2573 & -5.4302 & -0.8785\end{array}$

$\begin{array}{llll}\mathrm{H} & 3.3686 & -3.1674 & -0.8785\end{array}$

$\begin{array}{llll}\mathrm{H} & 3.3686 & -3.1674 & 0.8785\end{array}$

$\begin{array}{llll}\mathrm{H} & 0.8501 & -3.2808 & 0.8785\end{array}$

$\begin{array}{llll}\mathrm{H} & 0.8501 & -3.2808 & -0.8785\end{array}$

$\begin{array}{llll}\mathrm{H} & 1.9623 & -1.0184 & -0.8785\end{array}$

$\begin{array}{llll}\mathrm{H} & 1.9623 & -1.0184 & 0.8785\end{array}$

$\begin{array}{llll}\mathrm{H} & -0.5563 & -1.1311 & 0.8785\end{array}$

$\begin{array}{llll}\mathrm{H} & -0.5563 & -1.1311 & -0.8785\end{array}$

$\begin{array}{llll}\mathrm{H} & 0.5563 & 1.1311 & -0.8785\end{array}$

$\begin{array}{llll}\mathrm{H} & 0.5563 & 1.1311 & 0.8785\end{array}$

$\begin{array}{llll}\mathrm{H} & -1.9623 & 1.0184 & 0.8785\end{array}$ 


\begin{tabular}{lrrr}
$\mathrm{H}$ & -1.9623 & 1.0184 & -0.8785 \\
$\mathrm{H}$ & -0.8501 & 3.2808 & -0.8785 \\
$\mathrm{H}$ & -0.8501 & 3.2808 & 0.8785 \\
$\mathrm{H}$ & -3.3686 & 3.1674 & 0.8785 \\
$\mathrm{H}$ & -3.3686 & 3.1674 & -0.8785 \\
$\mathrm{H}$ & -2.2573 & 5.4302 & -0.8785 \\
$\mathrm{H}$ & -2.2573 & 5.4302 & 0.8785 \\
$\mathrm{H}$ & -4.7757 & 5.3156 & 0.8785 \\
$\mathrm{H}$ & -4.7757 & 5.3156 & -0.8785 \\
$\mathrm{H}$ & -3.6656 & 7.5790 & -0.8786 \\
$\mathrm{H}$ & -3.6656 & 7.5790 & 0.8786 \\
$\mathrm{H}$ & -6.1843 & 7.4642 & 0.8786 \\
$\mathrm{H}$ & -6.1843 & 7.4642 & -0.8786 \\
$\mathrm{H}$ & 5.0755 & -9.7261 & -0.8783 \\
$\mathrm{H}$ & 5.0755 & -9.7261 & 0.8783 \\
$\mathrm{H}$ & 7.0073 & -11.0634 & 0.0000 \\
$\mathrm{H}$ & 7.6076 & -9.6498 & 0.8854 \\
$\mathrm{H}$ & 7.6076 & -9.6498 & -0.8854 \\
$\mathrm{H}$ & -5.0755 & 9.7261 & -0.8783 \\
$\mathrm{H}$ & -5.0755 & 9.7261 & 0.8783 \\
$\mathrm{H}$ & -7.0073 & 11.0634 & 0.0000 \\
$\mathrm{H}$ & -7.6076 & 9.6498 & 0.8854 \\
$\mathrm{H}$ & -7.6076 & 9.6498 & -0.8854 \\
\hline
\end{tabular}

11chain0

$\begin{array}{rrrr} \\ \mathrm{C} & -13.4799 & -0.3607 & -0.0004 \\ \mathrm{C} & -12.1987 & 0.4808 & -0.0002 \\ \mathrm{C} & -10.9179 & -0.3645 & -0.0003 \\ \mathrm{C} & -9.6298 & 0.4697 & 0.0000 \\ \mathrm{C} & -8.3495 & -0.3765 & -0.0001 \\ \mathrm{C} & -7.0612 & 0.4575 & 0.0001 \\ \mathrm{C} & -5.7812 & -0.3892 & 0.0001 \\ \mathrm{C} & -4.4927 & 0.4445 & 0.0002 \\ \mathrm{C} & -3.2128 & -0.4025 & 0.0002 \\ \mathrm{C} & -1.9241 & 0.4309 & 0.0003 \\ \mathrm{C} & -0.6444 & -0.4163 & 0.0002 \\ \mathrm{C} & 0.6444 & 0.4169 & 0.0002 \\ \mathrm{C} & 1.9241 & -0.4303 & 0.0003 \\ \mathrm{C} & 3.2129 & 0.4030 & 0.0002 \\ \mathrm{C} & 4.4926 & -0.4441 & 0.0002 \\ \mathrm{C} & 5.7813 & 0.3894 & 0.0001 \\ \mathrm{C} & 7.0612 & -0.4574 & 0.0001 \\ \mathrm{C} & 8.3496 & 0.3765 & -0.0001 \\ \mathrm{C} & 9.6298 & -0.4699 & 0.0000 \\ \mathrm{C} & 10.9179 & 0.3640 & -0.0003 \\ \mathrm{C} & 12.1986 & -0.4815 & -0.0002 \\ \mathrm{C} & 13.4800 & 0.3598 & -0.0005 \\ \mathrm{H} & -14.3769 & 0.2718 & -0.0004 \\ \mathrm{H} & -13.5298 & -1.0092 & 0.8848 \\ \mathrm{H} & -13.5297 & -1.0089 & -0.8860 \\ \mathrm{H} & -12.1954 & 1.1439 & 0.8784 \\ \mathrm{H} & -12.1953 & 1.1443 & -0.8786 \\ & & \mathrm{~S} 21 & \\ & & & \end{array}$




\begin{tabular}{lrrr}
$\mathrm{H}$ & -10.9217 & -1.0288 & -0.8792 \\
$\mathrm{H}$ & -10.9218 & -1.0292 & 0.8784 \\
$\mathrm{H}$ & -9.6269 & 1.1338 & 0.8789 \\
$\mathrm{H}$ & -9.6268 & 1.1341 & -0.8787 \\
$\mathrm{H}$ & -8.3526 & -1.0407 & -0.8789 \\
$\mathrm{H}$ & -8.3527 & -1.0410 & 0.8785 \\
$\mathrm{H}$ & -7.0580 & 1.1217 & 0.8790 \\
$\mathrm{H}$ & -7.0579 & 1.1220 & -0.8785 \\
$\mathrm{H}$ & -5.7845 & -1.0534 & -0.8788 \\
$\mathrm{H}$ & -5.7846 & -1.0536 & 0.8787 \\
$\mathrm{H}$ & -4.4892 & 1.1087 & 0.8790 \\
$\mathrm{H}$ & -4.4892 & 1.1089 & -0.8785 \\
$\mathrm{H}$ & -3.2163 & -1.0668 & -0.8786 \\
$\mathrm{H}$ & -3.2164 & -1.0669 & 0.8789 \\
$\mathrm{H}$ & -1.9206 & 1.0951 & 0.8790 \\
$\mathrm{H}$ & -1.9205 & 1.0952 & -0.8785 \\
$\mathrm{H}$ & -0.6480 & -1.0807 & -0.8785 \\
$\mathrm{H}$ & -0.6480 & -1.0807 & 0.8790 \\
$\mathrm{H}$ & 0.6480 & 1.0813 & 0.8790 \\
$\mathrm{H}$ & 0.6480 & 1.0813 & -0.8785 \\
$\mathrm{H}$ & 1.9205 & -1.0947 & -0.8784 \\
$\mathrm{H}$ & 1.9205 & -1.0946 & 0.8790 \\
$\mathrm{H}$ & 3.2164 & 1.0674 & 0.8789 \\
$\mathrm{H}$ & 3.2164 & 1.0673 & -0.8786 \\
$\mathrm{H}$ & 4.4891 & -1.1085 & -0.8784 \\
$\mathrm{H}$ & 4.4892 & -1.1083 & 0.8790 \\
$\mathrm{H}$ & 5.7847 & 1.0539 & 0.8787 \\
$\mathrm{H}$ & 5.7847 & 1.0537 & -0.8787 \\
$\mathrm{H}$ & 7.0578 & -1.1219 & -0.8785 \\
$\mathrm{H}$ & 7.0579 & -1.1216 & 0.8790 \\
$\mathrm{H}$ & 8.3529 & 1.0410 & 0.8786 \\
$\mathrm{H}$ & 8.3528 & 1.0407 & -0.8789 \\
$\mathrm{H}$ & 9.6266 & -1.1344 & -0.8787 \\
$\mathrm{H}$ & 9.6267 & -1.1341 & 0.8789 \\
$\mathrm{H}$ & 10.9220 & 1.0287 & 0.8784 \\
$\mathrm{H}$ & 10.9219 & 1.0284 & -0.8792 \\
$\mathrm{H}$ & 12.1951 & -1.1449 & -0.8786 \\
$\mathrm{H}$ & 12.1952 & -1.1446 & 0.8784 \\
$\mathrm{H}$ & 14.3768 & -0.2729 & -0.0004 \\
$\mathrm{H}$ & 13.5300 & 1.0083 & 0.8848 \\
$\mathrm{H}$ & 13.5299 & 1.0079 & -0.8860 \\
& & & \\
\hline & & 11 &
\end{tabular}

11chain1

\begin{tabular}{rrrr} 
& \\
$\mathrm{C}$ & -3.0611 & 0.5872 & 0.0000 \\
$\mathrm{C}$ & -1.7231 & -0.1558 & -0.0006 \\
$\mathrm{C}$ & -0.5243 & 0.7756 & -0.0002 \\
$\mathrm{O}$ & 0.6318 & 0.0731 & -0.0013 \\
$\mathrm{C}$ & 1.8465 & 0.8562 & -0.0009 \\
$\mathrm{C}$ & 3.0239 & -0.1076 & -0.0007 \\
$\mathrm{O}$ & -0.5701 & 1.9841 & 0.0011 \\
\multicolumn{4}{c}{$\mathrm{S} 22$}
\end{tabular}




\begin{tabular}{|c|c|c|c|}
\hline & 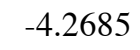 & -0 & \\
\hline & -5.6140 & 0.3792 & 0.0001 \\
\hline & 4.3725 & 0.6256 & -0.0004 \\
\hline & 5.5780 & -0.3242 & -0.0003 \\
\hline & -6.8291 & -0.5579 & -0.000 \\
\hline & -8.1735 & 0.1824 & 0.000 \\
\hline & 6.9282 & .4050 & 0.000 \\
\hline & 8.1369 & -0.5405 & 0.0001 \\
\hline & -9.3903 & -0.7527 & 0.000 \\
\hline & -10.7343 & -0.0120 & 0.000 \\
\hline & 9.4864 & US & 000 \\
\hline & 10.6968 & -0 & 0.000 \\
\hline & -11.9516 & -0.9463 & 0.000 \\
\hline & -13.2899 & -0.1 & 0.000 \\
\hline & 12.0458 & -0.0212 & $0.000 t$ \\
\hline & 13.2497 & -0.9 & $0.000 t$ \\
\hline & -3.1044 & & -0.874 \\
\hline & -3.1041 & 1.2497 & 0.8753 \\
\hline & -1.6346 & -0.8 & -0.8747 \\
\hline & -1.6344 & -0.8184 & 0.8727 \\
\hline & 1.8495 & 1.5068 & 0.882 \\
\hline & 1.8500 & & -0.884 \\
\hline & 2.9507 & -0. & -0.8814 \\
\hline & 2.9504 & -0 & 0.880 \\
\hline & -4.2168 & -1.0217 & 0.878 \\
\hline & -4.2170 & -1.0209 & -0.879 \\
\hline F & -5.6644 & 1. & -0.8782 \\
\hline $\mathrm{H}$ & -5.6 & & 0.878 \\
\hline $\mathrm{H}$ & 4.4306 & 64 & 0.8786 \\
\hline & $4 .{ }^{2}$ & & -0.879 \\
\hline & 5.5202 & -0.9 & -0.8792 \\
\hline & 5.5200 & -0.9853 & 0.878 \\
\hline $\mathrm{H}$ & -6.7784 & -1 . & 0.878 \\
\hline $\mathrm{H}$ & -6.7786 & -1 & -0.8791 \\
\hline & -8.2237 & & $-0.878 ?$ \\
\hline & -8.2235 & 46 & 0.879 \\
\hline & 6.9838 & 1.0668 & 0.878 \\
\hline $\mathrm{H}$ & 6.9840 & 3 & -0.878 \\
\hline $\mathrm{H}$ & 16 & -1 & -0.878 \\
\hline $\mathrm{H}$ & 8.0813 & -1 & 0.878 \\
\hline & -9.3403 & -1.4 & 0.878 \\
\hline & -9.3404 & -1.4 & $-0.878 \varepsilon$ \\
\hline f & 0.7854 & 0.6507 & -0.878 \\
\hline $\mathrm{H}$ & -10.7852 & 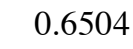 & 0.879 \\
\hline 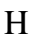 & 9.5410 & 0.8523 & 0.879 \\
\hline & 9.5412 & 0.8525 & -0.878 \\
\hline & 10.6434 & -1.4150 & -0.878 \\
\hline $\mathrm{F}$ & 10.6431 & -1.4 & 0.879 \\
\hline $\mathrm{H}$ & -11.9012 & -1 & 0.878 \\
\hline $\mathrm{H}$ & -11.9013 & -1.6075 & -0.878 \\
\hline $\mathrm{H}$ & -14.1389 & -0.8942 & 0.000 \\
\hline $\mathrm{H}$ & -13.3865 & 0.4444 & -0.884 \\
\hline & -13.3863 & & 0.886 \\
\hline & 12.0995 & 0.6399 & 0.879 \\
\hline $\mathrm{H}$ & 12.0997 & 0.6400 & -0.877 \\
\hline
\end{tabular}




$\begin{array}{lrrr}\mathrm{H} & 14.1977 & -0.4169 & 0.0008 \\ \mathrm{H} & 13.2438 & -1.6201 & -0.8848 \\ \mathrm{H} & 13.2436 & -1.6203 & 0.8860\end{array}$

12chain 0

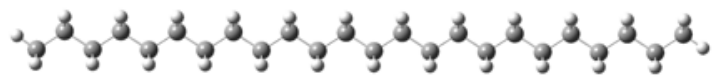

$\begin{array}{llll}\text { C } & 7.0329 & -9.9780 & 0.0000\end{array}$

$\begin{array}{llll}\text { C } & 5.6264 & -9.3644 & 0.0000\end{array}$

$\begin{array}{llll}\text { C } & 5.6254 & -7.8296 & 0.0000\end{array}$

$\begin{array}{llll}\text { C } & 4.2188 & -7.2157 & 0.0000\end{array}$

$\begin{array}{llll}\text { C } & 4.2183 & -5.6810 & 0.0000\end{array}$

$\begin{array}{llll}\text { C } & 2.8119 & -5.0666 & 0.0000\end{array}$

$\begin{array}{llll}\text { C } & 2.8119 & -3.5318 & 0.0000\end{array}$

$\begin{array}{llll}\text { C } & 1.4057 & -2.9171 & 0.0000\end{array}$

$\begin{array}{llll}\text { C } & 1.4060 & -1.3823 & 0.0000\end{array}$

$\begin{array}{llll}\text { C } & -0.0002 & -0.7674 & 0.0000\end{array}$

$\begin{array}{llll}\text { C } & 0.0002 & 0.7674 & 0.0000\end{array}$

$\begin{array}{llll}\text { C } & -1.4060 & 1.3823 & 0.0000\end{array}$

$\begin{array}{llll}\text { C } & -1.4057 & 2.9171 & 0.0000\end{array}$

$\begin{array}{llll}\text { C } & -2.8119 & 3.5318 & 0.0000\end{array}$

$\begin{array}{llll}\text { C } & -2.8119 & 5.0666 & 0.0000\end{array}$

$\begin{array}{llll}\text { C } & -4.2183 & 5.6810 & 0.0000\end{array}$

$\begin{array}{llll}\text { C } & 7.0349 & -11.5126 & 0.0000\end{array}$

$\begin{array}{llll}\text { C } & 8.4439 & -12.1166 & 0.0000\end{array}$

$\begin{array}{llll}\text { C } & -4.2188 & 7.2157 & 0.0000\end{array}$

$\begin{array}{llll}\text { C } & -5.6254 & 7.8296 & 0.0000\end{array}$

$\begin{array}{llll}\text { C } & -5.6264 & 9.3644 & 0.0000\end{array}$

$\begin{array}{llll}\text { C } & -7.0329 & 9.9780 & 0.0000\end{array}$

$\begin{array}{llll}\text { C } & -7.0349 & 11.5126 & 0.0000\end{array}$

$\begin{array}{llll}\text { C } & -8.4439 & 12.1166 & 0.0000\end{array}$

$\begin{array}{llll}\mathrm{H} & 7.5892 & -9.6145 & 0.8788\end{array}$

$\begin{array}{llll}\mathrm{H} & 7.5892 & -9.6145 & -0.8788\end{array}$

$\begin{array}{llll}\mathrm{H} & 5.0708 & -9.7285 & 0.8788\end{array}$

$\begin{array}{llll}\mathrm{H} & 5.0708 & -9.7285 & -0.8788\end{array}$

$\begin{array}{llll}\mathrm{H} & 6.1811 & -7.4656 & -0.8787\end{array}$

$\begin{array}{llll}\mathrm{H} & 6.1811 & -7.4656 & 0.8787\end{array}$

$\begin{array}{llll}\mathrm{H} & 3.6630 & -7.5797 & 0.8787\end{array}$

$\begin{array}{llll}\mathrm{H} & 3.6630 & -7.5797 & -0.8787\end{array}$

$\begin{array}{llll}\mathrm{H} & 4.7742 & -5.3171 & -0.8787\end{array}$

$\begin{array}{llll}\mathrm{H} & 4.7742 & -5.3171 & 0.8787\end{array}$

$\begin{array}{llll}\mathrm{H} & 2.2561 & -5.4303 & 0.8787\end{array}$

$\begin{array}{llll}\mathrm{H} & 2.2561 & -5.4303 & -0.8787\end{array}$

$\begin{array}{llll}\mathrm{H} & 3.3679 & -3.1681 & -0.8787\end{array}$

$\begin{array}{llll}\mathrm{H} & 3.3679 & -3.1681 & 0.8787\end{array}$

$\begin{array}{llll}\mathrm{H} & 0.8497 & -3.2807 & 0.8787\end{array}$

$\begin{array}{llll}\mathrm{H} & 0.8497 & -3.2807 & -0.8787\end{array}$

$\begin{array}{llll}\mathrm{H} & 1.9620 & -1.0187 & -0.8787\end{array}$

$\begin{array}{llll}\mathrm{H} & 1.9620 & -1.0187 & 0.8787\end{array}$

$\begin{array}{llll}\mathrm{H} & -0.5562 & -1.1310 & 0.8787\end{array}$

$\begin{array}{llll}\mathrm{H} & -0.5562 & -1.1310 & -0.8787\end{array}$

$\begin{array}{llll}\mathrm{H} & 0.5562 & 1.1310 & -0.8787\end{array}$

$\begin{array}{llll}\mathrm{H} & 0.5562 & 1.1310 & 0.8787\end{array}$ 


$\begin{array}{lrrr} & & & \\ \mathrm{H} & -1.9620 & 1.0187 & 0.8787 \\ \mathrm{H} & -1.9620 & 1.0187 & -0.8787 \\ \mathrm{H} & -0.8497 & 3.2807 & -0.8787 \\ \mathrm{H} & -3.3679 & 3.2807 & 0.8787 \\ \mathrm{H} & -3.3679 & 3.1681 & 0.8787 \\ \mathrm{H} & -2.2561 & 5.4303 & -0.8787 \\ \mathrm{H} & -2.2561 & 5.4303 & 0.8787 \\ \mathrm{H} & -4.7742 & 5.3171 & 0.8787 \\ \mathrm{H} & -4.7742 & 5.3171 & -0.8787 \\ \mathrm{H} & 6.4800 & -11.8759 & -0.8785 \\ \mathrm{H} & 6.4800 & -11.8759 & 0.8785 \\ \mathrm{H} & 8.4114 & -13.2137 & 0.0000 \\ \mathrm{H} & 9.0120 & -11.8004 & 0.8854 \\ \mathrm{H} & 9.0120 & -11.8004 & -0.8854 \\ \mathrm{H} & -3.6630 & 7.5797 & -0.8787 \\ \mathrm{H} & -3.6630 & 7.5797 & 0.8787 \\ \mathrm{H} & -6.1811 & 7.4656 & 0.8787 \\ \mathrm{H} & -6.1811 & 7.4656 & -0.8787 \\ \mathrm{H} & -5.0708 & 9.7285 & -0.8788 \\ \mathrm{H} & -5.0708 & 9.7285 & 0.8788 \\ \mathrm{H} & -7.5892 & 9.6145 & 0.8788 \\ \mathrm{H} & -7.5892 & 9.6145 & -0.8788 \\ \mathrm{H} & -6.4800 & 11.8759 & -0.8785 \\ \mathrm{H} & -6.4800 & 11.8759 & 0.8785 \\ \mathrm{H} & -8.4114 & 13.2137 & 0.0000 \\ \mathrm{H} & -9.0120 & 11.8004 & 0.8854 \\ \mathrm{H} & -9.0120 & 11.8004 & -0.8854 \\ & & & \end{array}$

13chain0

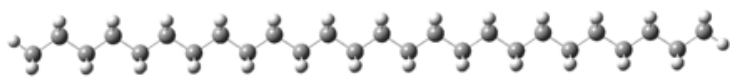

$\begin{array}{lrrr}\text { C } & -16.0478 & -0.3717 & 0.0001 \\ \text { C } & -14.7679 & 0.4721 & -0.0003 \\ \text { C } & -13.4857 & -0.3711 & 0.0001 \\ \text { C } & -12.1991 & 0.4651 & -0.0002 \\ \text { C } & -10.9174 & -0.3790 & 0.0001 \\ \text { C } & -9.6304 & 0.4572 & -0.0002 \\ \text { C } & -8.3491 & -0.3875 & 0.0001 \\ \text { C } & -7.0618 & 0.4482 & -0.0001 \\ \text { C } & -5.7807 & -0.3969 & 0.0001 \\ \text { C } & -4.4932 & 0.4384 & 0.0000 \\ \text { C } & -3.2123 & -0.4070 & 0.0001 \\ \text { C } & -1.9247 & 0.4281 & 0.0000 \\ \text { C } & -0.6439 & -0.4174 & 0.0000 \\ \text { C } & 0.6438 & 0.4176 & 0.0001 \\ \text { C } & 1.9246 & -0.4279 & 0.0000 \\ \text { C } & 3.2123 & 0.4071 & 0.0001 \\ \text { C } & 4.4932 & -0.4382 & -0.0001 \\ \text { C } & 5.7807 & 0.3970 & 0.0001 \\ \text { C } & 7.0618 & -0.4481 & -0.0001 \\ \text { C } & 8.3491 & 0.3875 & 0.0001 \\ \text { C } & 9.6304 & -0.4572 & -0.0002\end{array}$




\begin{tabular}{|c|c|c|c|}
\hline & & & \\
\hline & 12.1991 & -0.4653 & -0.0002 \\
\hline & 13.4857 & 0.3710 & 0.0001 \\
\hline & 14.7679 & -0.4723 & -0.0002 \\
\hline & 16.0478 & .3713 & 0.000 \\
\hline & -16.9458 & 0.2594 & -0.000 \\
\hline & -16.0965 & -1.0196 & 0.885 \\
\hline & -16.0965 & -1.0204 & -0.8850 \\
\hline & -14.7657 & 1.1357 & 0.877 \\
\hline & -14.7657 & 1.1349 & -0.8790 \\
\hline & -13.4885 & -1.0360 & -0.878 \\
\hline & -13.4886 & -1.0353 & 0.879 \\
\hline & -12.1972 & 1.1298 & 0.878 \\
\hline & -12.1971 & 1.1291 & -0.879 \\
\hline & -10.9194 & -1.0437 & -0.878 \\
\hline & -10.9195 & -1. & .879 \\
\hline & -9.6 & 1.1218 & .878 \\
\hline & -9.6282 & 1.1212 & -0.879 \\
\hline & -8.3514 & -1. & -0.878 \\
\hline & -8.3514 & -1.0516 & 0.879 \\
\hline & -7.0594 & 1 & 0.878 \\
\hline F & -7.0 & & -0.879 \\
\hline & -5 . & -1. & -0.878 \\
\hline & -5 . & -1 & 0.879 \\
\hline & -4.4906 & 29 & 0.878 \\
\hline & -4.4906 & 26 & -0.878 \\
\hline F & -3.2 & -1 . & -0.878 \\
\hline $\mathrm{H}$ & -3. & -1 & 7 \\
\hline $\mathrm{H}$ & -1.9 & 25 & $.878^{\prime}$ \\
\hline & -1.9220 & & -0.878 \\
\hline & -0.6466 & -1.0 & $-0.878^{\prime}$ \\
\hline & -0.6466 & -1.0 & 0.878 \\
\hline H & & & 78 \\
\hline $\mathrm{H}$ & 55 & & $-0.878 c$ \\
\hline & 1.9220 & -1.0922 & -0.8788 \\
\hline & 1.9219 & -1.0 & $0.878^{7}$ \\
\hline & 3.2150 & & 878 \\
\hline & 3.21 & 16 & -0.878 \\
\hline $\mathrm{H}$ & 06 & -1 . & -0.878 \\
\hline $\mathrm{H}$ & 06 & -1 . & 0.878 \\
\hline & & & 0.879 \\
\hline & 32 & & 0.878 \\
\hline & 7.0593 & -1.1122 & -0.879 \\
\hline $\mathrm{H}$ & 4 & -1 & 0.878 \\
\hline F & 8.3514 & 1.0516 & 0.879 \\
\hline & 8.3514 & 1.0521 & -0.878 \\
\hline & 9.6282 & -1.1212 & -0.879 \\
\hline & 82 & -1.1218 & 0.878 \\
\hline & 10. & 30 & 0.879 \\
\hline & 10.9195 & 1.0435 & -0.878 \\
\hline $\mathrm{H}$ & 12.1971 & & -0.879 \\
\hline $\mathrm{H}$ & 12.1971 & -1.1299 & 0.878 \\
\hline & 13. & & 792 \\
\hline F & 13.4886 & 1.0358 & -0.878 \\
\hline & 14.7657 & -1.1352 & -0.879 \\
\hline
\end{tabular}




$\begin{array}{rrrr}\mathrm{H} & 14.7657 & -1.1359 & 0.8780 \\ \mathrm{H} & 16.9458 & -0.2598 & -0.0001 \\ \mathrm{H} & 16.0966 & 1.0193 & 0.8858 \\ \mathrm{H} & 16.0966 & 1.0201 & -0.8850\end{array}$

13chain 1

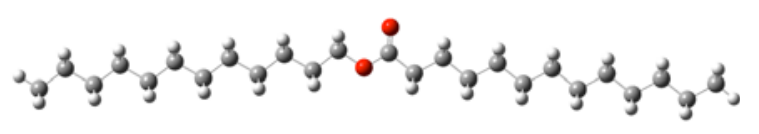

$\begin{array}{llll}\text { C } & -3.0628 & 0.6915 & 0.0000\end{array}$

$\begin{array}{llll}\text { C } & -1.7252 & -0.0524 & -0.0005\end{array}$

$\begin{array}{llll}\text { C } & -0.5260 & 0.8785 & -0.0003\end{array}$

$\begin{array}{llll}\text { O } & 0.6299 & 0.1757 & -0.0004\end{array}$

$\begin{array}{llll}\text { C } & 1.8447 & 0.9587 & -0.0002\end{array}$

$\begin{array}{llll}\text { C } & 3.0221 & -0.0050 & 0.0000\end{array}$

$\begin{array}{llll}\mathrm{O} & -0.5715 & 2.0870 & 0.0000\end{array}$

$\begin{array}{llll}\text { C } & -4.2710 & -0.2536 & -0.0004\end{array}$

$\begin{array}{llll}\text { C } & -5.6157 & 0.4859 & 0.0000\end{array}$

$\begin{array}{llll}\text { C } & 4.3705 & 0.7286 & 0.0001\end{array}$

$\begin{array}{llll}\text { C } & 5.5765 & -0.2205 & 0.0002\end{array}$

$\begin{array}{llll}\text { C } & -6.8321 & -0.4495 & -0.0003\end{array}$

$\begin{array}{llll}\text { C } & -8.1753 & 0.2930 & 0.0000\end{array}$

$\begin{array}{llll}\text { C } & 6.9261 & 0.5099 & 0.0003\end{array}$

$\begin{array}{llll}\text { C } & 8.1359 & -0.4343 & 0.0003\end{array}$

$\begin{array}{llll}\text { C } & -9.3940 & -0.6397 & -0.0002\end{array}$

$\begin{array}{llll}\text { C } & -10.7362 & 0.1046 & 0.0001\end{array}$

$\begin{array}{llll}\text { C } & 9.4843 & 0.2985 & 0.0003\end{array}$

$\begin{array}{llll}\text { C } & 10.6963 & -0.6428 & 0.0002\end{array}$

$\begin{array}{llll}\text { C } & -11.9558 & -0.8268 & 0.0000\end{array}$

$\begin{array}{llll}\text { C } & -13.2977 & -0.0824 & 0.0002\end{array}$

$\begin{array}{llll}\text { C } & 12.0437 & 0.0919 & 0.0001\end{array}$

$\begin{array}{llll}\text { C } & 13.2569 & -0.8475 & 0.0000\end{array}$

$\begin{array}{llll}\text { C } & -14.5176 & -1.0133 & 0.0002\end{array}$

$\begin{array}{llll}\text { C } & -15.8538 & -0.2621 & 0.0004\end{array}$

$\begin{array}{llll}\text { C } & 14.6039 & -0.1122 & 0.0000\end{array}$

$\begin{array}{llll}\text { C } & 15.8105 & -1.0575 & -0.0002\end{array}$

$\begin{array}{llll}\mathrm{H} & -3.1056 & 1.3548 & -0.8747\end{array}$

$\begin{array}{llll}\mathrm{H} & -3.1055 & 1.3540 & 0.8753\end{array}$

$\begin{array}{llll}\mathrm{H} & -1.6371 & -0.7141 & -0.8746\end{array}$

$\begin{array}{llll}\mathrm{H} & -1.6368 & -0.7149 & 0.8729\end{array}$

$\begin{array}{llll}\mathrm{H} & 1.8478 & 1.6094 & 0.8835\end{array}$

$\begin{array}{llll}\mathrm{H} & 1.8481 & 1.6093 & -0.8840\end{array}$

$\begin{array}{llll}\mathrm{H} & 2.9491 & -0.6588 & -0.8807\end{array}$

$\begin{array}{llll}\mathrm{H} & 2.9489 & -0.6587 & 0.8808\end{array}$

$\begin{array}{llll}\mathrm{H} & -4.2200 & -0.9165 & 0.8782\end{array}$

$\begin{array}{llll}\mathrm{H} & -4.2201 & -0.9158 & -0.8795\end{array}$

$\begin{array}{llll}\mathrm{H} & -4.2201 & -0.9158 & -0.8795 \\ \mathrm{H} & -5.6652 & 1.1486 & -0.8783\end{array}$

\begin{tabular}{llll}
$\mathrm{H}$ & -5.6652 & 1.1486 & -0.8783 \\
\hline
\end{tabular}

$\begin{array}{llll}\mathrm{H} & -5.6651 & 1.1480 & 0.8788\end{array}$

$\begin{array}{llll}\mathrm{H} & 4.4283 & 1.3895 & 0.8791\end{array}$

$\begin{array}{llll}\mathrm{H} & 4.4285 & 1.3895 & -0.8789\end{array}$

$\begin{array}{llll}\mathrm{H} & 5.5192 & -0.8816 & -0.8787\end{array}$

$\begin{array}{llll}\mathrm{H} & 5.5191 & -0.8816 & 0.8791\end{array}$

$\begin{array}{llll}\mathrm{H} & -6.7825 & -1.1122 & 0.8784\end{array}$

$\begin{array}{llll}\mathrm{H} & -6.7825 & -1.1116 & -0.8793\end{array}$ 


$\begin{array}{rrrr}\mathrm{H} & -8.2242 & 0.9558 & -0.8785 \\ \mathrm{H} & -8.2241 & 0.9553 & 0.8789 \\ \mathrm{H} & 6.9812 & 1.1717 & 0.8790 \\ \mathrm{H} & 6.9812 & 1.1718 & -0.8785 \\ \mathrm{H} & 8.0813 & -1.0962 & -0.8786 \\ \mathrm{H} & 8.0813 & -1.0962 & 0.8791 \\ \mathrm{H} & -9.3454 & -1.3024 & 0.8785 \\ \mathrm{H} & -9.3454 & -1.3020 & -0.8791 \\ \mathrm{H} & -10.7844 & 0.7674 & -0.8785 \\ \mathrm{H} & -10.7843 & 0.7670 & 0.8789 \\ \mathrm{H} & 9.5381 & 0.9607 & 0.8790 \\ \mathrm{H} & 9.5380 & 0.9607 & -0.8784 \\ \mathrm{H} & 10.6429 & -1.3049 & -0.8786 \\ \mathrm{H} & 10.6430 & -1.3050 & 0.8789 \\ \mathrm{H} & -11.9076 & -1.4896 & 0.8786 \\ \mathrm{H} & -11.9077 & -1.4892 & -0.8790 \\ \mathrm{H} & -13.3470 & 0.5805 & -0.8784 \\ \mathrm{H} & -13.3468 & 0.5801 & 0.8791 \\ \mathrm{H} & 12.0966 & 0.7542 & 0.8789 \\ \mathrm{H} & 12.0965 & 0.7543 & -0.8786 \\ \mathrm{H} & 13.2053 & -1.5099 & -0.8788 \\ \mathrm{H} & 13.2054 & -1.5100 & 0.8788 \\ \mathrm{H} & -14.4689 & -1.6751 & 0.8785 \\ \mathrm{H} & -14.4691 & -1.6747 & -0.8785 \\ \mathrm{H} & -16.7048 & -0.9553 & 0.0004 \\ \mathrm{H} & -15.9487 & 0.3813 & -0.8848 \\ \mathrm{H} & -15.9485 & 0.3810 & 0.8860 \\ \mathrm{H} & 14.6559 & 0.5491 & 0.8785 \\ \mathrm{H} & 14.6558 & 0.5492 & -0.8784 \\ \mathrm{H} & 16.7570 & -0.5018 & -0.0002 \\ \mathrm{H} & 15.8063 & -1.7077 & -0.8856 \\ \mathrm{H} & 15.8064 & -1.7078 & 0.8852\end{array}$

14chain0

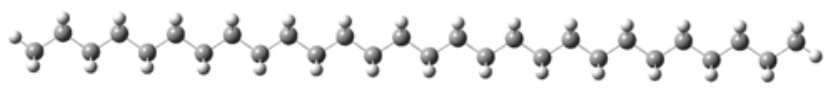

$\begin{array}{lrrr}\mathrm{C} & 0.4245 & -14.7683 & 0.0000 \\ \mathrm{C} & -0.4171 & -13.4852 & 0.0000 \\ \mathrm{C} & 0.4217 & -12.2000 & 0.0000 \\ \mathrm{C} & -0.4197 & -10.9165 & 0.0000 \\ \mathrm{C} & 0.4197 & -9.6317 & 0.0000 \\ \mathrm{C} & -0.4211 & -8.3478 & 0.0000 \\ \mathrm{C} & 0.4189 & -7.0634 & 0.0000 \\ \mathrm{C} & -0.4214 & -5.7791 & 0.0000 \\ \mathrm{C} & 0.4190 & -4.4950 & 0.0000 \\ \mathrm{C} & -0.4211 & -3.2105 & 0.0000 \\ \mathrm{C} & 0.4195 & -1.9265 & 0.0000 \\ \mathrm{C} & -0.4203 & -0.6420 & 0.0000 \\ \mathrm{C} & 0.4203 & 0.6420 & 0.0000 \\ \mathrm{C} & -0.4195 & 1.9265 & 0.0000 \\ \mathrm{C} & 0.4211 & 3.2105 & 0.0000 \\ \mathrm{C} & -0.4190 & 4.4950 & 0.0000\end{array}$




\begin{tabular}{|c|c|c|c|}
\hline C & -0.4132 & -16.0541 & 0.0000 \\
\hline$C$ & 0.4359 & -17.3304 & 0.0000 \\
\hline $\mathrm{C}$ & 0.4214 & 5.7791 & 0.0000 \\
\hline $\mathrm{C}$ & -0.4189 & 7.0634 & 0.0000 \\
\hline $\mathrm{C}$ & 0.4211 & 8.3478 & 0.0000 \\
\hline $\mathrm{C}$ & -0.4197 & 9.6317 & 0.0000 \\
\hline $\mathrm{C}$ & 0.4197 & 10.9165 & 0.0000 \\
\hline $\mathrm{C}$ & -0.4217 & 12.2000 & 0.0000 \\
\hline C & 0.4171 & 13.4852 & 0.0000 \\
\hline $\mathrm{C}$ & -0.4245 & 14.7683 & 0.0000 \\
\hline $\mathrm{C}$ & 0.4132 & 16.0541 & 0.0000 \\
\hline $\mathrm{C}$ & -0.4359 & 17.3304 & 0.0000 \\
\hline $\mathrm{H}$ & 1.0891 & -14.7684 & 0.8788 \\
\hline $\mathrm{H}$ & 1.0891 & -14.7684 & -0.8788 \\
\hline $\mathrm{H}$ & -1.0815 & -13.4860 & 0.8788 \\
\hline $\mathrm{H}$ & -1.0815 & -13.4860 & -0.8788 \\
\hline $\mathrm{H}$ & 1.0860 & -12.1993 & -0.8787 \\
\hline $\mathrm{H}$ & 1.0860 & -12.1993 & 0.8787 \\
\hline $\mathrm{H}$ & -1.0840 & -10.9170 & 0.8787 \\
\hline $\mathrm{H}$ & -1.0840 & -10.9170 & -0.8787 \\
\hline $\mathrm{H}$ & 1.0841 & -9.6314 & -0.8787 \\
\hline $\mathrm{H}$ & 1.0841 & -9.6314 & 0.8787 \\
\hline $\mathrm{H}$ & -1.0854 & -8.3480 & 0.8787 \\
\hline $\mathrm{H}$ & -1.0854 & -8.3480 & -0.8787 \\
\hline $\mathrm{H}$ & 1.0833 & -7.0633 & -0.8787 \\
\hline $\mathrm{H}$ & 1.0833 & -7.0633 & 0.8787 \\
\hline $\mathrm{H}$ & -1.0857 & -5.7791 & 0.8787 \\
\hline $\mathrm{H}$ & -1.0857 & -5.7791 & -0.8787 \\
\hline $\mathrm{H}$ & 1.0833 & -4.4951 & -0.8787 \\
\hline $\mathrm{H}$ & 1.0833 & -4.4951 & 0.8787 \\
\hline $\mathrm{H}$ & -1.0854 & -3.2104 & 0.8787 \\
\hline $\mathrm{H}$ & -1.0854 & -3.2104 & -0.8787 \\
\hline $\mathrm{H}$ & 1.0839 & -1.9267 & -0.8787 \\
\hline $\mathrm{H}$ & 1.0839 & -1.9267 & 0.8787 \\
\hline $\mathrm{H}$ & -1.0847 & -0.6418 & 0.8787 \\
\hline $\mathrm{H}$ & -1.0847 & -0.6418 & -0.8787 \\
\hline $\mathrm{H}$ & 1.0847 & 0.6418 & -0.8787 \\
\hline $\mathrm{H}$ & 1.0847 & 0.6418 & 0.8787 \\
\hline $\mathrm{H}$ & -1.0839 & 1.9267 & 0.8787 \\
\hline $\mathrm{H}$ & -1.0839 & 1.9267 & -0.8787 \\
\hline $\mathrm{H}$ & 1.0854 & 3.2104 & -0.8787 \\
\hline $\mathrm{H}$ & 1.0854 & 3.2104 & 0.8787 \\
\hline $\mathrm{H}$ & -1.0833 & 4.4951 & 0.8787 \\
\hline $\mathrm{H}$ & -1.0833 & 4.4951 & -0.8787 \\
\hline $\mathrm{H}$ & -1.0765 & -16.0546 & -0.8785 \\
\hline $\mathrm{H}$ & -1.0765 & -16.0546 & 0.8785 \\
\hline $\mathrm{H}$ & -0.1913 & -18.2311 & 0.0000 \\
\hline $\mathrm{H}$ & 1.0845 & -17.3763 & 0.8854 \\
\hline $\mathrm{H}$ & 1.0845 & -17.3763 & -0.8854 \\
\hline $\mathrm{H}$ & 1.0857 & 5.7791 & -0.8787 \\
\hline $\mathrm{H}$ & 1.0857 & 5.7791 & 0.8787 \\
\hline $\mathrm{H}$ & -1.0833 & 7.0633 & 0.8787 \\
\hline $\mathrm{H}$ & -1.0833 & 7.0633 & -0.8787 \\
\hline $\mathrm{H}$ & 1.0854 & 8.3480 & -0.8787 \\
\hline $\mathrm{H}$ & 1.0854 & 8.3480 & 0.8787 \\
\hline
\end{tabular}




$\begin{array}{rrrr}\mathrm{H} & -1.0841 & 9.6314 & 0.8787 \\ \mathrm{H} & -1.0841 & 9.6314 & -0.8787 \\ \mathrm{H} & 1.0840 & 10.9170 & -0.8787 \\ \mathrm{H} & 1.0840 & 10.9170 & 0.8787 \\ \mathrm{H} & -1.0860 & 12.1993 & 0.8787 \\ \mathrm{H} & -1.0860 & 12.1993 & -0.8787 \\ \mathrm{H} & 1.0815 & 13.4860 & -0.8788 \\ \mathrm{H} & 1.0815 & 13.4860 & 0.8788 \\ \mathrm{H} & -1.0891 & 14.7684 & 0.8788 \\ \mathrm{H} & -1.0891 & 14.7684 & -0.8788 \\ \mathrm{H} & 1.0765 & 16.0546 & -0.8785 \\ \mathrm{H} & 1.0765 & 16.0546 & 0.8785 \\ \mathrm{H} & 0.1913 & 18.2311 & 0.0000 \\ \mathrm{H} & -1.0845 & 17.3763 & 0.8854 \\ \mathrm{H} & -1.0845 & 17.3763 & -0.8854\end{array}$

15chain0

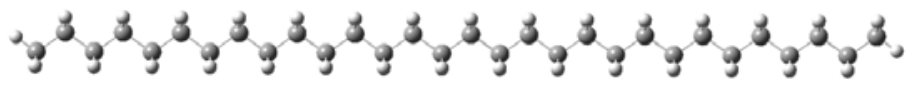

$\begin{array}{lrrr}\mathrm{C} & 18.6158 & -0.3819 & -0.0005 \\ \mathrm{C} & 17.3371 & 0.4636 & -0.0005 \\ \mathrm{C} & 16.0537 & -0.3778 & -0.0003 \\ \mathrm{C} & 14.7682 & 0.4602 & -0.0003 \\ \mathrm{C} & 13.4854 & -0.3822 & -0.0001 \\ \mathrm{C} & 12.1995 & 0.4556 & -0.0001 \\ \mathrm{C} & 10.9171 & -0.3874 & 0.0000 \\ \mathrm{C} & 9.6309 & 0.4498 & 0.0000 \\ \mathrm{C} & 8.3488 & -0.3937 & 0.0002 \\ \mathrm{C} & 7.0622 & 0.4430 & 0.0002 \\ \mathrm{C} & 5.7804 & -0.4010 & 0.0003 \\ \mathrm{C} & 4.4936 & 0.4354 & 0.0003 \\ \mathrm{C} & 3.2120 & -0.4089 & 0.0003 \\ \mathrm{C} & 1.9250 & 0.4273 & 0.0003 \\ \mathrm{C} & 0.6435 & -0.4172 & 0.0003 \\ \mathrm{C} & -0.6435 & 0.4188 & 0.0003 \\ \mathrm{C} & -1.9250 & -0.4257 & 0.0003 \\ \mathrm{C} & -3.2120 & 0.4104 & 0.0003 \\ \mathrm{C} & -4.4935 & -0.4341 & 0.0003 \\ \mathrm{C} & -5.7805 & 0.4021 & 0.0002 \\ \mathrm{C} & -7.0621 & -0.4421 & 0.0002 \\ \mathrm{C} & -8.3489 & 0.3944 & 0.0001 \\ \mathrm{C} & -9.6307 & -0.4495 & 0.0000 \\ \mathrm{C} & -10.9172 & 0.3874 & 0.0000 \\ \mathrm{C} & -12.1994 & -0.4560 & -0.0001 \\ \mathrm{C} & -13.4855 & 0.3814 & -0.0001 \\ \mathrm{C} & -14.7681 & -0.4614 & -0.0003 \\ \mathrm{C} & -16.0539 & 0.3761 & -0.0003 \\ \mathrm{C} & -17.3369 & -0.4658 & -0.0005 \\ \mathrm{C} & -18.6160 & 0.3792 & -0.0005 \\ \mathrm{H} & 19.5147 & 0.2480 & -0.0006 \\ \mathrm{H} & 18.6636 & -1.0303 & -0.8859 \\ \mathrm{H} & 18.6637 & -1.0302 & 0.8849 \\ \mathrm{H} & 17.3357 & 1.1268 & -0.8790 \\ & & \mathrm{~S} 30 & \\ & & & \end{array}$




\begin{tabular}{|c|c|c|c|}
\hline & & 69 & \\
\hline & 16.0557 & -1.0423 & . \\
\hline & 16.0556 & -1.0424 & -0.8791 \\
\hline & 14.7671 & 1.1245 & 0.8791 \\
\hline & 14.7672 & 1246 & 0.878 \\
\hline & 13.4866 & -1.0465 & 878 \\
\hline & 13.4865 & -1.0466 & $0.8 / 8$ \\
\hline & 12.1981 & 1.1199 & -0.878 \\
\hline & 12.1983 & 1.1200 & 0.8786 \\
\hline & 10.9187 & -1.0517 & 78 \\
\hline & 10.9 & -1.0518 & $0.878^{\prime}$ \\
\hline & & & \\
\hline & 9.6293 & 1.1 & 0. \\
\hline & 8.3506 & -1.0580 & 0.878 \\
\hline & 8.3505 & -1.0581 & -0.878 \\
\hline & & 73 & -0.878 \\
\hline & & & \\
\hline & & $-1 .($ & 790 \\
\hline & 23 & -1.0 & -0.878 \\
\hline & 15 & 1.0 & -0.878 \\
\hline & & 98 & 79 \\
\hline & & -1.0 & 79 \\
\hline & & -1. & \\
\hline & & & \\
\hline & 1.9229 & 16 & 0.879 \\
\hline & 0.6457 & -1.0 & 79 \\
\hline & & 15 & -0 \\
\hline & & & \\
\hline & & & \\
\hline & -1 . & -1.0 & 0.879 \\
\hline & -1.9228 & -1.0 & -0.878 \\
\hline F & -3.2141 & 47 & -0.878 \\
\hline & -3. & 47 & 79 \\
\hline & & 34 & \\
\hline & -4.4 & -1.0 & -0.878 \\
\hline & -5.7825 & 1.0665 & -0.878 \\
\hline F & -5.7826 & .0665 & 0.879 \\
\hline & -7. & 11 & 0 \\
\hline & -7 & -1 & $-0.878 c$ \\
\hline & & & -0.878 \\
\hline & & & 0.878 \\
\hline F & & 8 & $78^{\prime}$ \\
\hline H & -9.6 & -1.1 & $-0.878^{\prime}$ \\
\hline $\mathrm{H}$ & 08 & 8 & -0.8 \\
\hline $\mathrm{H}$ & -10 & 17 & \\
\hline & -12.1979 & -1.1204 & 0.878 \\
\hline & -12.1978 & -1.1203 & -0.878 \\
\hline & -13.4869 & 1.0458 & -0.878 \\
\hline $\mathrm{H}$ & -13.4 & & 0970 \\
\hline & -14.7668 & -1.1258 & 0.878 \\
\hline $\mathrm{H}$ & -14.7667 & -1.1257 & -0.879 \\
\hline & $-16 .($ & & -0.879 \\
\hline & & & \\
\hline & -17.3354 & -1.1291 & 0.878 \\
\hline & -17.3353 & -1.1290 & -0.879 \\
\hline
\end{tabular}




$\begin{array}{rrrr}\mathrm{H} & -19.5146 & -0.2510 & -0.0006 \\ \mathrm{H} & -18.6640 & 1.0277 & -0.8858 \\ \mathrm{H} & -18.6641 & 1.0275 & 0.8850\end{array}$

15chain 1

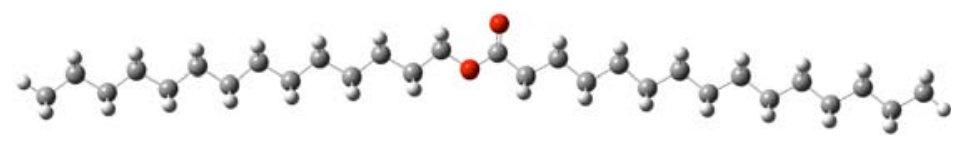

\begin{tabular}{lrrr}
$\mathrm{C}$ & -3.0641 & 0.7901 & 0.0002 \\
$\mathrm{C}$ & -1.7267 & 0.0460 & -0.0001 \\
$\mathrm{C}$ & -0.5274 & 0.9769 & -0.0001 \\
$\mathrm{O}$ & 0.6285 & 0.2741 & 0.0000 \\
$\mathrm{C}$ & 1.8433 & 1.0572 & -0.0001 \\
$\mathrm{C}$ & 3.0209 & 0.0937 & 0.0001 \\
$\mathrm{O}$ & -0.5728 & 2.1854 & -0.0004 \\
$\mathrm{C}$ & -4.2726 & -0.1546 & -0.0002 \\
$\mathrm{C}$ & -5.6169 & 0.5856 & 0.0002 \\
$\mathrm{C}$ & 4.3690 & 0.8278 & 0.0000 \\
$\mathrm{C}$ & 5.5755 & -0.1206 & 0.0001 \\
$\mathrm{C}$ & -6.8340 & -0.3490 & -0.0002 \\
$\mathrm{C}$ & -8.1765 & 0.3947 & 0.0002 \\
$\mathrm{C}$ & 6.9246 & 0.6106 & 0.0001 \\
$\mathrm{C}$ & 8.1352 & -0.3325 & 0.0001 \\
$\mathrm{C}$ & -9.3963 & -0.5365 & -0.0001 \\
$\mathrm{C}$ & -10.7374 & 0.2096 & 0.0002 \\
$\mathrm{C}$ & 9.4829 & 0.4017 & 0.0001 \\
$\mathrm{C}$ & 10.6960 & -0.5382 & 0.0001 \\
$\mathrm{C}$ & -11.9586 & -0.7198 & -0.0001 \\
$\mathrm{C}$ & -13.2990 & 0.0276 & 0.0002 \\
$\mathrm{C}$ & 12.0423 & 0.1985 & 0.0000 \\
$\mathrm{C}$ & 13.2570 & -0.7393 & 0.0001 \\
$\mathrm{C}$ & -14.5208 & -0.9010 & -0.0002 \\
$\mathrm{C}$ & -15.8611 & -0.1538 & 0.0001 \\
$\mathrm{C}$ & 14.6025 & -0.0009 & -0.0001 \\
$\mathrm{C}$ & 15.8182 & -0.9371 & 0.0000 \\
$\mathrm{C}$ & -17.0830 & -1.0821 & -0.0002 \\
$\mathrm{C}$ & -18.4176 & -0.3280 & 0.0002 \\
$\mathrm{C}$ & 17.1633 & -0.1985 & -0.0002 \\
$\mathrm{C}$ & 18.3723 & -1.1408 & -0.0001 \\
$\mathrm{H}$ & -3.1067 & 1.4533 & -0.8745 \\
$\mathrm{H}$ & -3.1067 & 1.4526 & 0.8754 \\
$\mathrm{H}$ & -1.6386 & -0.6159 & -0.8740 \\
$\mathrm{H}$ & -1.6383 & -0.6162 & 0.8735 \\
$\mathrm{H}$ & 1.8464 & 1.7080 & 0.8834 \\
$\mathrm{H}$ & 1.8464 & 1.7076 & -0.8840 \\
$\mathrm{H}$ & 4.4266 & 1.4887 & -0.8790 \\
$\mathrm{H}$ & 2.9480 & -0.5603 & -0.8805 \\
$\mathrm{H}$ & 2.9478 & -0.5598 & 0.8810 \\
$\mathrm{H}$ & -4.2219 & -0.8175 & 0.8784 \\
$\mathrm{H}$ & -4.2219 & -0.8168 & -0.8793 \\
$\mathrm{H}$ & -5.6660 & 1.2483 & -0.8781 \\
\hline & -5.6660 & 1.2476 & 0.8790 \\
& & \\
$\mathrm{H}$
\end{tabular}




$\begin{array}{rrrr}\mathrm{H} & 5.5185 & -0.7818 & -0.8788 \\ \mathrm{H} & 5.5185 & -0.7817 & 0.8791 \\ \mathrm{H} & -6.7849 & -1.0118 & 0.8784 \\ \mathrm{H} & -6.7850 & -1.0110 & -0.8793 \\ \mathrm{H} & -8.2247 & 1.0577 & -0.8782 \\ \mathrm{H} & -8.2246 & 1.0570 & 0.8791 \\ \mathrm{H} & 6.9792 & 1.2726 & 0.8788 \\ \mathrm{H} & 6.9792 & 1.2725 & -0.8787 \\ \mathrm{H} & 8.0812 & -0.9945 & -0.8786 \\ \mathrm{H} & 8.0812 & -0.9944 & 0.8790 \\ \mathrm{H} & -9.3485 & -1.1994 & 0.8784 \\ \mathrm{H} & -9.3486 & -1.1987 & -0.8792 \\ \mathrm{H} & -10.7847 & 0.8726 & -0.8782 \\ \mathrm{H} & -10.7846 & 0.8720 & 0.8791 \\ \mathrm{H} & 9.5359 & 1.0640 & 0.8787 \\ \mathrm{H} & 9.5359 & 1.0638 & -0.8787 \\ \mathrm{H} & 10.6435 & -1.2005 & -0.8785 \\ \mathrm{H} & 10.6436 & -1.2003 & 0.8790 \\ \mathrm{H} & -11.9116 & -1.3828 & 0.8784 \\ \mathrm{H} & -11.9117 & -1.3822 & -0.8792 \\ \mathrm{H} & -13.3458 & 0.6907 & -0.8783 \\ \mathrm{H} & -13.3458 & 0.6900 & 0.8791 \\ \mathrm{H} & 12.0942 & 0.8610 & 0.8786 \\ \mathrm{H} & 12.0941 & 0.8607 & -0.8788 \\ \mathrm{H} & 13.2056 & -1.4018 & -0.8785 \\ \mathrm{H} & 13.2056 & -1.4014 & 0.8790 \\ \mathrm{H} & -14.4741 & -1.5641 & 0.8783 \\ \mathrm{H} & -14.4742 & -1.5634 & -0.8792 \\ \mathrm{H} & -15.9089 & 0.5094 & -0.8784 \\ \mathrm{H} & -15.9089 & 0.5087 & 0.8792 \\ \mathrm{H} & 14.6536 & 0.6617 & 0.8785 \\ \mathrm{H} & 14.6535 & 0.6613 & -0.8790 \\ \mathrm{H} & 15.7683 & -1.6000 & -0.8786 \\ \mathrm{H} & 15.7684 & -1.5996 & 0.8790 \\ \mathrm{H} & -17.0357 & -1.7441 & 0.8780 \\ \mathrm{H} & -17.0358 & -1.7433 & -0.8790 \\ \mathrm{H} & -19.2701 & -1.0194 & -0.0001 \\ \mathrm{H} & -18.5111 & 0.3158 & -0.8850 \\ \mathrm{H} & -18.5110 & 0.3151 & 0.8859 \\ \mathrm{H} & 17.2137 & 0.4631 & 0.8782 \\ \mathrm{H} & 17.2136 & 0.4628 & -0.8788 \\ \mathrm{H} & 19.3174 & -0.5828 & -0.0002 \\ & 18.3696 & -1.7912 & -0.8853 \\ & & -1.7909 & 0.8854\end{array}$

\section{6chain0}

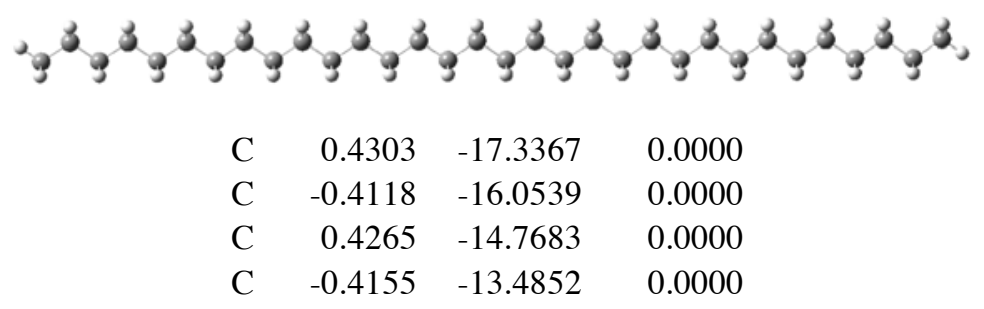




\begin{tabular}{|c|c|c|c|}
\hline & 4 & 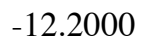 & 0 \\
\hline & -0.4180 & -10.9165 & 0.0000 \\
\hline & 0.4214 & -9.6317 & 0.0000 \\
\hline & -0.4195 & -8.3479 & 0.0000 \\
\hline & 0.4204 & -7.0633 & .0006 \\
\hline & -0.4202 & -5.7792 & 0.0000 \\
\hline & 0.4199 & -4.4949 & 0.0000 \\
\hline & -0.4204 & -3.2106 & 0.0000 \\
\hline & 0.4200 & -1.9264 & 0.0000 \\
\hline & -0.4202 & -0.6421 & 0.0000 \\
\hline & 0.4202 & 0.6421 & 0.000 \\
\hline & -0.4200 & 1.9264 & .0000 \\
\hline & -0.4070 & -18.6228 & 0.0000 \\
\hline & 0.4426 & -19.8987 & 0.0000 \\
\hline & 0.4204 & 3.2106 & 0.000 \\
\hline & -0.4199 & 4.4 & 0.0006 \\
\hline & 0.4202 & & 0.0000 \\
\hline & -0.4204 & 7.0633 & .0000 \\
\hline & 0.4195 & .3479 & .0000 \\
\hline & -0.4214 & 9.6317 & 0.0000 \\
\hline & 0.4180 & 10.9165 & 0.000 \\
\hline 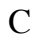 & -0.4234 & 12.2 & .0000 \\
\hline & 0.4155 & & .0000 \\
\hline & -0.4265 & 83 & .0000 \\
\hline & 0.4118 & 16.0539 & 0.0000 \\
\hline C & -0.4303 & 17.3367 & 0.000 \\
\hline C & 0.4070 & 18.6 & 0.0000 \\
\hline 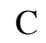 & -0.4426 & 19. & 0.0000 \\
\hline & 1.0949 & -17 & 0.8788 \\
\hline & 1.0949 & 173 & -0.8788 \\
\hline & -1.0761 & -16.0550 & 0.8788 \\
\hline & -1.0761 & -16.0550 & -0.8788 \\
\hline & 1.0908 & -14 & -0.8787 \\
\hline & 1.0908 & -14 & 0.8787 \\
\hline & -1.0798 & -13.4860 & 0.8787 \\
\hline & -1.0798 & -13.4860 & -0.8787 \\
\hline & 1.0878 & -12.1 & -0.8787 \\
\hline & 1.0878 & -12. & 0.8787 \\
\hline & -1.0823 & -10 & 0.8787 \\
\hline & -1.0823 & -10.9170 & -0.8787 \\
\hline & 1.0858 & -9.6313 & -0.8787 \\
\hline & 1.0858 & -9.6313 & 0.8787 \\
\hline & -1.0838 & -8.3481 & 0.8787 \\
\hline & -1.0838 & -8.3 & -0.8787 \\
\hline & 1.0847 & -7.0631 & -0.8787 \\
\hline & 1.0847 & -7.0631 & 0.8787 \\
\hline & -1.0845 & -5.7793 & .8787 \\
\hline & -1.0845 & -5.7793 & -0.8787 \\
\hline $\mathrm{H}$ & 1.0843 & -4.4948 & -0.8787 \\
\hline & 1.0843 & -4.4948 & 0.8787 \\
\hline $\mathrm{H}$ & -1.0847 & -3.2106 & 0.8787 \\
\hline 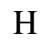 & -1.0847 & -3.2106 & -0.8787 \\
\hline 1 & 1.0843 & -1.9265 & -0.8787 \\
\hline $\mathrm{H}$ & 1.0843 & -1.9265 & 0.8787 \\
\hline & -1.0845 & -0.6420 & 0.878 \\
\hline
\end{tabular}




$\begin{array}{lrrr}\mathrm{H} & -1.0845 & -0.6420 & -0.8787 \\ \mathrm{H} & 1.0845 & 0.6420 & -0.8787 \\ \mathrm{H} & 1.0845 & 0.6420 & 0.8787 \\ \mathrm{H} & -1.0843 & 1.9265 & 0.8787 \\ \mathrm{H} & -1.0843 & 1.9265 & -0.8787 \\ \mathrm{H} & -1.0702 & -18.6235 & -0.8785 \\ \mathrm{H} & -1.0702 & -18.6235 & 0.8785 \\ \mathrm{H} & -0.1844 & -20.7996 & 0.0000 \\ \mathrm{H} & 1.0912 & -19.9445 & 0.8854 \\ \mathrm{H} & 1.0912 & -19.9445 & -0.8854 \\ \mathrm{H} & 1.0847 & 3.2106 & -0.8787 \\ \mathrm{H} & 1.0847 & 3.2106 & 0.8787 \\ \mathrm{H} & -1.0843 & 4.4948 & 0.8787 \\ \mathrm{H} & -1.0843 & 4.4948 & -0.8787 \\ \mathrm{H} & 1.0845 & 5.7793 & -0.8787 \\ \mathrm{H} & 1.0845 & 5.7793 & 0.8787 \\ \mathrm{H} & -1.0847 & 7.0631 & 0.8787 \\ \mathrm{H} & -1.0847 & 7.0631 & -0.8787 \\ \mathrm{H} & 1.0838 & 8.3481 & -0.8787 \\ \mathrm{H} & 1.0838 & 8.3481 & 0.8787 \\ \mathrm{H} & -1.0858 & 9.6313 & 0.8787 \\ \mathrm{H} & -1.0858 & 9.6313 & -0.8787 \\ \mathrm{H} & 1.0823 & 10.9170 & -0.8787 \\ \mathrm{H} & 1.0823 & 10.9170 & 0.8787 \\ \mathrm{H} & -1.0878 & 12.1994 & 0.8787 \\ \mathrm{H} & -1.0878 & 12.1994 & -0.8787 \\ \mathrm{H} & 1.0798 & 13.4860 & -0.8787 \\ \mathrm{H} & 1.0798 & 13.4860 & 0.8787 \\ \mathrm{H} & -1.0908 & 14.7674 & 0.8787 \\ \mathrm{H} & -1.0908 & 14.7674 & -0.8787 \\ \mathrm{H} & 1.0761 & 16.0550 & -0.8788 \\ \mathrm{H} & 1.0761 & 16.0550 & 0.8788 \\ \mathrm{H} & -1.0949 & 17.3365 & 0.8788 \\ \mathrm{H} & -1.0949 & 17.3365 & -0.8788 \\ \mathrm{H} & 1.0702 & 18.6235 & -0.8785 \\ \mathrm{H} & 1.0702 & 18.6235 & 0.8785 \\ \mathrm{H} & 0.1844 & 20.7996 & 0.0000 \\ \mathrm{H} & -1.0912 & 19.9445 & 0.8854 \\ \mathrm{H} & -1.0912 & 19.9445 & -0.8854\end{array}$

17chain0

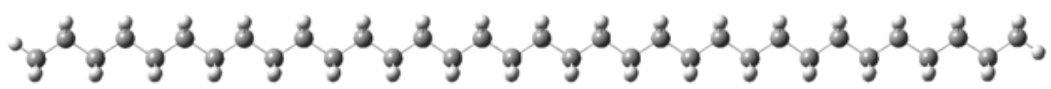

$\begin{array}{lrrr}\text { C } & 21.1839 & -0.3904 & 0.0000 \\ \text { C } & 19.9060 & 0.4564 & 0.0000 \\ \text { C } & 18.6218 & -0.3838 & 0.0000 \\ \text { C } & 17.3371 & 0.4555 & 0.0000 \\ \text { C } & 16.0535 & -0.3856 & 0.0000 \\ \text { C } & 14.7684 & 0.4535 & 0.0000 \\ \text { C } & 13.4852 & -0.3884 & -0.0001 \\ \text { C } & 12.1998 & 0.4501 & -0.0001 \\ \text { C } & 10.9169 & -0.3924 & -0.0001 \\ \text { C } & 9.6311 & 0.4455 & 0.0000 \\ & & \end{array}$




\begin{tabular}{|c|c|c|c|}
\hline & 8.3486 & -0 & 0.0000 \\
\hline & 7.0624 & 0.4400 & 0.0000 \\
\hline & 5.7802 & -0.4034 & 0.0000 \\
\hline & 4.4938 & 0.4337 & 0.0000 \\
\hline & 3.2118 & -0.4100 & 0.000 \\
\hline & 1.9252 & 0.4268 & 0.000 \\
\hline & 0.6433 & -0.4170 & 0.000 \\
\hline & -0.6433 & 0.4196 & 0.000 \\
\hline & -1.9252 & -0.4242 & 0.000 \\
\hline & -3.2118 & 0.4124 & 0.000 \\
\hline & -4.4937 & -0.4314 & 0.000 \\
\hline C & -5.7803 & 0.4054 & 0.000 \\
\hline & -7.0623 & -0.4382 & 0.000 \\
\hline & -8.3487 & 0.3988 & 0.000 \\
\hline & -9.6309 & -0.4445 & 0.000 \\
\hline & -10.9170 & .0929 & 0.000 \\
\hline $\mathrm{C}$ & -12.1 & -0.4500 & 0.000 \\
\hline & -13.4854 & 0.3879 & 0.000 \\
\hline & -14.7683 & -0.4545 & 0.0000 \\
\hline & -16.0537 & 0.3840 & 0.0000 \\
\hline ( & -17.3369 & -0.4578 & -0.000 \\
\hline$C$ & -18.6220 & & -0.0001 \\
\hline $\mathrm{C}$ & -19.9 & -0 . & 0.000 \\
\hline & -21.1841 & 61 & -0.000 \\
\hline & 22.0834 & 0.2385 & 0.0000 \\
\hline & 21.2311 & -1.0389 & -0.885 \\
\hline & 21.2310 & -1. & 0.885 \\
\hline $\mathrm{H}$ & 19.9054 & 97 & -0.878 \\
\hline $\mathrm{H}$ & 19.9054 & 96 & 0.878 \\
\hline & 18.6231 & 83 & 0.878 \\
\hline & 18.6231 & -1.0483 & -0.8788 \\
\hline F & 17.3368 & 1.1199 & -0.878 \\
\hline $\mathrm{H}$ & 17.3 & 99 & 0.878 \\
\hline $\mathrm{H}$ & 16.0 & 00 & $0.878^{\prime}$ \\
\hline & 16.0540 & -1.0499 & -0.878 \\
\hline & 14.7677 & 1.1178 & -0.878 \\
\hline & 14.7677 & 1178 & $0.878^{\prime}$ \\
\hline $\mathrm{H}$ & 13.4861 & -1. & $0.878^{\prime}$ \\
\hline $\mathrm{H}$ & 13.4 & -1 . & -0.8788 \\
\hline $\mathrm{H}$ & 12.1987 & 44 & -0.878 \\
\hline & 12.1987 & & $0.878^{7}$ \\
\hline & 10.9181 & -1.0567 & $0.878^{7}$ \\
\hline & 10.9181 & -1.0567 & -0.878 \\
\hline $\mathrm{H}$ & 9.62 & 98 & -0.878 \\
\hline $\mathrm{H}$ & 9.6298 & 98 & $0.878^{\prime}$ \\
\hline 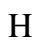 & 8.3500 & -1.0618 & $0.878^{\prime}$ \\
\hline & 8.3500 & -1.0618 & -0.878 \\
\hline F & 7.0609 & 1.1043 & -0.878 \\
\hline $\mathrm{H}$ & 7.0609 & 43 & $0.878^{\prime}$ \\
\hline 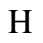 & 5.7818 & -1.0677 & $0.878^{\prime}$ \\
\hline $\mathrm{H}$ & 5.7818 & -1.0677 & $-0.878^{7}$ \\
\hline $\mathrm{H}$ & 4.4921 & 1.0980 & $-0.878^{\prime}$ \\
\hline & 4.4921 & 1.0980 & 0.878 \\
\hline F & 3.2135 & -1.0743 & 0.878 \\
\hline $\mathrm{H}$ & 3.2135 & -1.0743 & -0.878 \\
\hline
\end{tabular}




$\begin{array}{lrrr}\mathrm{H} & 1.9234 & 1.0911 & -0.8787 \\ \mathrm{H} & 1.9234 & 1.0912 & 0.8788 \\ \mathrm{H} & 0.6452 & -1.0813 & 0.8788 \\ \mathrm{H} & 0.6452 & -1.0814 & -0.8787 \\ \mathrm{H} & -0.6452 & 1.0840 & -0.8787 \\ \mathrm{H} & -0.6452 & 1.0840 & 0.8788 \\ \mathrm{H} & -1.9233 & -1.0886 & 0.8788 \\ \mathrm{H} & -1.9233 & -1.0886 & -0.8787 \\ \mathrm{H} & -3.2136 & 1.0768 & -0.8787 \\ \mathrm{H} & -3.2136 & 1.0768 & 0.8788 \\ \mathrm{H} & -4.4919 & -1.0957 & 0.8788 \\ \mathrm{H} & -4.4919 & -1.0957 & -0.8786 \\ \mathrm{H} & -5.7820 & 1.0697 & -0.8786 \\ \mathrm{H} & -5.7820 & 1.0697 & 0.8788 \\ \mathrm{H} & -7.0606 & -1.1026 & 0.8788 \\ \mathrm{H} & -7.0606 & -1.1026 & -0.8787 \\ \mathrm{H} & -8.3503 & 1.0632 & -0.8786 \\ \mathrm{H} & -8.3503 & 1.0631 & 0.8788 \\ \mathrm{H} & -9.6294 & -1.1088 & 0.8788 \\ \mathrm{H} & -9.6294 & -1.1088 & -0.8787 \\ \mathrm{H} & -10.9185 & 1.0573 & -0.8787 \\ \mathrm{H} & -10.9185 & 1.0572 & 0.8788 \\ \mathrm{H} & -12.1983 & -1.1143 & 0.8788 \\ \mathrm{H} & -12.1983 & -1.1143 & -0.8787 \\ \mathrm{H} & -13.4865 & 1.0523 & -0.8787 \\ \mathrm{H} & -13.4866 & 1.0522 & 0.8788 \\ \mathrm{H} & -14.7673 & -1.1188 & 0.8787 \\ \mathrm{H} & -14.7672 & -1.1188 & -0.8788 \\ \mathrm{H} & -16.0545 & 1.0483 & -0.8787 \\ \mathrm{H} & -16.0546 & 1.0483 & 0.8787 \\ \mathrm{H} & -17.3362 & -1.1221 & 0.8787 \\ \mathrm{H} & -17.3362 & -1.1221 & -0.8789 \\ \mathrm{H} & -18.6236 & 1.0454 & -0.8788 \\ \mathrm{H} & -18.6236 & 1.0454 & 0.8787 \\ \mathrm{H} & -19.9048 & -1.1232 & 0.8784 \\ \mathrm{H} & -19.9048 & -1.1232 & -0.8786 \\ \mathrm{H} & -22.0833 & -0.2432 & -0.0001 \\ \mathrm{H} & -21.2316 & 1.0346 & -0.8855 \\ \mathrm{H} & -21.2316 & 1.0346 & 0.8853 \\ & & & \end{array}$

17chain 1

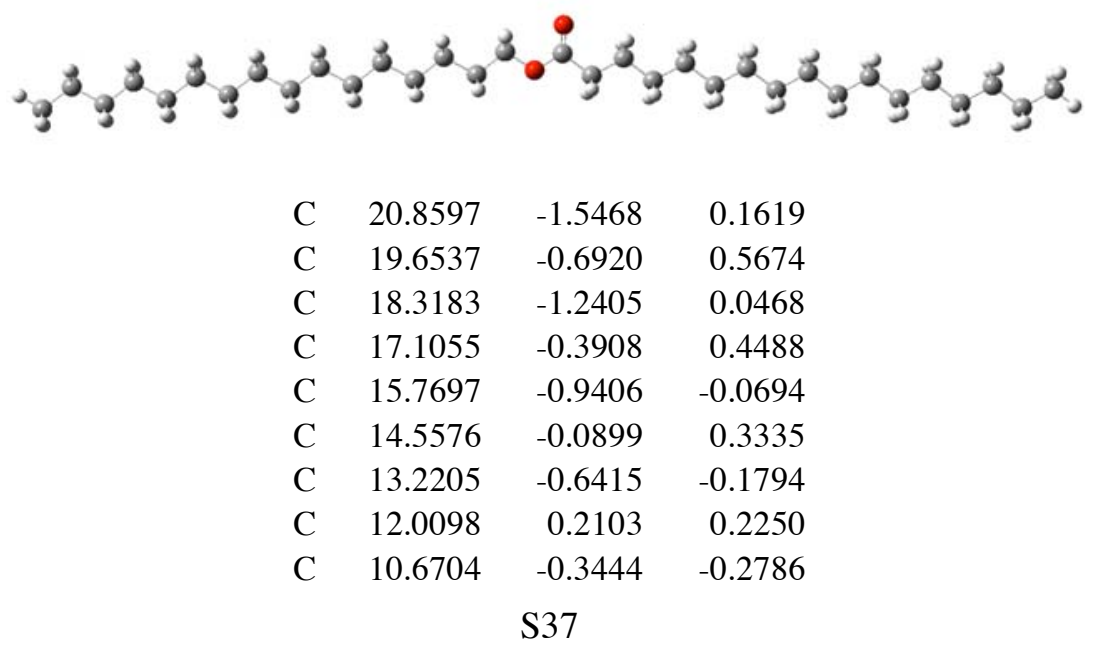




\begin{tabular}{|c|c|c|c|}
\hline & 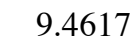 & & \\
\hline & 8.1191 & -0.0512 & -0.3599 \\
\hline & 6.9131 & 0.8039 & 0.0521 \\
\hline & 5.5668 & .2338 & -0.4139 \\
\hline & 4.3647 & .0910 & 0.005 \\
\hline & 3.0172 & 0.4995 & -0.4313 \\
\hline & 1.8428 & 1.3639 & 0.0032 \\
\hline & 0.6271 & 0.7026 & -0.4137 \\
\hline & -0.5284 & 1.3051 & -0.0507 \\
\hline & -1.7281 & 0.5089 & -0.5322 \\
\hline & -3.0620 & .0806 & -0.0460 \\
\hline & -4.2696 & & $-0.513 \varepsilon$ \\
\hline & -5.6093 & 0.8172 & -0.0156 \\
\hline & -6.8241 & -0.0094 & -0.4578 \\
\hline & -8.1606 & 0.5469 & 0.0518 \\
\hline & -9.3775 & 51 & $-0.374 \mathrm{C}$ \\
\hline 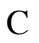 & 0.7 & & 0.142 \\
\hline 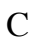 & -11.9297 & -0 . & -0.2742 \\
\hline & -13.2617 & & 0.2483 \\
\hline 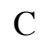 & -14.4810 & -0.8459 & -0.1624 \\
\hline C & -15.8117 & -0.2905 & 0.3629 \\
\hline 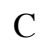 & -17.03 & 75 & -0.0447 \\
\hline E & -18.36 & & 0.4815 \\
\hline r & -19.5825 & & .0754 \\
\hline 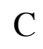 & -20.9064 & -0.8457 & 6046 \\
\hline & -0.5734 & 94 & .5744 \\
\hline & $21.7 \mathrm{c}$ & & 0.5499 \\
\hline & 20.9 & & -0.9308 \\
\hline & 20.7681 & & 0.5461 \\
\hline & 19.7922 & & 1985 \\
\hline & 19.6110 & -0.6153 & 6647 \\
\hline & 18.1807 & -2.2696 & 0.4152 \\
\hline & 18.3614 & -1 & -1.0514 \\
\hline & 17.2 & & 0.0791 \\
\hline & 17.0647 & -0.3123 & .5469 \\
\hline & 15.6315 & & .3009 \\
\hline & 15.8101 & & 1.1674 \\
\hline & 14.6944 & 6 & -0.0394 \\
\hline & 14.5 & -0 . & 1.4314 \\
\hline & 13.0836 & & 1943 \\
\hline & 13.2575 & -0.7236 & -1.2773 \\
\hline & 12.1442 & 2362 & -0.1531 \\
\hline & 11.9769 & 2968 & 1.3228 \\
\hline & 10.5 & -1.7 & 1008 \\
\hline I & 10.7015 & -0.4316 & -1.3763 \\
\hline & 9.5919 & 1.5322 & -0.2578 \\
\hline & 9.4371 & & 2261 \\
\hline & 7.9891 & -1.0739 & .0284 \\
\hline $\mathrm{H}$ & 8.1408 & -0.1465 & -1.4571 \\
\hline 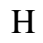 & 7.0358 & 1.8230 & -0.3475 \\
\hline 1 & 6.9012 & 9103 & 1.1484 \\
\hline & 5.4439 & -0.7842 & -0.0119 \\
\hline & 5.5737 & 0.1265 & -1.5099 \\
\hline & 4.4763 & 2.1034 & -0.4136 \\
\hline & 4.3716 & 1.2143 & 1.099 \\
\hline
\end{tabular}




$\begin{array}{lrrr}\mathrm{H} & 2.8935 & -0.5063 & -0.0050 \\ \mathrm{H} & 2.9917 & 0.3805 & -1.5238 \\ \mathrm{H} & 1.8781 & 2.3630 & -0.4498 \\ \mathrm{H} & 1.8164 & 1.5002 & 1.0916 \\ \mathrm{H} & -1.5956 & -0.5323 & -0.2051 \\ \mathrm{H} & -1.6876 & 0.4739 & -1.6311 \\ \mathrm{H} & -3.1565 & 2.1190 & -0.3913 \\ \mathrm{H} & -3.0504 & 1.1341 & 1.0514 \\ \mathrm{H} & -4.1598 & -0.7824 & -0.1698 \\ \mathrm{H} & -4.2803 & 0.2117 & -1.6145 \\ \mathrm{H} & -5.7248 & 1.8540 & -0.3688 \\ \mathrm{H} & -5.5902 & 0.8751 & 1.0841 \\ \mathrm{H} & -6.7048 & -1.0469 & -0.1070 \\ \mathrm{H} & -6.8477 & -0.0647 & -1.5578 \\ \mathrm{H} & -8.2838 & 1.5817 & -0.3050 \\ \mathrm{H} & -8.1315 & 0.6098 & 1.1511 \\ \mathrm{H} & -9.2525 & -1.3204 & -0.0198 \\ \mathrm{H} & -9.4102 & -0.3463 & -1.4743 \\ \mathrm{H} & -10.8388 & 1.3040 & -0.2162 \\ \mathrm{H} & -10.6751 & 0.3362 & 1.2416 \\ \mathrm{H} & -11.8013 & -1.5987 & 0.0835 \\ \mathrm{H} & -11.9683 & -0.6295 & -1.3732 \\ \mathrm{H} & -13.3914 & 1.0236 & -0.1115 \\ \mathrm{H} & -13.2209 & 0.0581 & 1.3471 \\ \mathrm{H} & -14.3509 & -1.8791 & 0.1969 \\ \mathrm{H} & -14.5231 & -0.9130 & -1.2612 \\ \mathrm{H} & -15.9425 & 0.7423 & 0.0025 \\ \mathrm{H} & -15.7685 & -0.2220 & 1.4615 \\ \mathrm{H} & -16.9010 & -2.1603 & 0.3157 \\ \mathrm{H} & -17.0755 & -1.1959 & -1.1433 \\ \mathrm{H} & -18.4940 & 0.4607 & 0.1209 \\ \mathrm{H} & -18.3189 & -0.5030 & 1.5803 \\ \mathrm{H} & -19.4518 & -2.4401 & 0.4370 \\ \mathrm{H} & -19.6261 & -1.4774 & -1.0224 \\ \mathrm{H} & -21.7586 & -1.4656 & 0.2976 \\ \mathrm{H} & -21.0834 & 0.1720 & 0.2308 \\ \mathrm{H} & -20.9080 & -0.7978 & 1.7021\end{array}$

17chain2

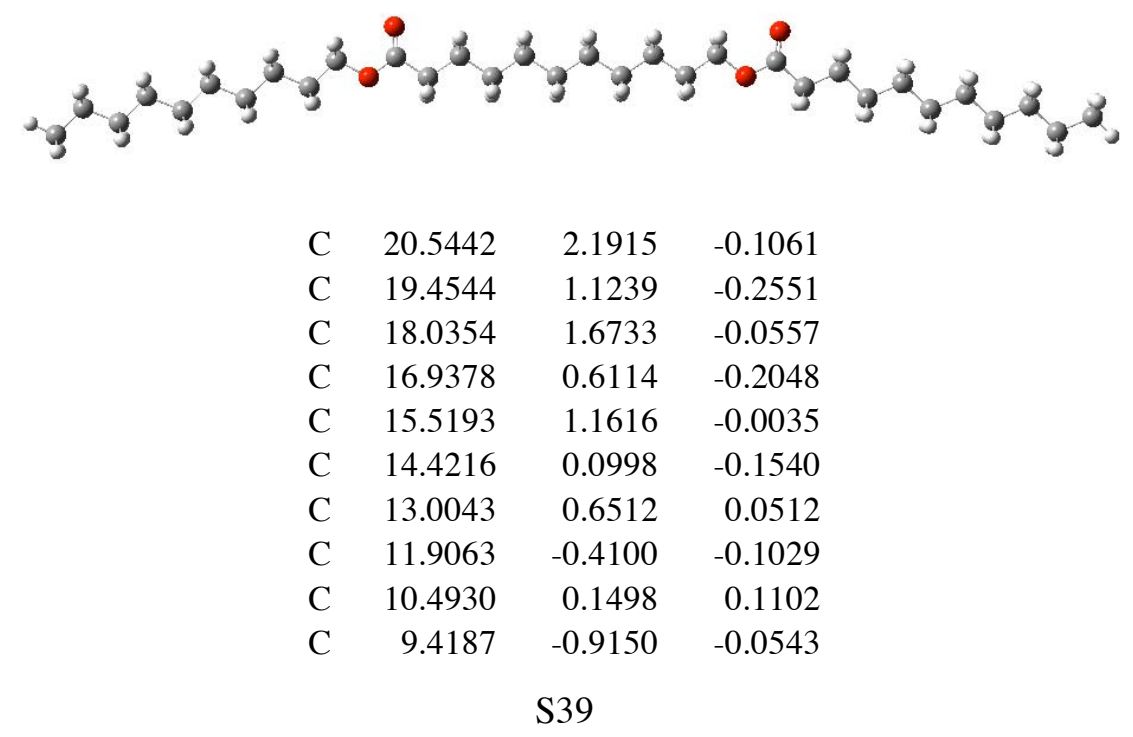




\begin{tabular}{|c|c|c|c|}
\hline O & 8.1365 & -0.2857 & 0.1687 \\
\hline $\mathrm{C}$ & 7.0527 & -1.0837 & 0.0348 \\
\hline $\mathrm{C}$ & 5.7754 & -0.3074 & 0.3008 \\
\hline C & 4.5087 & -1.1314 & 0.0588 \\
\hline $\mathrm{C}$ & 3.2234 & -0.3405 & 0.3332 \\
\hline C & 1.9469 & -1.1504 & 0.0701 \\
\hline$C$ & 0.6557 & -0.3637 & 0.3320 \\
\hline $\mathrm{C}$ & -0.6194 & -1.1717 & 0.0557 \\
\hline $\mathrm{C}$ & -1.9117 & -0.3801 & 0.2961 \\
\hline $\mathrm{C}$ & -3.1848 & -1.1898 & 0.0147 \\
\hline C & -4.4729 & -0.3820 & 0.2242 \\
\hline $\mathrm{C}$ & -5.7221 & -1.2053 & -0.0532 \\
\hline $\mathrm{O}$ & -6.8679 & -0.3433 & 0.1288 \\
\hline $\mathrm{C}$ & -8.0772 & -0.9120 & -0.0818 \\
\hline $\mathrm{C}$ & -9.1906 & 0.1000 & 0.1200 \\
\hline $\mathrm{C}$ & -10.5877 & -0.4899 & -0.0865 \\
\hline $\mathrm{C}$ & -11.7019 & 0.5491 & 0.0926 \\
\hline $\mathrm{C}$ & -13.1095 & -0.0290 & -0.1069 \\
\hline $\mathrm{C}$ & -14.2269 & 1.0100 & 0.0571 \\
\hline $\mathrm{C}$ & -15.6358 & 0.4337 & -0.1379 \\
\hline $\mathrm{C}$ & -16.7520 & 1.4751 & 0.0191 \\
\hline $\mathrm{C}$ & -18.1617 & 0.8997 & -0.1721 \\
\hline $\mathrm{C}$ & -19.2777 & 1.9418 & -0.0190 \\
\hline $\mathrm{C}$ & -20.6825 & 1.3578 & -0.2073 \\
\hline $\mathrm{O}$ & 7.1186 & -2.2559 & -0.2553 \\
\hline $\mathrm{O}$ & -8.2243 & -2.0716 & -0.3913 \\
\hline $\mathrm{H}$ & 21.5454 & 1.7674 & -0.2549 \\
\hline $\mathrm{H}$ & 20.5208 & 2.6470 & 0.8933 \\
\hline $\mathrm{H}$ & 20.4126 & 2.9992 & -0.8389 \\
\hline $\mathrm{H}$ & 19.6341 & 0.3131 & 0.4673 \\
\hline $\mathrm{H}$ & 19.5276 & 0.6617 & -1.2515 \\
\hline $\mathrm{H}$ & 17.8567 & 2.4862 & -0.7775 \\
\hline $\mathrm{H}$ & 17.9623 & 2.1357 & 0.9416 \\
\hline $\mathrm{H}$ & 17.1181 & -0.2018 & 0.5160 \\
\hline $\mathrm{H}$ & 17.0110 & 0.1505 & -1.2027 \\
\hline $\mathrm{H}$ & 15.3393 & 1.9754 & -0.7237 \\
\hline $\mathrm{H}$ & 15.4459 & 1.6217 & 0.9947 \\
\hline $\mathrm{H}$ & 14.6023 & -0.7154 & 0.5643 \\
\hline $\mathrm{H}$ & 14.4928 & -0.3582 & -1.1533 \\
\hline $\mathrm{H}$ & 12.8232 & 1.4673 & -0.6659 \\
\hline $\mathrm{H}$ & 12.9313 & 1.1068 & 1.0512 \\
\hline $\mathrm{H}$ & 12.0886 & -1.2285 & 0.6109 \\
\hline $\mathrm{H}$ & 11.9755 & -0.8616 & -1.1049 \\
\hline $\mathrm{H}$ & 10.2995 & 0.9637 & -0.6031 \\
\hline $\mathrm{H}$ & 10.4096 & 0.5891 & 1.1144 \\
\hline $\mathrm{H}$ & 9.5412 & -1.7361 & 0.6635 \\
\hline $\mathrm{H}$ & 9.4284 & -1.3554 & -1.0593 \\
\hline $\mathrm{H}$ & 5.7916 & 0.5960 & -0.3254 \\
\hline $\mathrm{H}$ & 5.8181 & 0.0589 & 1.3374 \\
\hline $\mathrm{H}$ & 4.5386 & -2.0313 & 0.6879 \\
\hline $\mathrm{H}$ & 4.5090 & -1.4953 & -0.9780 \\
\hline $\mathrm{H}$ & 3.2115 & 0.5682 & -0.2898 \\
\hline$\Pi$ & 3.2235 & 0.0113 & 1.3774 \\
\hline $\mathrm{H}$ & 1.9558 & -2.0570 & 0.6954 \\
\hline $\mathrm{H}$ & 1.9514 & -1.5046 & -0.9727 \\
\hline
\end{tabular}




$\begin{array}{rrrr}\mathrm{H} & 0.6516 & 0.5453 & -0.2904 \\ \mathrm{H} & 0.6465 & -0.0135 & 1.3763 \\ \mathrm{H} & -0.6221 & -2.0752 & 0.6856 \\ \mathrm{H} & -0.6029 & -1.5310 & -0.9854 \\ \mathrm{H} & -1.9086 & 0.5220 & -0.3358 \\ \mathrm{H} & -1.9323 & -0.0195 & 1.3365 \\ \mathrm{H} & -3.1990 & -2.0815 & 0.6608 \\ \mathrm{H} & -3.1538 & -1.5666 & -1.0196 \\ \mathrm{H} & -4.4748 & 0.4986 & -0.4339 \\ \mathrm{H} & -4.5167 & -0.0011 & 1.2546 \\ \mathrm{H} & -5.8091 & -2.0622 & 0.6267 \\ \mathrm{H} & -5.7337 & -1.5988 & -1.0777 \\ \mathrm{H} & -9.0079 & 0.9385 & -0.5680 \\ \mathrm{H} & -9.0817 & 0.5255 & 1.1282 \\ \mathrm{H} & -10.7346 & -1.3229 & 0.6145 \\ \mathrm{H} & -10.6463 & -0.9335 & -1.0897 \\ \mathrm{H} & -11.5464 & 1.3787 & -0.6156 \\ \mathrm{H} & -11.6312 & 0.9951 & 1.0975 \\ \mathrm{H} & -13.2685 & -0.8522 & 0.6072 \\ \mathrm{H} & -13.1774 & -0.4822 & -1.1083 \\ \mathrm{H} & -14.0677 & 1.8312 & -0.6598 \\ \mathrm{H} & -14.1559 & 1.4662 & 1.0573 \\ \mathrm{H} & -15.7969 & -0.3842 & 0.5821 \\ \mathrm{H} & -15.7056 & -0.0266 & -1.1362 \\ \mathrm{H} & -16.5917 & 2.2914 & -0.7030 \\ \mathrm{H} & -16.6804 & 1.9376 & 1.0164 \\ \mathrm{H} & -18.3241 & 0.0851 & 0.5517 \\ \mathrm{H} & -18.2339 & 0.4349 & -1.1683 \\ \mathrm{H} & -19.1179 & 2.7535 & -0.7452 \\ \mathrm{H} & -19.2047 & 2.4081 & 0.9755 \\ \mathrm{H} & -21.4564 & 2.1282 & -0.0971 \\ \mathrm{H} & -20.8885 & 0.5710 & 0.5311 \\ \mathrm{H} & -20.7971 & 0.9111 & -1.2044 \\ & & & \end{array}$

17chain3

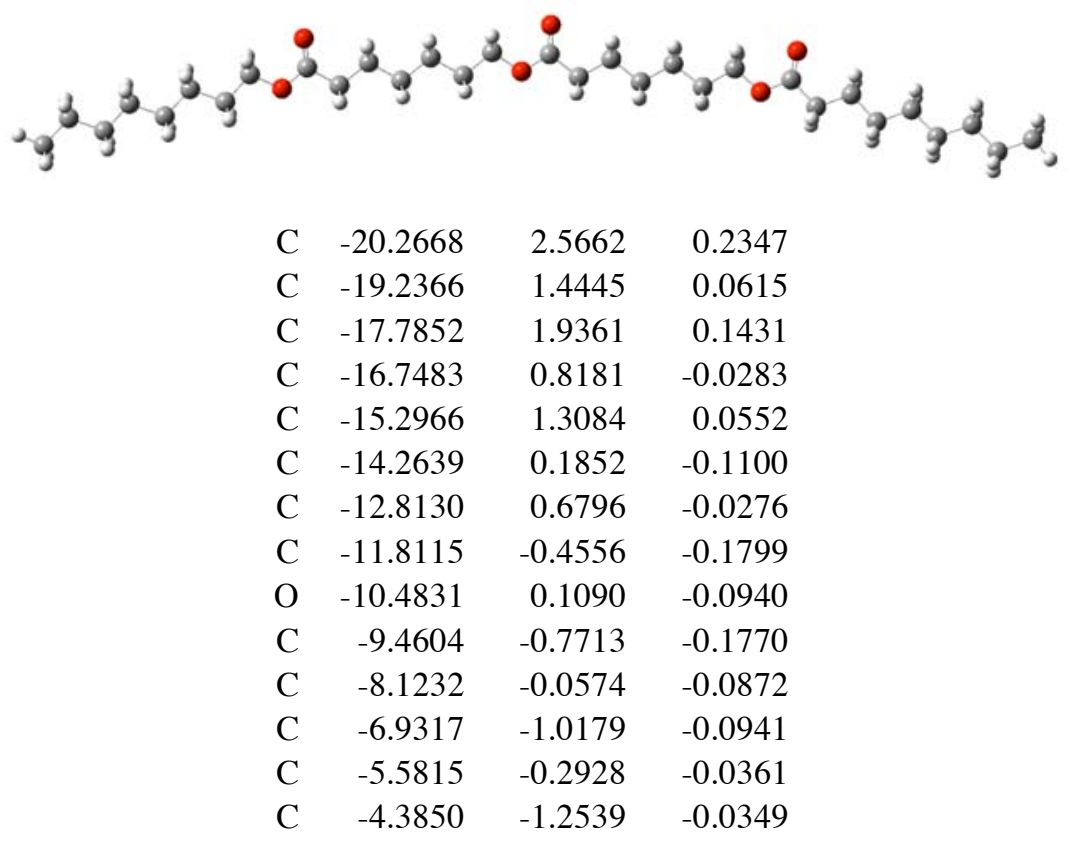




\begin{tabular}{|c|c|c|c|}
\hline & 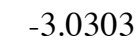 & & \\
\hline & -1.8633 & -1.5098 & 001 \\
\hline & -0.6403 & -0.7392 & 0.0382 \\
\hline & 0.5074 & -1.4543 & 33 \\
\hline & 1.7171 & 0.5366 & .065 \\
\hline & 3.0440 & -1.2984 & 1026 \\
\hline C & 4.2646 & -0.3699 & 85 \\
\hline & 5.5970 & -1.1309 & 0.120 \\
\hline$c$ & 6.8217 & -0.2068 & 0.080 \\
\hline 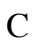 & 8.1290 & -0.9845 & 0.1207 \\
\hline & 9.2131 & -0.0316 & \\
\hline$C$ & 10.4614 & & \\
\hline $\mathrm{C}$ & 11.5071 & 0.5423 & -0.029 \\
\hline$C$ & 12.9404 & 0.0193 & 0.091 \\
\hline$c$ & 13.9928 & 1.1231 & $-0.072 ?$ \\
\hline$c$ & 15.4334 & 0.6079 & 0.047 \\
\hline$C$ & 16. & & 0.131 \\
\hline $\mathrm{C}$ & 17.9341 & & -0.012 \\
\hline & 18.9958 & 94 & 0.1980 \\
\hline & 20.4315 & 1.7556 & $-0.078 \varepsilon$ \\
\hline & 0.5411 & -2.6630 & 0.053 \\
\hline$c$ & 10.6 & & 0.176 \\
\hline 0 & -9.6 & & -0.309 \\
\hline $\mathrm{H}$ & -21.2931 & & 0.1740 \\
\hline & -20.1528 & 3.3349 & -0.5417 \\
\hline & -20.1543 & 0626 & 1.2082 \\
\hline $\mathrm{H}$ & -19.3965 & 53 & -0.906 \\
\hline $\mathrm{H}$ & -19 & & 29. \\
\hline$H_{-}$ & -17.6 & 64 & 1.111 \\
\hline & -17.6197 & & -0.625 \\
\hline & -16.9084 & 3185 & -0.996 \\
\hline & -16.9157 & 0462 & 0.739 \\
\hline $\mathrm{H}$ & -15 . & & 1.022 \\
\hline $\mathrm{H}$ & -15 . & 64 & -0.715 \\
\hline $\mathrm{H}$ & -14.4257 & 83 & -1.075 \\
\hline & -14.4346 & & 0.662 \\
\hline & -12.6402 & 14 & 0.935 \\
\hline $\mathrm{H}$ & -12.6255 & 299 & -0.808 \\
\hline $\mathrm{H}$ & -11. & 33 & -1.144 \\
\hline $\mathrm{H}$ & -11.9268 & -1 & 0.606 \\
\hline & -8.1308 & & 0.817 \\
\hline & -8.0665 & & -0.926 \\
\hline $\mathrm{H}$ & -6.9821 & 59 & -0.993 \\
\hline $\mathrm{H}$ & -7.0218 & 97 & 0.754 \\
\hline$U$ & -5.53 & 08 & 0.863 \\
\hline $\mathrm{H}$ & -5.4954 & & -0.892 \\
\hline $\mathrm{H}$ & -4.4334 & -1.8938 & -0.929 \\
\hline H & -4.4672 & -1.9336 & 0.827 \\
\hline $\mathrm{H}$ & -2.9690 & 01015 & 0.899 \\
\hline 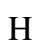 & -2.9318 & 38 & -0.861 \\
\hline $\mathrm{H}$ & -1.8606 & -2.1519 & -0.875 \\
\hline $\mathrm{H}$ & -1.8853 & -2.1684 & 0.891 \\
\hline $\mathrm{H}$ & 1.6 & 404 & 0.926 \\
\hline & 1.6550 & 0.1117 & -0.820 \\
\hline & 3.0858 & -1.9878 & -0.751 \\
\hline
\end{tabular}




$\begin{array}{rrrr}\mathrm{H} & 3.0694 & -1.9348 & 0.9977 \\ \mathrm{H} & 4.2170 & 0.3206 & 0.9420 \\ \mathrm{H} & 4.2325 & 0.2639 & -0.8151 \\ \mathrm{H} & 5.6370 & -1.8304 & -0.7283 \\ \mathrm{H} & 5.6344 & -1.7542 & 1.0278 \\ \mathrm{H} & 6.7954 & 0.4928 & 0.9283 \\ \mathrm{H} & 6.8001 & 0.4067 & -0.8313 \\ \mathrm{H} & 8.2143 & -1.6840 & -0.7208 \\ \mathrm{H} & 8.2302 & -1.5701 & 1.0431 \\ \mathrm{H} & 11.2890 & 1.2998 & 0.7372 \\ \mathrm{H} & 11.3529 & 1.0526 & -0.9916 \\ \mathrm{H} & 13.0999 & -0.7659 & -0.6593 \\ \mathrm{H} & 13.0638 & -0.4739 & 1.0660 \\ \mathrm{H} & 13.8236 & 1.9093 & 0.6807 \\ \mathrm{H} & 13.8616 & 1.6117 & -1.0511 \\ \mathrm{H} & 15.5981 & -0.1844 & -0.6996 \\ \mathrm{H} & 15.5669 & 0.1276 & 1.0294 \\ \mathrm{H} & 16.3284 & 2.4959 & 0.6149 \\ \mathrm{H} & 16.3593 & 2.1819 & -1.1144 \\ \mathrm{H} & 18.0988 & 0.3911 & -0.7560 \\ \mathrm{H} & 18.0708 & 0.7113 & 0.9718 \\ \mathrm{H} & 18.8327 & 3.0743 & 0.5459 \\ \mathrm{H} & 18.8590 & 2.7542 & -1.1816 \\ \mathrm{H} & 21.1655 & 2.5600 & -0.2157 \\ \mathrm{H} & 20.6377 & 0.9848 & -0.8338 \\ \mathrm{H} & 20.6103 & 1.3061 & 0.9074\end{array}$

17chain4

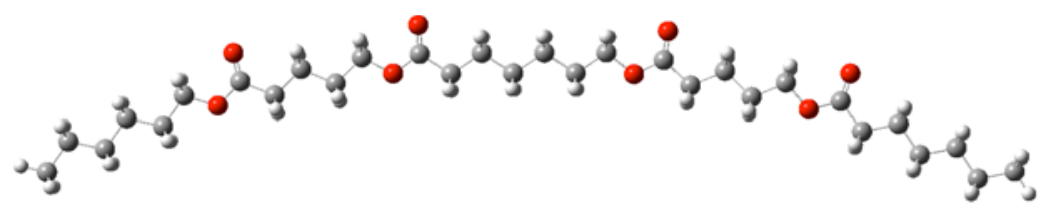

$\begin{array}{rrrr}\text { C } & 19.6759 & 3.5281 & -0.0515 \\ \text { C } & 18.8269 & 2.2520 & -0.0649 \\ \text { C } & 17.3188 & 2.5293 & -0.0057 \\ \text { C } & 16.4603 & 1.2574 & -0.0210 \\ \text { C } & 14.9541 & 1.5478 & 0.0377 \\ \text { C } & 14.1181 & 0.2768 & 0.0146 \\ \text { O } & 12.7261 & 0.6655 & 0.0712 \\ \text { C } & 11.8261 & -0.3412 & 0.0292 \\ \text { C } & 10.4092 & 0.2006 & 0.1019 \\ \text { C } & 9.3455 & -0.8917 & -0.0305 \\ \text { C } & 7.9208 & -0.3291 & 0.0535 \\ \text { C } & 6.8662 & -1.4180 & -0.0807 \\ \text { O } & 5.5710 & -0.7823 & -0.0002 \\ \text { C } & 4.4999 & -1.6036 & -0.0887 \\ \text { C } & 3.2069 & -0.8121 & -0.0062 \\ \text { C } & 1.9581 & -1.6968 & -0.0241 \\ \text { C } & 0.6587 & -0.8840 & 0.0323 \\ \text { C } & -0.6030 & -1.7577 & 0.0169 \\ \text { C } & -1.8988 & -0.9368 & 0.0699 \\ \text { C } & -3.1422 & -1.8135 & 0.0489 \\ \text { O } & -4.2937 & -0.9408 & 0.1068 \\ & & \text { S43 } & \end{array}$




\begin{tabular}{|c|c|c|c|}
\hline & 4 & 63 & \\
\hline & -6.6182 & -0.5204 & \\
\hline & -8.0119 & -1.1424 & 0345 \\
\hline & -9.1247 & -0.0907 & 0.1268 \\
\hline & -10.5112 & -0.7063 & 004 \\
\hline & -11.4715 & .3693 & 089 \\
\hline & -12.7732 & .0158 & 0.023 \\
\hline & -13.6699 & 1.2371 & 0.066 \\
\hline & -15.1601 & 0.9048 & -0.0403 \\
\hline & -16.0 & 2.1533 & 0.024 \\
\hline & -17. & & -0.081 \\
\hline & -18. & & 267 \\
\hline & -19.9341 & .7721 & 0.1314 \\
\hline & 12.1289 & 90 & 0.0524 \\
\hline & 4.5862 & -2.8022 & -0.220 \\
\hline & -5.6 & -2 & \\
\hline & -13.1 & & -0.178 \\
\hline & 20.7478 & & -0.0941 \\
\hline & 19.4977 & & 0.859 \\
\hline & 19.4397 & 4.1720 & -0.909 \\
\hline & 19.1 & 1.6124 & 0.784 \\
\hline & 19. & & -0.971 \\
\hline & 17.0 & & \\
\hline & 17.0 & & 0.901 \\
\hline & 16.7445 & 0.6162 & 0.828 \\
\hline & 16.68 & 0.6764 & -0.928 \\
\hline & 14. & & -0 . \\
\hline & 14. & & 48 \\
\hline & 14. & & 68 \\
\hline & 14.2858 & -0 . & $-0.897\}$ \\
\hline & 10.2972 & & -0.679 \\
\hline $\mathrm{H}$ & 10.3054 & 7409 & 1.054 \\
\hline $\mathrm{H}$ & 9 & 47 & 53 \\
\hline I & & & 83 \\
\hline & 7.7609 & & -0.736 \\
\hline & 7.7739 & & 1.010 \\
\hline & 6.9462 & -2.1678 & 0.716 \\
\hline $\mathrm{H}$ & 6.9444 & & 1. \\
\hline 1 & 90 & 94 & -0 \\
\hline $\mathrm{H}$ & & & 0.900 \\
\hline & & & 0.8214 \\
\hline $\mathrm{H}$ & 1.9724 & -2.3214 & -0.927 \\
\hline $\mathrm{H}$ & 0.6256 & -0 & -0.818 \\
\hline $\mathrm{H}$ & 7 & -0 & $0 c$ \\
\hline 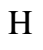 & -0.5729 & & 0.868 \\
\hline $\mathrm{H}$ & -0.5998 & & -0.888 \\
\hline & -1.9391 & & -0.781 \\
\hline $\mathrm{H}$ & -1.9148 & & 0.978 \\
\hline $\mathrm{H}$ & -3.1746 & & 0.903 \\
\hline 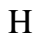 & -3.2018 & -2.4209 & -0.862 \\
\hline $\mathrm{H}$ & -6.4481 & & -0.637 \\
\hline $\mathrm{H}$ & & & 1.097 \\
\hline & & & 0.8222 \\
\hline & -8.0855 & -1.6887 & -0.915 \\
\hline & -9.0015 & 0.6624 & -0.664 \\
\hline
\end{tabular}




$\begin{array}{rrrr}\mathrm{H} & -9.0585 & 0.4493 & 1.0823 \\ \mathrm{H} & -10.7119 & -1.4295 & 0.8051 \\ \mathrm{H} & -10.6445 & -1.2301 & -0.9503 \\ \mathrm{H} & -13.3615 & 1.9366 & -0.7239 \\ \mathrm{H} & -13.4429 & 1.7522 & 1.0116 \\ \mathrm{H} & -15.4333 & 0.2068 & 0.7629 \\ \mathrm{H} & -15.3416 & 0.3627 & -0.9783 \\ \mathrm{H} & -15.7652 & 2.8465 & -0.7838 \\ \mathrm{H} & -15.8560 & 2.6938 & 0.9647 \\ \mathrm{H} & -17.8344 & 1.1532 & 0.7301 \\ \mathrm{H} & -17.7409 & 1.2964 & -1.0191 \\ \mathrm{H} & -18.1510 & 3.7749 & -0.8385 \\ \mathrm{H} & -18.2433 & 3.6330 & 0.9104 \\ \mathrm{H} & -20.5420 & 3.6848 & -0.0873 \\ \mathrm{H} & -20.2599 & 2.1153 & 0.6865 \\ \mathrm{H} & -20.1677 & 2.2620 & -1.0758\end{array}$

17chain5

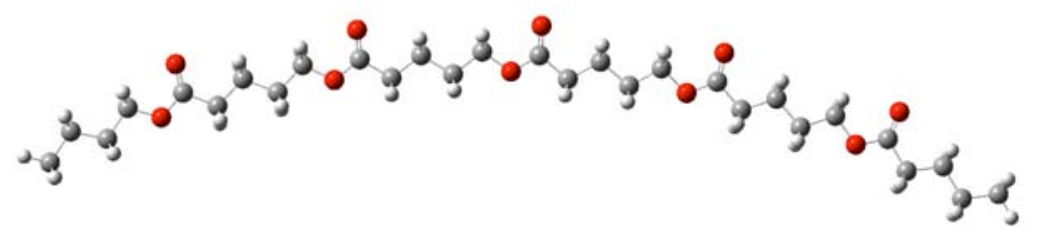

$\begin{array}{lrrr}\text { C } & 19.4830 & 3.5751 & -0.0090 \\ \text { C } & 18.6859 & 2.2661 & -0.0258 \\ \text { C } & 17.1682 & 2.4915 & 0.0211 \\ \text { C } & 16.3920 & 1.1833 & -0.0021 \\ \text { O } & 14.9821 & 1.5023 & 0.0458 \\ \text { C } & 14.1369 & 0.4492 & 0.0056 \\ \text { C } & 12.6919 & 0.9125 & 0.0692 \\ \text { C } & 11.6944 & -0.2422 & -0.0494 \\ \text { C } & 10.2373 & 0.2313 & 0.0284 \\ \text { C } & 9.2590 & -0.9281 & -0.0913 \\ \text { O } & 7.9211 & -0.3862 & -0.0118 \\ \text { C } & 6.9147 & -1.2856 & -0.0839 \\ \text { C } & 5.5651 & -0.5948 & 0.0025 \\ \text { C } & 4.3931 & -1.5784 & -0.0329 \\ \text { C } & 3.0331 & -0.8720 & 0.0339 \\ \text { C } & 1.8781 & -1.8622 & -0.0007 \\ \text { O } & 0.6477 & -1.1052 & 0.0560 \\ \text { C } & -0.4930 & -1.8300 & 0.0272 \\ \text { C } & -1.7103 & -0.9240 & 0.0849 \\ \text { C } & -3.0289 & -1.7006 & 0.0595 \\ \text { C } & -4.2527 & -0.7767 & 0.1045 \\ \text { C } & -5.5569 & -1.5603 & 0.0717 \\ \text { O } & -6.6427 & -0.6074 & 0.1194 \\ \text { C } & -7.8894 & -1.1288 & 0.0774 \\ \text { C } & -8.9360 & -0.0307 & 0.1444 \\ \text { C } & -10.3653 & -0.5632 & 0.0185 \\ \text { C } & -11.4139 & 0.5529 & 0.1079 \\ \text { C } & -12.8316 & 0.0153 & -0.0243 \\ \text { O } & -13.7348 & 1.1397 & 0.0563 \\ \text { C } & -15.0520 & 0.8510 & -0.0620 \\ & & \text { S45 } & \\ & & & \end{array}$




\begin{tabular}{|c|c|c|c|}
\hline & 4 & & \\
\hline & -17.3933 & 1.8470 & -0.0864 \\
\hline & -18.2315 & .1298 & -0.0201 \\
\hline & -19.7374 & 65 & .1310 \\
\hline & 14.5022 & -0.7011 & -0.0682 \\
\hline & 7.0879 & -2.4760 & -0.2043 \\
\hline & -0.5179 & -3.0368 & 0.037 \\
\hline & -8.1171 & -2.3136 & $0.000 s$ \\
\hline & -15.4767 & -0.2699 & -0.2189 \\
\hline & 20.5628 & 3.3840 & -0.042 \\
\hline & 19.2748 & 4.1561 & 0.899 \\
\hline & 19.2289 & 4.2065 & -0.8709 \\
\hline & 18.9895 & 1.6411 & 0.827 \\
\hline & 18.9428 & 1.6924 & -0.9290 \\
\hline & 16.8537 & 3.1091 & -0.832 \\
\hline & 16.8 & 3.0 & 0.928 \\
\hline & 16.6 & & \\
\hline & 16.5930 & 0.6 & -0.911 \\
\hline & 12.5382 & 87 & -0.7232 \\
\hline & 12.5555 & 1.4 & 1.013 \\
\hline & 11.8978 & -0.9 & 0.743 \\
\hline & 11.8 & -0 & -0.995 \\
\hline & 10.0 & & $-0.770^{\top}$ \\
\hline & & & \\
\hline & 9.3939 & -1.6628 & 0.7122 \\
\hline & 9.3708 & -1.4 & -1.043 \\
\hline & & 0 & -0.825 \\
\hline & & & 0.919 \\
\hline & 4 & -2.2 & 0.800 \\
\hline & 09 & 2 & -0.946 \\
\hline & 269 & 5 & -0.807 \\
\hline $\mathrm{H}$ & 2.9602 & -0.2 & 0.951 \\
\hline $\mathrm{H}$ & 2 & - & 48 \\
\hline & & -2 & -0.917 \\
\hline & -1.6468 & -0.2 & -0.756 \\
\hline & -1.6291 & -0.3 & 0.989 \\
\hline & -3.0507 & -2.3 & 0.906 \\
\hline $\mathrm{H}$ & -3 & -23 & $-0.842^{\prime}$ \\
\hline F & -4 & 03 & -0.746 \\
\hline $\mathrm{H}_{\mathrm{H}}$ & -4.2305 & -0.1 & 1.013 \\
\hline & & -2.2444 & 0.925 \\
\hline & -5.65 & -2.1618 & -0.840 \\
\hline $\mathrm{H}$ & -8. & 0.7031 & -0.642 \\
\hline $\mathrm{H}$ & 8 & 81 & 1.093 \\
\hline$U$ & -10.5396 & -1.3122 & 0.802 \\
\hline & -10.4664 & -1.1010 & -0.933 \\
\hline & -11.2429 & 96 & -0.680 \\
\hline$F_{1}$ & -11.3221 & 0851 & 1.065 \\
\hline$F_{1}$ & -13 & -0.6975 & 077 \\
\hline I & -12.9866 & -0.4992 & -0.980 \\
\hline $\mathrm{H}$ & -15.5478 & 2.7979 & -0.764 \\
\hline & -15. & 70 & 0.971 \\
\hline & & & 0.713 \\
\hline $\mathrm{H}$ & -17.5958 & 1.3157 & -1.026 \\
\hline $\mathrm{H}$ & -17.9190 & 3.8131 & -0.824 \\
\hline
\end{tabular}




$\begin{array}{rrrr}\mathrm{H} & -18.0203 & 3.6578 & 0.9226 \\ \mathrm{H} & -20.3116 & 3.8002 & -0.0792 \\ \mathrm{H} & -20.0875 & 2.2139 & 0.6800 \\ \mathrm{H} & -19.9859 & 2.3740 & -1.0806\end{array}$

17chain6

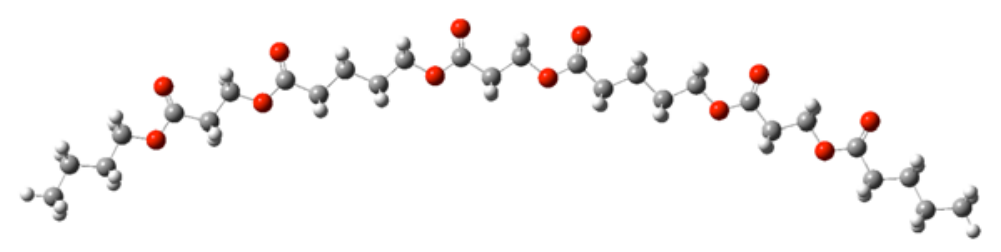

\begin{tabular}{|c|c|c|c|}
\hline & -18 & 47 & 79 \\
\hline & -18.2060 & 2.9584 & -0.1492 \\
\hline & -16.6727 & .0006 & -0.0861 \\
\hline & -16.0577 & .6100 & -0.0498 \\
\hline & -14.6193 & 1.7665 & 0.0076 \\
\hline & -13.9004 & 0.6270 & 0.0329 \\
\hline & -12.4148 & 0.9267 & 0.0935 \\
\hline & -11.5847 & -0.3483 & 0.0774 \\
\hline & -10.2021 & 0.0642 & 0.1378 \\
\hline & -9.2869 & 0.9321 & 991 \\
\hline & -7.8780 & -0.3711 & 1753 \\
\hline & -6.8023 & -1.4516 & 0.0421 \\
\hline & -5.3832 & -0.8749 & 0.1289 \\
\hline & -4.3215 & -1.9560 & -0.0092 \\
\hline & -3.0281 & -1.3117 & 0.0740 \\
\hline & -1.9567 & -2.1 & -0.0268 \\
\hline & -0.6663 & -1.3 & 0.0656 \\
\hline & 0.5527 & -2.2393 & -0.0156 \\
\hline & 1.7093 & -1.3788 & 0.0682 \\
\hline & 2.9104 & -1.9985 & -0.0069 \\
\hline & 4.0426 & -0.9918 & 0.0919 \\
\hline & 5.4235 & -1.6408 & -0.0274 \\
\hline & 6.5609 & -0.6157 & 673 \\
\hline & 7.9279 & -1.2724 & -0.0567 \\
\hline & 8.9253 & -0.2278 & 0.0312 \\
\hline & 10.2093 & -0.6289 & -0.0699 \\
\hline & 11.1551 & 0.5527 & 0.0235 \\
\hline & 12.6097 & 0.1142 & -0.0615 \\
\hline & 13.4043 & 1.3163 & 0.0105 \\
\hline & 14.7473 & 1.1373 & -0.0380 \\
\hline & 15.4716 & 2.4691 & 0.0316 \\
\hline & 16.9951 & 2.3247 & 0.0226 \\
\hline & 17.7217 & 3.6745 & 0.0717 \\
\hline & 19.2477 & 3.5313 & 0.0641 \\
\hline & -14.3858 & -0.4808 & 0.0108 \\
\hline & -9.5755 & -2.1021 & 0.0175 \\
\hline & -2.0282 & -3.3223 & -0.1736 \\
\hline & 3.0390 & -3.1927 & -0.1346 \\
\hline & 10.5466 & -1.7807 & -0.2183 \\
\hline & 15.2692 & 0.0516 & -0.1280 \\
\hline & -19.9312 & 4.2956 & -0.2333 \\
\hline & -18.4962 & 4.9206 & -1.0653 \\
\hline
\end{tabular}




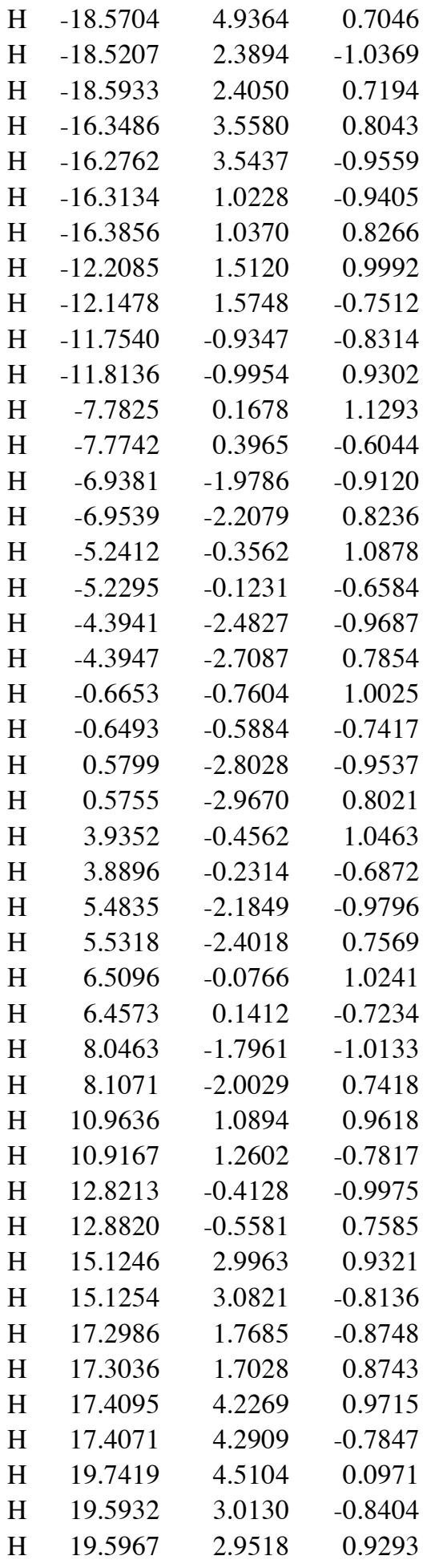

17chain7

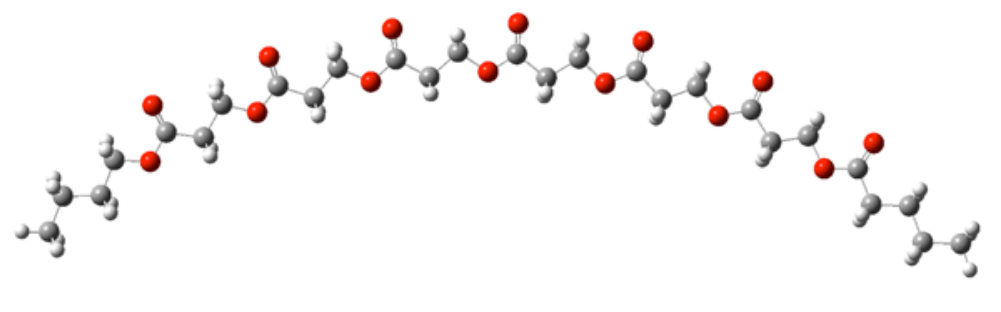

$\begin{array}{llll}\text { C } & 18.1362 & 5.0927 & -0.3997\end{array}$ 


\begin{tabular}{|c|c|c|c|}
\hline & 11 & 3. & \\
\hline & 16.0893 & 3.5730 & -0.2237 \\
\hline & 15.5955 & 2.1442 & -0.0557 \\
\hline & 14.1472 & 2.1716 & 0.0628 \\
\hline & 13.5344 & & 0065 \\
\hline & 12.0259 & 1399 & 0.0466 \\
\hline & 11.3229 & -0.2052 & 149. \\
\hline & 9.9036 & 0711 & 133 \\
\hline & 9.0948 & 1.0075 & 193. \\
\hline 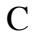 & 7.6366 & 11 & 0.177 \\
\hline$C$ & 6.7133 & 05 & $.181^{\prime}$ \\
\hline & 5.3634 & -1.2 & \\
\hline & 4.3793 & -2.2 & 54. \\
\hline & 3.0163 & & $171 \mathrm{~s}$ \\
\hline C & 1.8954 & -2. & 0.063 \\
\hline C & 0.6580 & -1.8 & 13 \\
\hline $\mathrm{C}$ & -0.4 & -2 & \\
\hline $\mathrm{C}$ & -1.6971 & -1. & 0.065 \\
\hline & -2.9802 & -2. & $-0.121 \delta$ \\
\hline & -4.0658 & -1.5 & -0.013 \\
\hline & -5.3081 & -2.0 & -0.161 \\
\hline$C$ & -6.3 & -0 & \\
\hline $\mathrm{C}$ & -7.76 & & -0.199 \\
\hline & -8.6607 & -0. & \\
\hline & -9.9764 & -0.6 & -0.175 \\
\hline 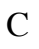 & -10.8121 & 0.6 & 0.015 \\
\hline $\mathrm{C}$ & -12.2 & & -0.150 \\
\hline $\mathrm{O}$ & -12.9 & & 0.062 \\
\hline C & -14.3282 & & -0.052 \\
\hline & -14.9227 & 20 & 0.194 \\
\hline & -16.4492 & & 0.087 \\
\hline C & -17.0412 & 26 & 0.339 \\
\hline C & -18.5 & 1 & .233 \\
\hline $\mathrm{O}$ & 14.1175 & & 80 \\
\hline & 9.4918 & & 0.254 \\
\hline & 4.5726 & -3.2 & 0.121 \\
\hline & -0.4920 & -3.7 & -0.159 \\
\hline $\mathrm{O}$ & -5.5 & -3.1 & 74 \\
\hline $\mathrm{O}$ & -10.4 & -1 & 15 \\
\hline $\mathrm{O}$ & -14.9469 & & -0.316 \\
\hline & 19.2328 & & -0.4002 \\
\hline & 1 & & 13 \\
\hline $\mathrm{H}$ & 17.7891 & 5 & -1.345 \\
\hline $\mathrm{H}$ & 18.0 & 32 & 0.708 \\
\hline $\mathrm{H}$ & 18.0211 & 78 & -1.037 \\
\hline & 15.6869 & & -1.161 \\
\hline & 15.6810 & & 88 \\
\hline $\mathrm{H}$ & 15.9348 & 2 & .888 \\
\hline $\mathrm{H}$ & 15.9 & 6 & -0.867 \\
\hline $\mathrm{H}$ & 11.7341 & 1.6632 & -0.873 \\
\hline $\mathrm{H}$ & 11.7295 & & 0.874 \\
\hline & 11.5 & -0.7 & 1.072 \\
\hline & 11.5768 & -0.8 & 860 \\
\hline & 7.4532 & 0.0363 & -0.704 \\
\hline & 7.4414 & 0.0476 & 1.048 \\
\hline
\end{tabular}




$\begin{array}{rrrr}\mathrm{H} & 6.8677 & -2.4284 & 1.0648 \\ \mathrm{H} & 6.8619 & -2.4326 & -0.6994 \\ \mathrm{H} & 2.9617 & -0.8157 & -0.6488 \\ \mathrm{H} & 2.9196 & -0.9601 & 1.0979 \\ \mathrm{H} & 1.9218 & -3.2884 & 0.8854 \\ \mathrm{H} & 1.9453 & -3.1313 & -0.8717 \\ \mathrm{H} & -1.6125 & -0.8672 & -0.6932 \\ \mathrm{H} & -1.6996 & -1.1411 & 1.0361 \\ \mathrm{H} & -3.0943 & -3.2287 & 0.6400 \\ \mathrm{H} & -3.0183 & -2.9427 & -1.0991 \\ \mathrm{H} & -6.1514 & -0.1375 & -0.7618 \\ \mathrm{H} & -6.2451 & -0.4490 & 0.9606 \\ \mathrm{H} & -7.9960 & -2.2432 & 0.5459 \\ \mathrm{H} & -7.9049 & -1.9252 & -1.1869 \\ \mathrm{H} & -10.4804 & 1.3791 & -0.7021 \\ \mathrm{H} & -10.6064 & 1.0312 & 1.0108 \\ \mathrm{H} & -12.6540 & -0.4093 & 0.5717 \\ \mathrm{H} & -12.5312 & -0.0493 & -1.1503 \\ \mathrm{H} & -14.4623 & 3.6251 & -0.5197 \\ \mathrm{H} & -14.5874 & 3.2654 & 1.1859 \\ \mathrm{H} & -16.8718 & 2.2292 & 0.8006 \\ \mathrm{H} & -16.7465 & 2.5912 & -0.9074 \\ \mathrm{H} & -16.6112 & 5.0588 & -0.3778 \\ \mathrm{H} & -16.7365 & 4.6951 & 1.3368 \\ \mathrm{H} & -18.9660 & 5.3762 & 0.4208 \\ \mathrm{H} & -19.0308 & 3.6891 & 0.9616 \\ \mathrm{H} & -18.9050 & 4.0600 & -0.7652 \\ & & & \end{array}$

18chain0

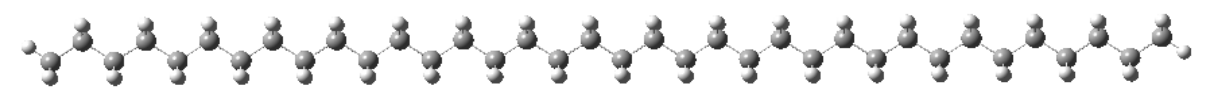

$\begin{array}{lrrr}\mathrm{C} & -0.4192 & 19.9056 & 0.0000 \\ \mathrm{C} & 0.4227 & 18.6227 & 0.0000 \\ \mathrm{C} & -0.4158 & 17.3372 & 0.0000 \\ \mathrm{C} & 0.4258 & 16.0539 & 0.0000 \\ \mathrm{C} & -0.4135 & 14.7689 & 0.0000 \\ \mathrm{C} & 0.4274 & 13.4851 & 0.0000 \\ \mathrm{C} & -0.4125 & 12.2006 & 0.0000 \\ \mathrm{C} & 0.4278 & 10.9163 & 0.0000 \\ \mathrm{C} & -0.4127 & 9.6322 & 0.0000 \\ \mathrm{C} & 0.4271 & 8.3476 & 0.0000 \\ \mathrm{C} & -0.4139 & 7.0638 & 0.0000 \\ \mathrm{C} & 0.4255 & 5.7789 & 0.0000 \\ \mathrm{C} & -0.4158 & 4.4953 & 0.0000 \\ \mathrm{C} & 0.4233 & 3.2103 & 0.0000 \\ \mathrm{C} & -0.4182 & 1.9268 & 0.0000 \\ \mathrm{C} & 0.4208 & 0.6417 & 0.0000 \\ \mathrm{C} & 0.4182 & 21.1916 & 0.0000 \\ \mathrm{C} & -0.4313 & 22.4677 & 0.0000 \\ \mathrm{C} & -0.4208 & -0.6417 & 0.0000 \\ \mathrm{C} & 0.4182 & -1.9268 & 0.0000 \\ \mathrm{C} & -0.4233 & -3.2103 & 0.0000 \\ \mathrm{C} & 0.4158 & -4.4953 & 0.0000\end{array}$




\begin{tabular}{|c|c|c|c|}
\hline 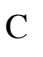 & -0.4255 & -5.7789 & 000 \\
\hline & 0.4139 & -7.0638 & 0.0000 \\
\hline & -0.4271 & -8.3476 & 0.0000 \\
\hline & 0.4127 & -9.6322 & 0000 \\
\hline C & -0.4278 & -10.9163 & .0000 \\
\hline $\mathrm{C}$ & 0.4125 & -12.2006 & 0.0000 \\
\hline ( & -0.4274 & -13.4851 & 0.0000 \\
\hline & 0.4135 & -14.7689 & 0.0000 \\
\hline & -0.4258 & -16.0539 & 0.0000 \\
\hline$C$ & 0.4158 & -17.3372 & 0.0000 \\
\hline $\mathrm{C}$ & -0.4227 & -18.6227 & 0.0000 \\
\hline $\mathrm{C}$ & 0.4192 & -19.9056 & 0.0000 \\
\hline c & -0.4182 & -21.1916 & 0.0000 \\
\hline c & 0.4313 & -22.4677 & 0.0000 \\
\hline $\mathrm{H}$ & -1.0838 & 19. & .8788 \\
\hline $\mathrm{H}$ & -1.0838 & 19.9055 & -0.8788 \\
\hline $\mathrm{H}$ & 1.0870 & 18.6236 & 0.8788 \\
\hline I & 1.0870 & 18.6236 & -0.8788 \\
\hline & -1.0802 & 17.3365 & -0.8787 \\
\hline & -1.0802 & 17. & 0.8787 \\
\hline $\mathrm{H}$ & 1.0901 & 16. & 0.8787 \\
\hline F & 1.0901 & 16.0545 & -0.8787 \\
\hline $\mathrm{H}$ & -1.0778 & 14.7685 & -0.8787 \\
\hline & -1.0778 & 14.7685 & 0.8787 \\
\hline & 1.0917 & 13.4853 & 0.8787 \\
\hline $\mathrm{H}$ & 1.0917 & 13. & -0.8787 \\
\hline $\mathrm{H}$ & -1.0769 & 005 & -0.8787 \\
\hline $\mathrm{H}$ & -1.0769 & 12.2005 & 0.8787 \\
\hline $\mathrm{H}$ & 1.0921 & 10.9163 & 0.8787 \\
\hline & 1.0921 & 10.9163 & -0.8787 \\
\hline $\mathrm{H}$ & -1.0771 & 24 & -0.8787 \\
\hline $\mathrm{H}$ & -1.0771 & 24 & 0.8787 \\
\hline $\mathrm{H}$ & 1.0914 & 8.3473 & 0.8787 \\
\hline $\mathrm{H}$ & 1.0914 & 73 & -0.8787 \\
\hline $\mathrm{H}$ & -1.0782 & 7.0642 & -0.8787 \\
\hline $\mathrm{H}$ & -1.0782 & 7.0642 & 0.8787 \\
\hline $\mathrm{H}$ & 1.0898 & 5.7784 & 0.8787 \\
\hline $\mathrm{H}$ & 1.0898 & 784 & -0.8787 \\
\hline $\mathrm{H}$ & -1.0802 & 4.4959 & -0.8787 \\
\hline $\mathrm{H}$ & -1.0802 & 4.4959 & 0.8787 \\
\hline $\mathrm{H}$ & 1.0876 & 3.2097 & 0.8787 \\
\hline $\mathrm{H}$ & 1.0876 & 3.2097 & -0.8787 \\
\hline $\mathrm{H}$ & -1.0825 & 1.9275 & -0.8787 \\
\hline $\mathrm{H}$ & -1.0825 & 1.9275 & 0.8787 \\
\hline $\mathrm{H}$ & 1.0851 & 0.6410 & 0.8787 \\
\hline $\mathrm{H}$ & 1.0851 & 0.6410 & -0.8787 \\
\hline $\mathrm{H}$ & 1.0815 & 21.1923 & -0.8785 \\
\hline $\mathrm{H}$ & 1.0815 & 21.1923 & 0.8785 \\
\hline $\mathrm{H}$ & 0.1957 & 23.3686 & 0.0000 \\
\hline $\mathrm{H}$ & -1.0799 & 22.5134 & 0.8854 \\
\hline $\mathrm{H}$ & -1.0799 & 22.5134 & -0.8854 \\
\hline $\mathrm{H}$ & -1.0851 & -0.6410 & -0.8787 \\
\hline $\mathrm{H}$ & -1.0851 & -0.6410 & 0.8787 \\
\hline $\mathrm{H}$ & 1.0825 & -1.9275 & 0.8787 \\
\hline $\mathrm{H}$ & 1.0825 & -1.9275 & -0.8787 \\
\hline & & & \\
\hline
\end{tabular}




\begin{tabular}{lrrr}
$\mathrm{H}$ & -1.0876 & -3.2097 & -0.8787 \\
$\mathrm{H}$ & -1.0876 & -3.2097 & 0.8787 \\
$\mathrm{H}$ & 1.0802 & -4.4959 & 0.8787 \\
$\mathrm{H}$ & 1.0802 & -4.4959 & -0.8787 \\
$\mathrm{H}$ & -1.0898 & -5.7784 & -0.8787 \\
$\mathrm{H}$ & -1.0898 & -5.7784 & 0.8787 \\
$\mathrm{H}$ & 1.0782 & -7.0642 & 0.8787 \\
$\mathrm{H}$ & 1.0782 & -7.0642 & -0.8787 \\
$\mathrm{H}$ & -1.0914 & -8.3473 & -0.8787 \\
$\mathrm{H}$ & -1.0914 & -8.3473 & 0.8787 \\
$\mathrm{H}$ & 1.0771 & -9.6324 & 0.8787 \\
$\mathrm{H}$ & 1.0771 & -9.6324 & -0.8787 \\
$\mathrm{H}$ & -1.0921 & -10.9163 & -0.8787 \\
$\mathrm{H}$ & -1.0921 & -10.9163 & 0.8787 \\
$\mathrm{H}$ & 1.0769 & -12.2005 & 0.8787 \\
$\mathrm{H}$ & 1.0769 & -12.2005 & -0.8787 \\
$\mathrm{H}$ & -1.0917 & -13.4853 & -0.8787 \\
$\mathrm{H}$ & -1.0917 & -13.4853 & 0.8787 \\
$\mathrm{H}$ & 1.0778 & -14.7685 & 0.8787 \\
$\mathrm{H}$ & 1.0778 & -14.7685 & -0.8787 \\
$\mathrm{H}$ & -1.0901 & -16.0545 & -0.8787 \\
$\mathrm{H}$ & -1.0901 & -16.0545 & 0.8787 \\
$\mathrm{H}$ & 1.0802 & -17.3365 & 0.8787 \\
$\mathrm{H}$ & 1.0802 & -17.3365 & -0.8787 \\
$\mathrm{H}$ & -1.0870 & -18.6236 & -0.8788 \\
$\mathrm{H}$ & -1.0870 & -18.6236 & 0.8788 \\
$\mathrm{H}$ & 1.0838 & -19.9055 & 0.8788 \\
$\mathrm{H}$ & 1.0838 & -19.9055 & -0.8788 \\
$\mathrm{H}$ & -1.0815 & -21.1923 & -0.8785 \\
$\mathrm{H}$ & -1.0815 & -21.1923 & 0.8785 \\
$\mathrm{H}$ & -0.1957 & -23.3686 & 0.0000 \\
$\mathrm{H}$ & 1.0799 & -22.5134 & 0.8854 \\
$\mathrm{H}$ & 1.0799 & -22.5134 & -0.8854 \\
& & & \\
\hline
\end{tabular}

19chain0

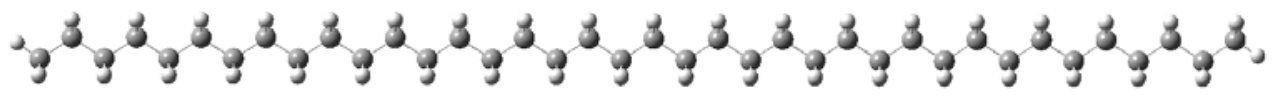

$\begin{array}{rrrr}\text { C } & 23.7522 & 0.3947 & -0.0001 \\ \text { C } & 22.4748 & -0.4526 & -0.0001 \\ \text { C } & 21.1902 & 0.3870 & 0.0000 \\ \text { C } & 19.9059 & -0.4529 & 0.0000 \\ \text { C } & 18.6218 & 0.3876 & 0.0001 \\ \text { C } & 17.3372 & -0.4520 & 0.0001 \\ \text { C } & 16.0536 & 0.3892 & 0.0002 \\ \text { C } & 14.7685 & -0.4498 & 0.0003 \\ \text { C } & 13.4853 & 0.3921 & 0.0003 \\ \text { C } & 12.1998 & -0.4464 & 0.0004 \\ \text { C } & 10.9169 & 0.3960 & 0.0004 \\ \text { C } & 9.6311 & -0.4420 & 0.0005 \\ \text { C } & 8.3485 & 0.4008 & 0.0005 \\ \text { C } & 7.0625 & -0.4367 & 0.0005 \\ \text { C } & 5.7801 & 0.4064 & 0.0005 \\ \text { C } & 4.4939 & -0.4309 & 0.0005 \\ & & \text { S52 } & \end{array}$




\begin{tabular}{|c|c|c|c|}
\hline & 3.2117 & & \\
\hline & 1.9253 & -0.4246 & 0.000 \\
\hline & 0.6432 & 0.4188 & 0.0004 \\
\hline & -0.6432 & -0.4183 & 0.0004 \\
\hline & -1.9253 & .42 & 0.000 \\
\hline & -3.2117 & -0.4119 & 0.000 \\
\hline & -4.4939 & 0.4314 & 0.000 \\
\hline & -5.7801 & -0.4059 & 0.000 \\
\hline & -7.0625 & 0.4371 & 0.000 \\
\hline & -8.3485 & -0.4005 & -0.0001 \\
\hline & -9.6312 & 0.4422 & 0.000 \\
\hline C & -10.9169 & -0.3 & 0.000 \\
\hline & -12.1998 & 0.4465 & -0.0003 \\
\hline & -13.4852 & -0.3920 & -0.000 \\
\hline & -14.7685 & 0.4497 & -0.000 \\
\hline & -16.0535 & -0.3894 & -0.000 \\
\hline C & -17.3372 & 0.4 & -0.000 \\
\hline & -18.6218 & -0.3880 & $-0.000^{\circ}$ \\
\hline & -19.9059 & 0.4524 & $-0.000^{\prime}$ \\
\hline & -21.1901 & -0.3876 & -0.0008 \\
\hline & -22.4748 & 0.4519 & -0.000 \\
\hline C & -23.7522 & -0.3 & -0.000 \\
\hline & 24.6520 & -0.2 & -0.000 \\
\hline & 23.7992 & 32 & 0.885 \\
\hline & 23.7991 & 1.0433 & -0.885 \\
\hline & 22.4745 & -1.1159 & 0.878 \\
\hline & 22.4744 & -1 . & $-0.878 c$ \\
\hline $\mathrm{H}$ & 21.1 & & -0.878 \\
\hline $\mathrm{H}$ & 21.1 & 14 & 0.878 \\
\hline & 19.9058 & 73 & 0.878 \\
\hline & 19.9058 & -1.1 & $-0.878 \varepsilon$ \\
\hline & 18.6221 & 1.0520 & $-0.878 c$ \\
\hline H & 18.62 & 19 & 0.878 \\
\hline $\mathrm{H}$ & 17.3 & 64 & .878 \\
\hline & 17.3367 & -1 . & $-0.878 \mathrm{C}$ \\
\hline & 16.0541 & 36 & -0.878 \\
\hline & 16.0542 & 05 & 0.879 \\
\hline $\mathrm{H}$ & 14.7678 & -1 . & 0.879 \\
\hline $\mathrm{H}$ & 14.7 & -1 . & -0.878 \\
\hline $\mathrm{H}$ & 13.4861 & & -0.878 \\
\hline & 13.4 & & 0.879 \\
\hline & 12.1988 & -1.1 & 0.879 \\
\hline & 12.1988 & -1 & -0.878 \\
\hline $\mathrm{H}$ & 10.9180 & & -0.878 \\
\hline $\mathrm{H}$ & 10.9181 & 03 & 0.879 \\
\hline & 9.6299 & -1.1063 & 0.879 \\
\hline & 9.6299 & -1.1 & -0.878 \\
\hline & 8.3499 & 51 & -0.878 \\
\hline $\mathrm{H}$ & 8.3499 & 52 & 0.879 \\
\hline $\mathrm{H}$ & 7.0611 & -1.1010 & 0.879 \\
\hline 11 & 7.0611 & -1.1011 & -0.8782 \\
\hline 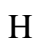 & 5.7816 & 1.0707 & -0.878 \\
\hline & 5.7816 & 708 & 0.879 \\
\hline & 4.4923 & -1.0952 & 0.879 \\
\hline $\mathrm{H}$ & 4.4924 & -1.0953 & -0.878 \\
\hline
\end{tabular}




\begin{tabular}{|c|c|c|c|}
\hline $\mathrm{H}$ & 3.2133 & 1.0767 & -0.8783 \\
\hline $\mathrm{H}$ & 3.2133 & 1.0769 & 0.8792 \\
\hline $\mathrm{H}$ & 1.9237 & -1.0889 & 0.8793 \\
\hline $\mathrm{H}$ & 1.9237 & -1.0891 & -0.8782 \\
\hline $\mathrm{H}$ & 0.6449 & 1.0831 & -0.8784 \\
\hline $\mathrm{H}$ & 0.6448 & 1.0832 & 0.8791 \\
\hline $\mathrm{H}$ & -0.6449 & -1.0825 & 0.8792 \\
\hline $\mathrm{H}$ & -0.6448 & -1.0827 & -0.8783 \\
\hline $\mathrm{H}$ & -1.9237 & 1.0895 & -0.8785 \\
\hline $\mathrm{H}$ & -1.9238 & 1.0896 & 0.8790 \\
\hline $\mathrm{H}$ & -3.2133 & -1.0762 & 0.8790 \\
\hline $\mathrm{H}$ & -3.2132 & -1.0763 & -0.8785 \\
\hline $\mathrm{H}$ & -4.4923 & 1.0957 & -0.8787 \\
\hline $\mathrm{H}$ & -4.4924 & 1.0957 & 0.8788 \\
\hline $\mathrm{H}$ & -5.7816 & -1.0703 & 0.8788 \\
\hline $\mathrm{H}$ & -5.7815 & -1.0703 & -0.8786 \\
\hline $\mathrm{H}$ & -7.0611 & 1.1014 & -0.8788 \\
\hline $\mathrm{H}$ & -7.0612 & 1.1015 & 0.8787 \\
\hline $\mathrm{H}$ & -8.3499 & -1.0648 & 0.8786 \\
\hline $\mathrm{H}$ & -8.3498 & -1.0649 & -0.8788 \\
\hline $\mathrm{H}$ & -9.6299 & 1.1065 & -0.8789 \\
\hline $\mathrm{H}$ & -9.6300 & 1.1066 & 0.8785 \\
\hline $\mathrm{H}$ & -10.9180 & -1.0601 & 0.8785 \\
\hline $\mathrm{H}$ & -10.9179 & -1.0601 & -0.8790 \\
\hline $\mathrm{H}$ & -12.1988 & 1.1108 & -0.8790 \\
\hline $\mathrm{H}$ & -12.1989 & 1.1108 & 0.8784 \\
\hline $\mathrm{H}$ & -13.4861 & -1.0564 & 0.8783 \\
\hline $\mathrm{H}$ & -13.4860 & -1.0564 & -0.8791 \\
\hline $\mathrm{H}$ & -14.7678 & 1.1140 & -0.8791 \\
\hline $\mathrm{H}$ & -14.7679 & 1.1140 & 0.8783 \\
\hline $\mathrm{H}$ & -16.0541 & -1.0538 & 0.8782 \\
\hline $\mathrm{H}$ & -16.0540 & -1.0537 & -0.8793 \\
\hline $\mathrm{H}$ & -17.3368 & 1.1161 & -0.8793 \\
\hline $\mathrm{H}$ & -17.3369 & 1.1160 & 0.8782 \\
\hline $\mathrm{H}$ & -18.6221 & -1.0524 & 0.8780 \\
\hline $\mathrm{H}$ & -18.6220 & -1.0523 & -0.8794 \\
\hline $\mathrm{H}$ & -19.9059 & 1.1168 & -0.8794 \\
\hline $\mathrm{H}$ & -19.9059 & 1.1167 & 0.8781 \\
\hline $\mathrm{H}$ & -21.1911 & -1.0521 & 0.8780 \\
\hline $\mathrm{H}$ & -21.1910 & -1.0521 & -0.8796 \\
\hline $\mathrm{H}$ & -22.4745 & 1.1152 & -0.8793 \\
\hline $\mathrm{H}$ & -22.4746 & 1.1151 & 0.8777 \\
\hline $\mathrm{H}$ & -24.6521 & 0.2329 & -0.0009 \\
\hline $\mathrm{H}$ & -23.7991 & -1.0441 & 0.8845 \\
\hline $\mathrm{H}$ & -23.7990 & -1.0440 & -0.8864 \\
\hline
\end{tabular}

19chain1

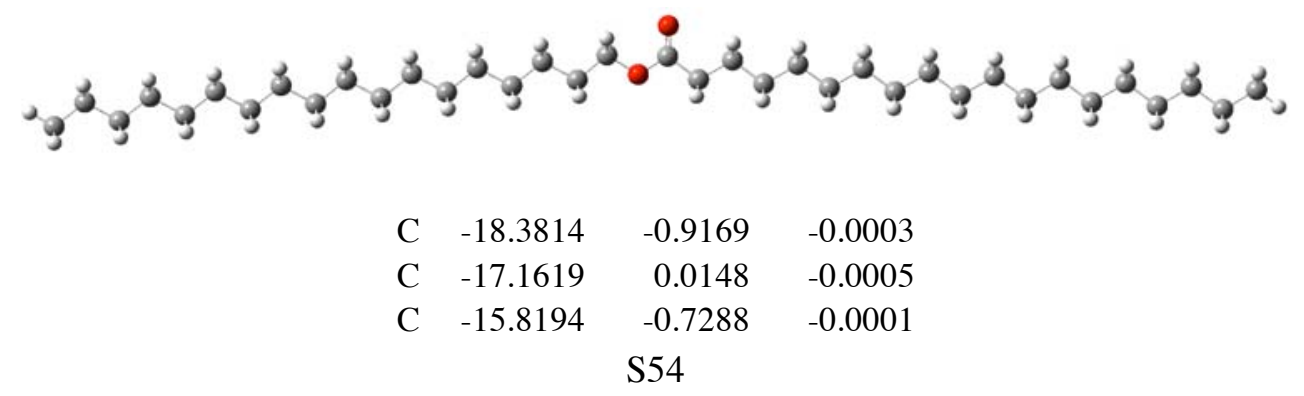




\begin{tabular}{|c|c|c|c|}
\hline $\mathrm{C}$ & -14.6010 & 0.2042 & -0.0003 \\
\hline $\mathrm{C}$ & -13.2575 & -0.5377 & 0.0001 \\
\hline $\mathrm{C}$ & -12.0406 & 0.3973 & 0.0000 \\
\hline $\mathrm{C}$ & -10.6958 & -0.3422 & 0.0004 \\
\hline $\mathrm{C}$ & -9.4810 & 0.5956 & 0.0004 \\
\hline $\mathrm{C}$ & -8.1344 & -0.1407 & 0.0007 \\
\hline $\mathrm{C}$ & -6.9226 & 0.8009 & 0.0006 \\
\hline $\mathrm{C}$ & -5.5743 & 0.0681 & 0.0009 \\
\hline C & -4.3668 & 1.0154 & 0.0007 \\
\hline $\mathrm{C}$ & -3.0194 & 0.2802 & 0.0009 \\
\hline C & -1.8411 & 1.2429 & 0.0007 \\
\hline $\mathrm{O}$ & -0.6267 & 0.4592 & 0.0008 \\
\hline $\mathrm{C}$ & 0.5294 & 1.1616 & -0.0002 \\
\hline $\mathrm{C}$ & 1.7285 & 0.2305 & -0.0002 \\
\hline $\mathrm{C}$ & 3.0660 & 0.9746 & -0.0002 \\
\hline $\mathrm{C}$ & 4.2746 & 0.0301 & -0.0001 \\
\hline $\mathrm{C}$ & 5.6187 & 0.7709 & -0.0001 \\
\hline $\mathrm{C}$ & 6.8364 & -0.1629 & -0.0001 \\
\hline $\mathrm{C}$ & 8.1782 & 0.5821 & -0.0002 \\
\hline $\mathrm{C}$ & 9.3991 & -0.3476 & -0.0002 \\
\hline $\mathrm{C}$ & 10.7391 & 0.4006 & -0.0003 \\
\hline $\mathrm{C}$ & 11.9620 & -0.5265 & -0.0003 \\
\hline $\mathrm{C}$ & 13.3008 & 0.2239 & -0.0004 \\
\hline $\mathrm{C}$ & 14.5250 & -0.7016 & -0.0003 \\
\hline $\mathrm{C}$ & 15.8630 & 0.0502 & -0.0004 \\
\hline $\mathrm{C}$ & 17.0880 & -0.8742 & -0.0002 \\
\hline $\mathrm{C}$ & 18.4256 & -0.1218 & -0.0002 \\
\hline $\mathrm{O}$ & 0.5752 & 2.3701 & -0.0011 \\
\hline $\mathrm{C}$ & -19.7233 & -0.1721 & -0.0006 \\
\hline $\mathrm{C}$ & -20.9434 & -1.1027 & -0.0002 \\
\hline $\mathrm{C}$ & 19.6508 & -1.0459 & 0.0000 \\
\hline $\mathrm{C}$ & 20.9884 & -0.2939 & 0.0001 \\
\hline $\mathrm{C}$ & -22.2852 & -0.3580 & -0.0005 \\
\hline $\mathrm{C}$ & -23.4984 & -1.2948 & -0.0001 \\
\hline $\mathrm{C}$ & 22.2135 & -1.2180 & 0.0004 \\
\hline $\mathrm{C}$ & 23.5455 & -0.4593 & 0.0004 \\
\hline $\mathrm{H}$ & -18.3332 & -1.5792 & 0.8787 \\
\hline $\mathrm{H}$ & -18.3331 & -1.5797 & -0.8788 \\
\hline $\mathrm{H}$ & -17.2105 & 0.6778 & 0.8780 \\
\hline $\mathrm{H}$ & -17.2104 & 0.6772 & -0.8794 \\
\hline $\mathrm{H}$ & -15.7705 & -1.3916 & -0.8787 \\
\hline $\mathrm{H}$ & -15.7707 & -1.3911 & 0.8788 \\
\hline $\mathrm{H}$ & -14.6504 & 0.8670 & 0.8782 \\
\hline $\mathrm{H}$ & -14.6502 & 0.8665 & -0.8792 \\
\hline $\mathrm{H}$ & -13.2075 & -1.2004 & -0.8785 \\
\hline $\mathrm{H}$ & -13.2078 & -1.1999 & 0.8791 \\
\hline $\mathrm{H}$ & -12.0911 & 1.0600 & 0.8785 \\
\hline $\mathrm{H}$ & -12.0909 & 1.0595 & -0.8789 \\
\hline $\mathrm{H}$ & -10.6444 & -1.0047 & -0.8783 \\
\hline $\mathrm{H}$ & -10.6447 & -1.0043 & 0.8793 \\
\hline $\mathrm{H}$ & -9.5330 & 1.2581 & 0.8789 \\
\hline $\mathrm{H}$ & -9.5328 & 1.2577 & -0.8785 \\
\hline $\mathrm{H}$ & -8.0812 & -0.8029 & -0.8781 \\
\hline $\mathrm{H}$ & -8.0814 & -0.8026 & 0.8796 \\
\hline $\mathrm{H}$ & -6.9764 & 1.4630 & 0.8793 \\
\hline \multicolumn{4}{|c|}{ S55 } \\
\hline
\end{tabular}




\begin{tabular}{|c|c|c|c|}
\hline $\mathrm{H}$ & -6.9763 & 1.4628 & -0.8782 \\
\hline $\mathrm{H}$ & -5.5179 & -0.5933 & -0.8779 \\
\hline $\mathrm{H}$ & -5.5180 & -0.5929 & 0.8800 \\
\hline $\mathrm{H}$ & -4.4239 & 1.6766 & 0.8796 \\
\hline $\mathrm{H}$ & -4.4239 & 1.6762 & -0.8784 \\
\hline $\mathrm{H}$ & -2.9468 & -0.3738 & -0.8797 \\
\hline $\mathrm{H}$ & -2.9468 & -0.3734 & 0.8819 \\
\hline $\mathrm{H}$ & -1.8438 & 1.8937 & 0.8843 \\
\hline $\mathrm{H}$ & -1.8439 & 1.8934 & -0.8831 \\
\hline $\mathrm{H}$ & 1.6402 & -0.4315 & -0.8740 \\
\hline $\mathrm{H}$ & 1.6402 & -0.4316 & 0.8735 \\
\hline $\mathrm{H}$ & 3.1084 & 1.6376 & 0.8747 \\
\hline $\mathrm{H}$ & 3.1085 & 1.6375 & -0.8752 \\
\hline $\mathrm{H}$ & 4.2242 & -0.6325 & -0.8789 \\
\hline $\mathrm{H}$ & 4.2241 & -0.6323 & 0.8788 \\
\hline $\mathrm{H}$ & 5.6674 & 1.4334 & 0.8784 \\
\hline $\mathrm{H}$ & 5.6674 & 1.4333 & -0.8786 \\
\hline $\mathrm{H}$ & 6.7878 & -0.8253 & -0.8789 \\
\hline $\mathrm{H}$ & 6.7878 & -0.8252 & 0.8788 \\
\hline $\mathrm{H}$ & 8.2257 & 1.2449 & 0.8784 \\
\hline $\mathrm{H}$ & 8.2256 & 1.2448 & -0.8789 \\
\hline $\mathrm{H}$ & 9.3522 & -1.0103 & -0.8789 \\
\hline $\mathrm{H}$ & 9.3523 & -1.0102 & 0.8787 \\
\hline $\mathrm{H}$ & 10.7852 & 1.0635 & 0.8783 \\
\hline $\mathrm{H}$ & 10.7852 & 1.0633 & -0.8791 \\
\hline $\mathrm{H}$ & 11.9164 & -1.1894 & -0.8790 \\
\hline $\mathrm{H}$ & 11.9165 & -1.1892 & 0.8785 \\
\hline $\mathrm{H}$ & 13.3460 & 0.8868 & 0.8782 \\
\hline $\mathrm{H}$ & 13.3459 & 0.8866 & -0.8792 \\
\hline $\mathrm{H}$ & 14.4803 & -1.3645 & -0.8790 \\
\hline $\mathrm{H}$ & 14.4803 & -1.3643 & 0.8785 \\
\hline $\mathrm{H}$ & 15.9076 & 0.7133 & 0.8782 \\
\hline $\mathrm{H}$ & 15.9076 & 0.7130 & -0.8792 \\
\hline $\mathrm{H}$ & 17.0437 & -1.5372 & -0.8789 \\
\hline $\mathrm{H}$ & 17.0436 & -1.5370 & 0.8786 \\
\hline $\mathrm{H}$ & 18.4698 & 0.5412 & 0.8784 \\
\hline $\mathrm{H}$ & 18.4700 & 0.5410 & -0.8791 \\
\hline $\mathrm{H}$ & -19.7713 & 0.4902 & -0.8795 \\
\hline $\mathrm{H}$ & -19.7713 & 0.4908 & 0.8780 \\
\hline $\mathrm{H}$ & -20.8965 & -1.7653 & 0.8788 \\
\hline $\mathrm{H}$ & -20.8965 & -1.7659 & -0.8788 \\
\hline $\mathrm{H}$ & 19.6065 & -1.7090 & -0.8786 \\
\hline $\mathrm{H}$ & 19.6063 & -1.7087 & 0.8789 \\
\hline $\mathrm{H}$ & 21.0338 & 0.3692 & 0.8787 \\
\hline $\mathrm{H}$ & 21.0340 & 0.3689 & -0.8788 \\
\hline $\mathrm{H}$ & -22.3325 & 0.3034 & -0.8792 \\
\hline $\mathrm{H}$ & -22.3325 & 0.3040 & 0.8778 \\
\hline $\mathrm{H}$ & -24.4410 & -0.7326 & -0.0003 \\
\hline $\mathrm{H}$ & -23.4987 & -1.9448 & 0.8855 \\
\hline $\mathrm{H}$ & -23.4987 & -1.9454 & -0.8853 \\
\hline $\mathrm{H}$ & 22.1687 & -1.8799 & -0.8780 \\
\hline $\mathrm{H}$ & 22.1685 & -1.8797 & 0.8789 \\
\hline $\mathrm{H}$ & 24.4004 & -1.1476 & 0.0006 \\
\hline $\mathrm{H}$ & 23.6366 & 0.1846 & 0.8858 \\
\hline $\mathrm{H}$ & 23.6368 & 0.1844 & -0.8851 \\
\hline
\end{tabular}


1) Britovsek, G. J. P.; Gibson, V. C.; Kimberley, B. S.; Maddox, P. J.; McTavish, S. J.; Solan, G. A.; White, A. J. P.; Williams, D. J. Chem. Commun. 1998, 849-850.

2) Britovsek, G. J. P.; Bruce, M.; Gibson, V. C.; Kimberley, B. S.; Maddox, P. J.; Mastroianni, S.; McTavish, S. J.; Redshaw, C.; Solan, G. A.; Strömberg, S.; White, A. J. P.; Williams, D. J. J. Am. Chem. Soc. 1999, 121, 8728-8740.

3) Small, B. L. Organometallics 2003, 22, 3178-3183.

4) Small, B. L.; Brookhart, M. J. Am. Chem. Soc. 1998, 120, 7143-7144.

5) (a) Canich, J. A. M.; Vaughan, G. A.; Matsunaga, P. T.; Gindelberger, D. E.; Shaffer, T. D.; Squire, K. R. PCT Int. Appl. 1997, WO 9748735. (b) Shao, Q.; Sun, H.; Shen, Q.; Zhang, Y. Appl. Organometal. Chem. 2004, 18, 289-290.

6) (a) Gonsalvi, L.; Gaunt, J. A.; Adams, H.; Castro, A.; Sunley, G. J.; Haynes, A. Organometallics 2003, 22, 1047-1054. (b) Pesch, J.; Harms, K.; Bach, T. Eur. J. Org. Chem. 2004, 2025-2035.

7) Frisch, M. J.; Trucks, G. W.; Schlegel, H. B.; Scuseria, G. E.; Robb, M. A.; Cheeseman, J. R.; Montgomery, J. A., Jr.; Vreven, T.; Kudin, K. N.; Burant, J. C.; Millam, J. M.; Iyengar, S. S.; Tomasi, J.; Barone, V.; Mennucci, B.; Cossi, M.; Scalmani, G.; Rega, N.; Petersson, G. A.; Nakatsuji, H.; Hada, M.; Ehara, M.; Toyota, K.; Fukuda, R.; Hasegawa, J.; Ishida, M.; Nakajima, T.; Honda, Y.; Kitao, O.; Nakai, H.; Klene, M.; Li, X.; Knox, J. E.; Hratchian, H. P.; Cross, J. B.; Bakken, V.; Adamo, C.; Jaramillo, J.; Gomperts, R.; Stratmann, R. E.; Yazyev, O.; Austin, A. J.; Cammi, R.; Pomelli, C.; Ochterski, J. W.; Ayala, P. Y.; Morokuma, K.; Voth, G. A.; Salvador, P.; Dannenberg, J. J.; Zakrzewski, V. G.; Dapprich, S.; Daniels, A. D.; Strain, M. C.; Farkas, O.; Malick, D. K.; Rabuck, A. D.; Raghavachari, K.; Foresman, J. B.; Ortiz, J. V.; Cui, Q.; Baboul, A. G.; Clifford, S.; Cioslowski, J.; Stefanov, B. B.; Liu, G.; Liashenko, A.; Piskorz, P.; Komaromi, I.; Martin, R. L.; Fox, D. J.; Keith, T.; Al-Laham, M. A.; Peng, C. Y.; Nanayakkara, A.; Challacombe, M.; Gill, P. M. W.; Johnson, B.; Chen, W.; Wong, M. W.; Gonzalez, C.; Pople, J. A. Gaussian 03, revision C.02; Gaussian, Inc.: Wallingford, CT, 2004.

8) Becke, A. D. J. Chem. Phys. 1993, 98, 5648-5652.

9) Lee, C.; Yang, W.; Parr, R. G. Phys. Rev. 1988, B37, 785-789.

10) Miehlich, B.; Savin, A.; Stoll, H.; Preuss, H. Chem. Phys. Lett. 1989, 157, 200-206. 